\title{
Palladium-Catalyzed Enantioselective Intermolecular Carboetherification of Dihydrofurans
}

\author{
Gustavo M. Borrajo-Calleja, Vincent Bizet, Clément Mazet* \\ Department of Organic Chemistry, University of Geneva \\ Quai Ernest Ansermet 30, 1211 Geneva 4, Switzerland \\ email: clement.mazet@unige.ch
}

\section{Supporting Information}

\section{Table of Contents}

1 General $\quad$ S2

2 Optimization of the intermolecular carboetherification of 2,3-dihydrofuran $\quad$ S3

2.1 Optimization of the carboetherification of 2,3-dhf with 2-bromophenol (racemic version) S3

2.2 Optimization of the carboetherification of 2,3-dhf with 2-bromophenol and a $\mathrm{Pd}^{0}$ source (enantioselective version)

2.3 Optimization of the carboetherification of 2,3-dhf with 2-bromophenol and a Pd" source (enantioselective version)

3 Experimental procedures

3.1 General procedure for the racemic intermolecular carboetherification of 2,3-dihydrofuran using 2-bromophenol derivatives (GP1)

3.2 General procedure for the enantioselective intermolecular carboetherification of 2,3dihydrofuran using 2-bromophenol derivatives (GP2) $\quad$ S8

3.3 Synthesis and characterization of rac-2-(p-tolyl)-2,3-dihydrofuran (1c) S9

3.4 Synthesis and characterization of trans-rac-3-methyl-2-(p-tolyl)-2,3-dihydrofuran (1d) S10

4 Characterization data $\quad$ S11

$5 \quad$ NMR spectra $\quad$ S29

$\begin{array}{lll}6 & \text { X-ray structure analyses } & 574\end{array}$ 


\section{General}

All reactions were carried out under an inert atmosphere of nitrogen using either two-manifold vacuum/inert gas lines or a $M$. Braun glove-box, unless otherwise noted. Solvents were dried over activated alumina columns and further degassed by three successive "freeze-pump-thaw" cycles if necessary.

Unless otherwise noted, commercial reagents were purchased from Aldrich, Acros or Strem and used without further purification. Liquid reagents were transferred with stainless steel syringes or cannula. Thin layer chromatography (TLC) was performed on plates of silica pre-coated with 0.25 mm Kieselgel $60 \mathrm{~F}_{254}$ from Merck. Flash chromatography was performed using silica gel SiliaFlash ${ }^{\circledR}$ P60 (230-400 mesh) from Silicycle. NMR spectra were recorded on ARX-300 and AMX-400 and AM500 Bruker Advance spectrometers. ${ }^{1} \mathrm{H}$ and ${ }^{13} \mathrm{C}\left\{{ }^{1} \mathrm{H}\right\}$ NMR chemical shifts are given in ppm relative to $\mathrm{SiMe}_{4}$, with the solvent resonance used as internal reference. ${ }^{19} \mathrm{~F}\left\{{ }^{1} \mathrm{H}\right\}$ NMR chemical shifts are reported in ppm relative to $\mathrm{CFCl}_{3}$. Infrared spectra were obtained on a Perkin-Elmer $1650 \mathrm{FT}-\mathrm{IR}$ spectrometer using neat samples on a diamond ATR Golden Gate sampler. GC-MS analyses were performed on GC - HP 6890, column Agilent - HP1 (30 m - ID 0.32 mm, Film $0.25 \mu \mathrm{m}$ ) coupled with MS - HP 5973. The enantiomeric excesses (ee's) were determined by HPLC or GC analyses. HPLC analyses were performed on Shimadzu CTO-20AA, with column DAICEL OD-H, OJ-H, AD-H and IC. GC analyses were performed on HP - 6890, column Lipodex $E, 50 \mathrm{~m}$. Retention times $\left(t_{R}\right)$ are given in minutes.

Commercially available 2,3-dihydrofuran and 5-Methyl-2,3-dihydrofurn were distilled over sodium and stored in a Young valve Schlenk under a $\mathrm{N}_{2}$ atmosphere in a refrigerator. To remove possible traces of water, the 2-bromophenol derivatives were dried under $\mathrm{P}_{2} \mathrm{O}_{5}$. Deionized water was degassed and stored in a Young valve Schlenk under a $\mathrm{N}_{2}$ atmosphere. 2-bromo-4-methoxyphenol was prepared following a previously reported procedure. ${ }^{1}$ 


\section{Optimization of the intermolecular carboetherification of 2,3-dihydrofuran}

\subsection{Optimization of the carboetherification of 2,3-dhf with 2-bromophenol (racemic version)}

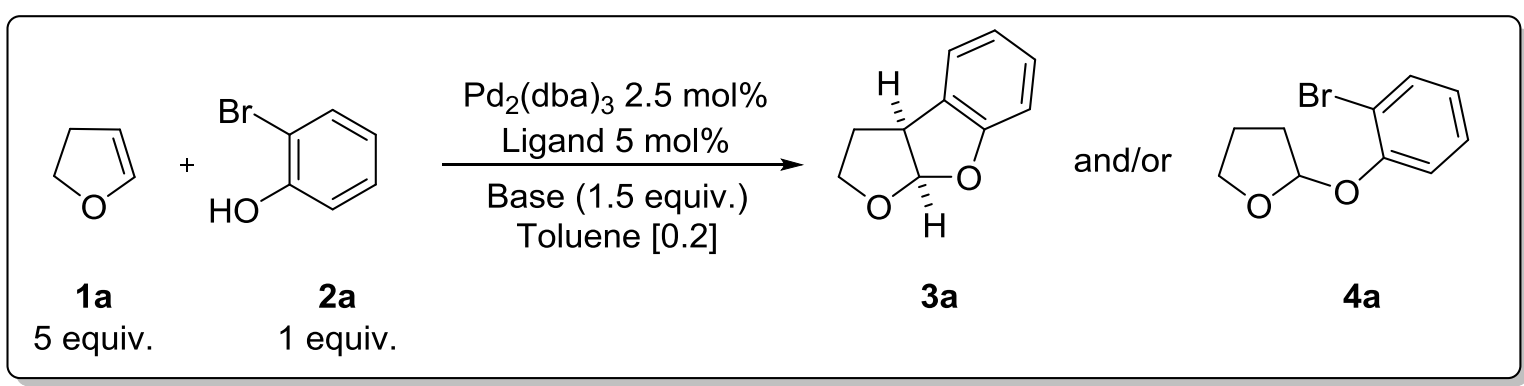

\begin{tabular}{|c|c|c|c|c|c|c|}
\hline & Ligand & $\mathrm{T}\left({ }^{\circ} \mathrm{C}\right)$ & Time & Base & $3 a(\% y)^{a, b}$ & $4 a(\% y)^{a, b}$ \\
\hline 1 & No ligand & 110 & $24 \mathrm{~h}$ & $\mathrm{NaOt}-\mathrm{Bu}$ & $\mathrm{nr}$ & $\mathrm{nr}$ \\
\hline 2 & No ligand & 110 & $24 \mathrm{~h}$ & $\mathrm{Li}_{2} \mathrm{CO}_{3}$ & nd & 92 \\
\hline 3 & No ligand ${ }^{c}$ & 110 & $24 \mathrm{~h}$ & $\mathrm{Li}_{2} \mathrm{CO}_{3}$ & nd & 97 \\
\hline 4 & No ligand ${ }^{c}$ & 110 & $24 \mathrm{~h}$ & $\mathrm{NaOt}-\mathrm{Bu}$ & $\mathrm{nr}$ & $\mathrm{nr}$ \\
\hline 5 & CPhos & 110 & $24 \mathrm{~h}$ & No Base & nd & 94 \\
\hline 6 & CPhos & 110 & $24 \mathrm{~h}$ & $\mathrm{~K}_{3} \mathrm{PO}_{4}$ & $\mathrm{nr}$ & $\mathrm{nr}$ \\
\hline 7 & CPhos & 110 & $24 \mathrm{~h}$ & LiHMDS & 20 & nd \\
\hline 8 & CPhos & 110 & $24 \mathrm{~h}$ & $\mathrm{Cs}_{2} \mathrm{CO}_{3}$ & $\mathrm{nr}$ & $\mathrm{nr}$ \\
\hline 9 & CPhos & 110 & $24 \mathrm{~h}$ & KOtBu & $\mathrm{nr}$ & $\mathrm{nr}$ \\
\hline 10 & CPhos & 110 & $24 \mathrm{~h}$ & $\mathrm{NaOt}-\mathrm{Bu}$ & 56 & nd \\
\hline 11 & CPhos & 110 & $24 \mathrm{~h}$ & $\mathrm{LiOt-Bu}$ & $\mathrm{nr}$ & $\mathrm{nr}$ \\
\hline 10 & $t$-BuXPhos & 110 & $24 \mathrm{~h}$ & $\mathrm{NaOt}-\mathrm{Bu}$ & 68 & nd \\
\hline 11 & SPhos & 110 & $24 \mathrm{~h}$ & $\mathrm{NaOt}-\mathrm{Bu}$ & 58 & nd \\
\hline 12 & TrixiePhos & 110 & $24 \mathrm{~h}$ & $\mathrm{NaOt}-\mathrm{Bu}$ & 36 & nd \\
\hline 13 & $t$-Bu-DavePhos & 110 & $24 \mathrm{~h}$ & $\mathrm{NaOt}-\mathrm{Bu}$ & 45 & nd \\
\hline 14 & RuPhos & 110 & $24 \mathrm{~h}$ & $\mathrm{NaOt}-\mathrm{Bu}$ & 71 & nd \\
\hline 15 & RuPhos & 110 & $4 \mathrm{~h}$ & $\mathrm{NaOt}-\mathrm{Bu}$ & 70 & nd \\
\hline 16 & RuPhos & 50 & $4 \mathrm{~h}$ & $\mathrm{NaOt}-\mathrm{Bu}$ & $<10 \%$ & nd \\
\hline 17 & RuPhos & 80 & $4 h^{d, e}$ & $\mathrm{NaOt}-\mathrm{Bu}$ & 73 & nd \\
\hline 18 & RuPhos-Pd-G3 ${ }^{f}$ & 80 & $4 \mathrm{~h}$ & $\mathrm{NaOt}-\mathrm{Bu}$ & 54 & nd \\
\hline
\end{tabular}




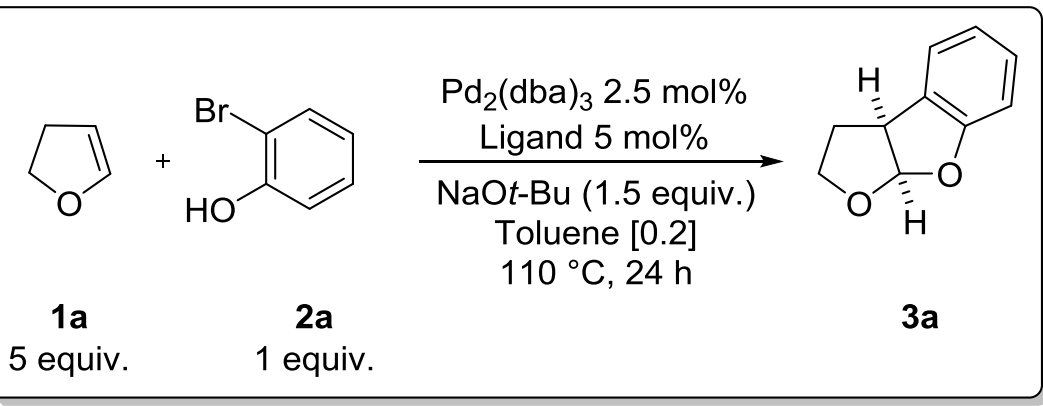<smiles>Cc1c(C)c(C)c(C(C)C)c(-c2c(C(C)C)cc(C(C)C)cc2P(C(C)C)C(C)C)c1C</smiles>

Me4 $t$-BuXPhos $\mathrm{nr}$

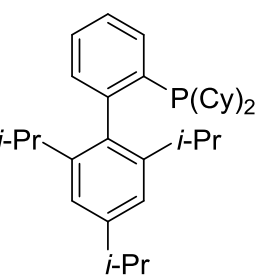

L3, XPhos $\mathrm{nr}$<smiles>CCCCC(C)(C)C</smiles>

L4, JohnPhos $\mathrm{nr}$<smiles>CCCCc1cc(C(C)C)cc(C(C)C)c1-c1ccccc1PC(C)C</smiles>

L6, $t$-BuXPhos $68 \%$ y<smiles>COc1ccc(OC)c(C(C)C)c1-c1c(C(C)C)cc(C(C)C)cc1P(C)C</smiles>

AdBrettPhos $\mathrm{nr}$<smiles>COc1cccc(OC)c1-c1ccccc1P(C)OC</smiles>

SPhos $58 \%$ y<smiles>CCCOc1ccccc1-c1c(OCCC)cccc1OCCC</smiles>

L8, RuPhos $73 \%$ y<smiles>COc1ccc(OC)c(-c2c(C(C)C)cc(C(C)C)cc2P(C)C)c1[PH3+]</smiles>

$\mathrm{Ar}=3,5-\left(\mathrm{CF}_{3}\right)_{2}-\mathrm{C}_{6} \mathrm{H}_{3}$

$t$-BuBrettPhos $\mathrm{nr}$
JackiePhos

$\mathrm{nr}$<smiles>CC(C)(C)c1ccc2ccccc2c1-c1cccc2ccccc12</smiles>

L7, TrixiePhos $36 \%$ y<smiles>CCP(C)c1ccccc1-c1ccccc1C</smiles>

MePhos

$\mathrm{nr}$

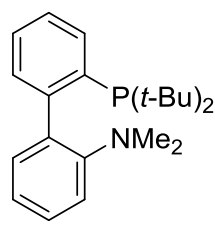

L5, $t$-BuDavePhos $45 \%$ y<smiles>CCCc1ccccc1-c1ccccc1</smiles>

CyJohnPhos $\mathrm{nr}$

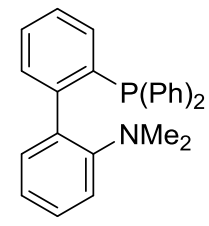

PhDavePhos

$\mathrm{nr}$<smiles>CNc1ccccc1-c1ccccc1P(C)C</smiles>

L2, DavePhos $\mathrm{nr}$<smiles>CCCc1ccccc1-c1c(N(C)C)cccc1N(C)C</smiles>

L1, CPhos $56 \%$ y 
2.2 Optimization of the carboetherification of 2,3-dhf with 2-bromophenol and a $\mathrm{Pd}^{0}$ source (enantioselective version)

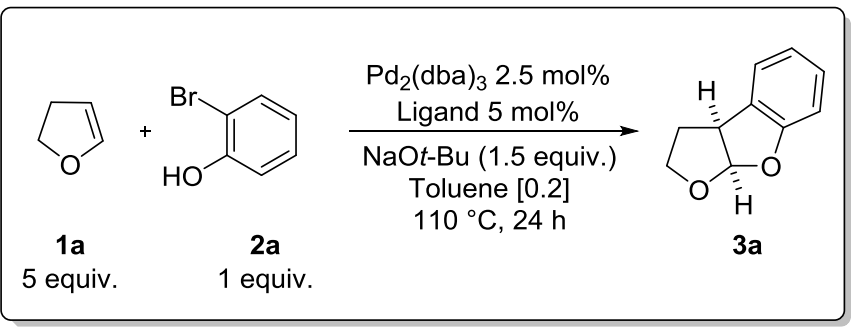<smiles>CC1(C)OC2C(c3ccccc3)OP(OC3CCCCC3c3ccccc3)OC(c3ccccc3)(c3ccccc3)C2O1</smiles>

L11

$\mathrm{nr}$

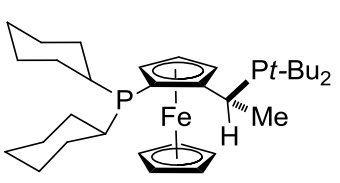

$\mathrm{nr}$

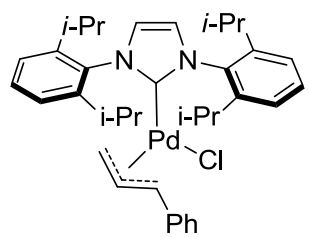

$\mathrm{nr}$<smiles>N#[P+]C1=C(c2c(C(N)=O)ccc3c2OCO3)C2OCOC2C=C1</smiles>

$\mathrm{Ar}=3,5-(t-\mathrm{Bu})_{2}-4-\mathrm{MeO}-\mathrm{C}_{6} \mathrm{H}_{2}$

$\mathrm{nr}$

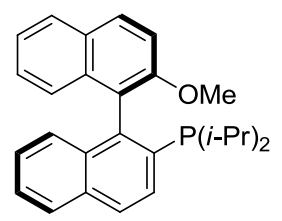

$58 \%$ y

$13 \%$ ee<smiles></smiles>

$\mathrm{nr}$<smiles>CC(C)(C)P1Cc2ccc3ccccc3c2-c2c(ccc3ccccc23)C1Cc1ccccn1</smiles>

$\mathrm{nr}$

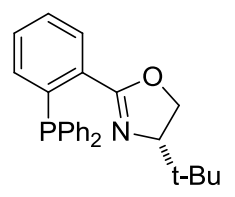

nr

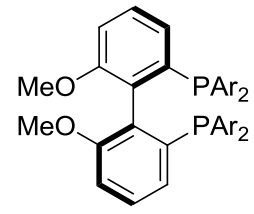

$\mathrm{Ar}=3,5-(t-\mathrm{Bu})_{2}-4-\mathrm{MeO}-\mathrm{C}_{6} \mathrm{H}_{2}$

$$
\text { (R)-L18 }
$$

$\mathrm{nr}$

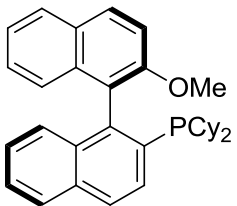

L9

$64 \%$ y

$5 \%$ ee<smiles></smiles>

$\mathrm{nr}$

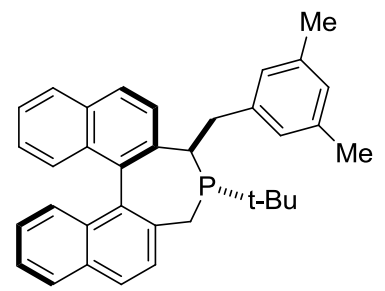

$\mathrm{nr}$

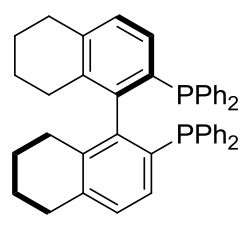

$\mathrm{nr}$

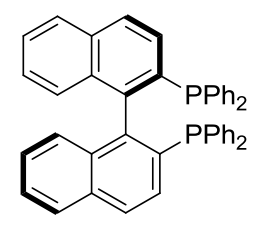

L12

$\mathrm{nr}$

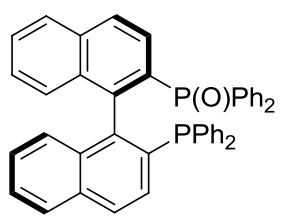

L13

$15 \%$ y

$27 \%$ ee<smiles>CC(c1ccccc1)N(C(c1ccccc1)c1ccccc1)p1oc2ccc3ccccc3c2c2c(ccc3ccccc32)o1</smiles>

$\mathrm{nr}$<smiles>COc1cccc(OC)c1-c1ccccc1P1Cc2ccc3ccccc3c2-c2c(ccc3ccccc23)C1</smiles>

$\mathrm{nr}$<smiles>FC(F)(F)Oc1ccc2c(c1-c1c(-c3ccccc3)ccc3c1OC(F)(F)O3)OC(F)(F)O2</smiles>

$\mathrm{nr}$<smiles>CCCCPc1ccc2ccccc2c1-c1c(OCCC)ccc2ccccc12</smiles>

$\mathrm{nr}$

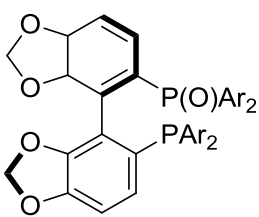

$\mathrm{Ar}=3,5-(t-\mathrm{Bu})_{2}-4-\mathrm{MeO}-\mathrm{C}_{6} \mathrm{H}_{2}$

L14

$8 \%$ y $92 \%$ ee 
2.3 Optimization of the carboetherification of 2,3-dhf with 2-bromophenol and a Pd" source (enantioselective version)

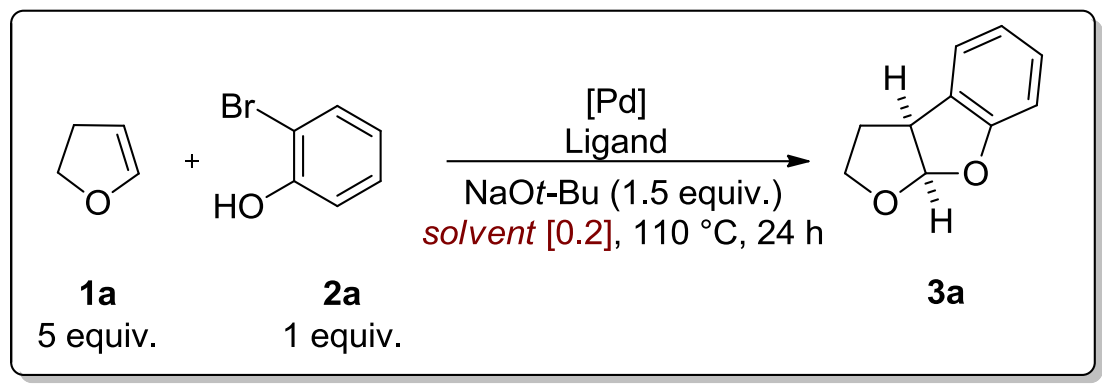

\begin{tabular}{|c|c|c|c|c|c|c|}
\hline & $\begin{array}{l}{[\mathrm{Pd}]} \\
(5 \mathrm{~mol} \%)\end{array}$ & $\begin{array}{l}\text { Ligand } \\
\text { (5 mol\%) }\end{array}$ & $\begin{array}{l}\text { Solvent } \\
{[\mathrm{M}]}\end{array}$ & $\begin{array}{l}\mathrm{H}_{2} \mathrm{O} \\
(\mathrm{mol} \%)\end{array}$ & $\begin{array}{l}3 a \\
(\% y)^{a}\end{array}$ & $\begin{array}{l}3 a \\
(\% e e)^{b}\end{array}$ \\
\hline 1 & $\mathrm{Pd}_{2}(\mathrm{dba})_{3}$ & $(R)$-BINAP & Toluene & - & $\mathrm{nr}$ & - \\
\hline 2 & $\mathrm{Pd}(\mathrm{OAc})_{2}$ & $(R)-\mathrm{BINAP}^{\mathrm{c}}$ & Toluene & - & $\mathrm{nr}$ & - \\
\hline 3 & $\mathrm{Pd}(\mathrm{OAC})_{2}$ & $(R)$-BINAP & Toluene & - & $<5$ & $<5$ \\
\hline 4 & $\mathrm{Pd}(\mathrm{OAc})_{2}$ & $(R)$-DTBM-SEGPHOS & Toluene & - & 16 & 90 \\
\hline 5 & $\mathrm{Pd}(\mathrm{OAc})_{2}$ & $(R)$-DTBM-SEGPHOS & $\begin{array}{l}\text { Toluene/t-Amyl alcohol } \\
(2: 1)\end{array}$ & - & 21 & 90 \\
\hline 6 & $\mathrm{Pd}(\mathrm{OAc})_{2}$ & $(R)$-DTBM-SEGPHOS & t-Amyl alcohol & - & 11 & 81 \\
\hline 7 & $\mathrm{Pd}(\mathrm{OAc})_{2}$ & $(R)$-DTBM-SEGPHOS & Toluene & 20 & 31 & 83 \\
\hline 8 & $\mathrm{Pd}(\mathrm{OAc})_{2}$ & $(R)$-DTBM-SEGPHOS & Toluene & 40 & 40 & 85 \\
\hline 9 & $\mathrm{Pd}(\mathrm{OAc})_{2}$ & $(R)$-DTBM-SEGPHOS & Toluene & 50 & 31 & 80 \\
\hline 10 & $\mathrm{Pd}(\mathrm{OAc})_{2}$ & $(R)$-DTBM-SEGPHOS & Toluene & 60 & 35 & 81 \\
\hline 11 & $\mathrm{Pd}(\mathrm{OAc})_{2}$ & (S)-DTBM-MeO-BIPHEP & Toluene & 40 & 74 & 92 \\
\hline 12 & $\mathrm{Pd}(\mathrm{OAc})_{2}$ & (S)-DTBM-MeO-BIPHEP & Toluene & - & $40^{d}$ & 95 \\
\hline
\end{tabular}

a Isolated yield after column chromatography; ${ }^{b}$ Enantiomeric excess determined by HPLC or GC; ${ }^{c}$ Using a 10 mol\% ${ }^{\mathrm{d}}$ No full consumption of starting 2-Bromophenol. 


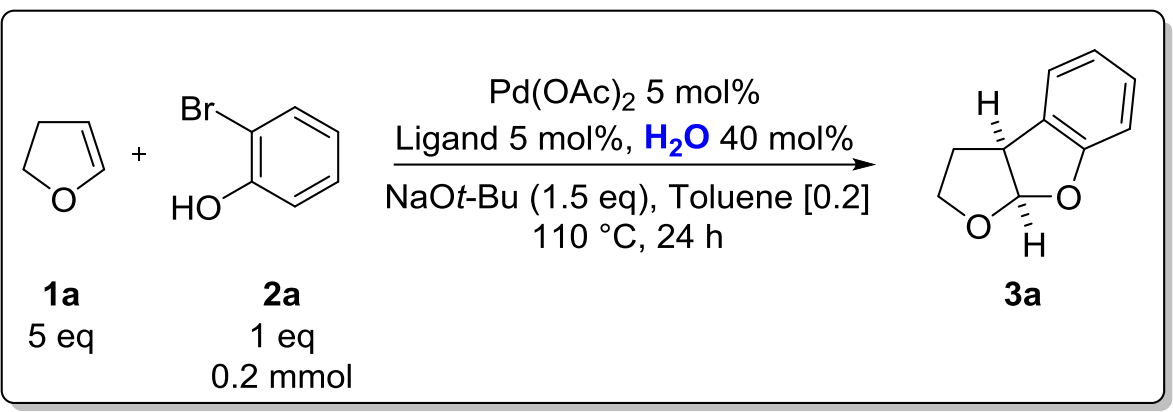

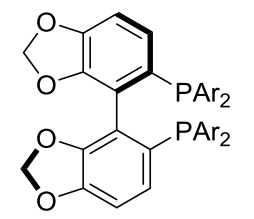

$\mathrm{Ar}=3,5-(\mathrm{Me})_{2}-\mathrm{C}_{6} \mathrm{H}_{3}$

$\mathrm{nr}$

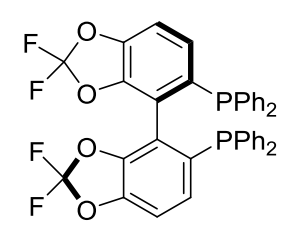

$\mathrm{nr}$

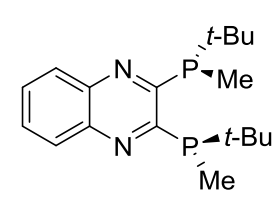

$\mathrm{nr}$<smiles>C[C@@H]1CC[C@@H](C)P1c1ccccc1P1[C@H](C)CC[C@H]1C</smiles>

$\mathrm{nr}$

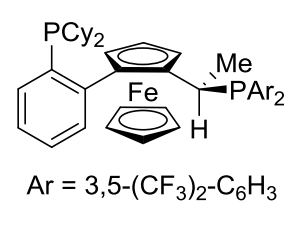

$\mathrm{nr}$

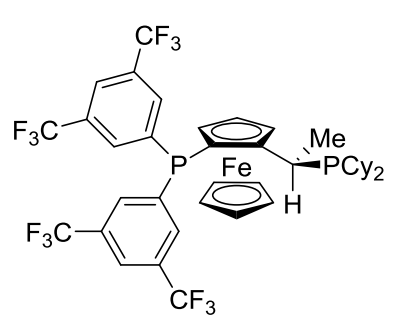

$\mathrm{nr}$

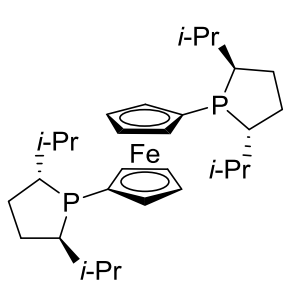

$\mathrm{nr}$

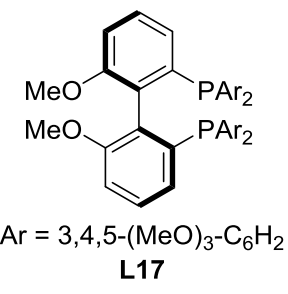

$31 \%$ y $65 \%$ ee

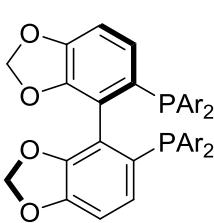

$\mathrm{Ar}=3,5-(t-\mathrm{Bu})_{2}-4-\mathrm{MeO}-\mathrm{C}_{6} \mathrm{H}_{2}$

$40 \%$ y $85 \%$ ee<smiles>COc1cccc(P(C)C)c1-c1c(OC)cccc1P(C)C</smiles>

$\mathrm{Ar}=3,5-(t-\mathrm{Bu})_{2}-\mathrm{C}_{6} \mathrm{H}_{3}$

L15

$76 \%$ y

$90 \%$ ee

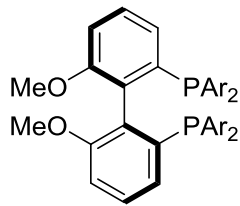

$\mathrm{Ar}=3,5-(t-\mathrm{Bu})_{2}-4-\mathrm{NMe}_{2}-\mathrm{C}_{6} \mathrm{H}_{2}$

L16

$66 \%$ y

$90 \%$ ee

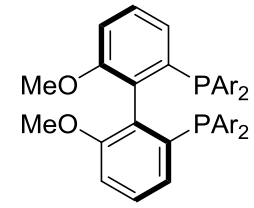

$\mathrm{Ar}=3,5-(t-\mathrm{Bu})_{2}-4-\mathrm{MeO}-\mathrm{C}_{6} \mathrm{H}_{2}$

L18

$74 \%$ y

$92 \%$ ee 


\section{Experimental procedures}

3.1 General procedure for the racemic intermolecular carboetherification of 2,3-dihydrofurans using 2-bromophenol derivatives (GP1)

In a glovebox, a $5 \mathrm{~mL}$ Young valve Schlenk was charged with $\mathrm{Pd}_{2}(\mathrm{dba})_{3}(11.4 \mathrm{mg}, 0.0125 \mathrm{mmol}, 2.5$ mol\%), RuPhos (11.6 mg, $0.025 \mathrm{mmol}, 5 \mathrm{~mol} \%$ ) and $2.5 \mathrm{~mL}$ of degassed toluene. The Schlenk was taken outside the glovebox, connected to a two-manifold line and the mixture was stirred at r.t. for $10 \mathrm{~min}$. Next, a 2-bromophenol derivative (0.5 mmol, 1 equiv.), NaOt-Bu (72 mg, $0.75 \mathrm{mmol}, 1.5$ equiv.) and 2,3-dihydrofuran ( $0.19 \mathrm{~mL}, 2.5 \mathrm{mmol}, 5$ equiv.) were added successively under a flow of $\mathrm{N}_{2}$ gas. The sealed reaction tube was immerged in a $80^{\circ} \mathrm{C}$ pre-heated oil bath for $6 \mathrm{~h}$. After cooling to r.t., the reaction mixture was poured into $\mathrm{Et}_{2} \mathrm{O}(5 \mathrm{~mL})$ under vigorous stirring, and the resulting precipitate was removed passing the solution through a short pad of Celite ${ }^{\circledR}$. The filtrate was concentrated to dryness and the resulting oil was subjected to flash chromatography (Pentane:Et $\mathrm{E}_{2} \mathrm{O}$ ).

\subsection{General procedure for the enantioselective intermolecular carboetherification of 2,3- dihydrofurans using 2-bromophenol derivatives (GP2)}

In a glovebox, a $5 \mathrm{~mL}$ Young valve Schlenk was charged with $\mathrm{Pd}(\mathrm{OAc})_{2}(2.2 \mathrm{mg}, 0.01 \mathrm{mmol}, 5 \mathrm{~mol} \%)$, (S)-DTBM-MeO-BIPHEP (11.5 mg, $0.01 \mathrm{mmol}, 5 \mathrm{~mol} \%$ ) and $1.0 \mathrm{~mL}$ of degassed toluene. The Schlenk was taken outside the glovebox, connected to a two-manifold line and degassed $\mathrm{H}_{2} \mathrm{O}(1.4 \mu \mathrm{L}, 0.08$ mmol, 40 mol\%) was added under a flow of $\mathrm{N}_{2}$ gas. After stirring at r.t for $10 \mathrm{~min}$, the mixture was stirred first at $110{ }^{\circ} \mathrm{C}$ for $2.5 \mathrm{~min}$ and then at r.t. for another $2.5 \mathrm{~min}$ before the sequential addition of the bromophenol derivative ( $0.2 \mathrm{mmol}, 1$ equiv.), $\mathrm{NaOt}$ - $\mathrm{Bu}(29 \mathrm{mg}, 0.3 \mathrm{mmol}, 1.5$ equiv.) and 2,3dihydrofuran ( $76 \mu \mathrm{L}, 1.0 \mathrm{mmol}, 5$ equiv.) under a flow of $\mathrm{N}_{2}$ gas. The sealed reaction tube was immerged in a $110{ }^{\circ} \mathrm{C}$ pre-heated oil bath for $24 \mathrm{~h}$. After cooling to r.t., the reaction mixture was poured into $\mathrm{Et}_{2} \mathrm{O}(5 \mathrm{~mL})$ under vigorous stirring, and the resulting precipitate was removed passing the solution trough a short pad of Celite ${ }^{\circledR}$. The filtrate was concentrated to dryness and the resulting oil was subjected to flash chromatography (Pentane:Et ${ }_{2} \mathrm{O}$ ).

Note: The reaction outcome (yield and enantioselectivity) is particularly sensitive to the amount of water in the reaction media. Therefore, the use of perfectly dried toluene, 2,3-dihydrofurans and 2bromophenol derivatives is necessary to avoid discrepancies with the reported experimental values. 


\subsection{Synthesis and characterization of rac-2-(p-tolyl)-2,3-dihydrofuran (1c)}<smiles>Cc1ccc(Br)cc1</smiles>

(5 equiv.)

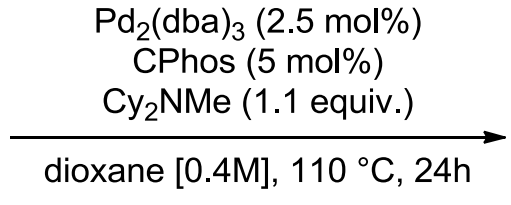

dioxane $[0.4 \mathrm{M}], 110^{\circ} \mathrm{C}, 24 \mathrm{~h}$

$1 c$

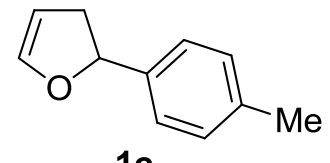

1c

In a glovebox, a $50 \mathrm{~mL}$ Young valve Schlenk was charged with $\mathrm{Pd}_{2}(\mathrm{dba})_{3}(126 \mathrm{mg}, 0.1375 \mathrm{mmol}, 2.5$ mol\%), CPhos (120 mg, $0.275 \mathrm{mmol}, 5 \mathrm{~mol} \%$ ) and $10.0 \mathrm{~mL}$ of distilled and degassed 1,4-dioxane. The Schlenk was taken outside the glovebox, connected to a two-manifold line and the mixture was stirred at r.t. for $10 \mathrm{~min}$. Next, 4-bromotoluene (0.68 mL, $5.5 \mathrm{mmol}, 1$ equiv.), $\mathrm{Cy}_{2} \mathrm{NMe} \mathrm{(1.3} \mathrm{mL,} 6.0$ mmol, 1.1 equiv.) and 2,3-dihydrofuran (1a) $(2.0 \mathrm{~mL}, 27.5 \mathrm{mmol}, 5$ equiv.,) were added consecutively under a flow of $\mathrm{N}_{2}$ gas. The sealed reaction tube was immerged in a $110^{\circ} \mathrm{C}$ pre-heated oil bath for 24 h. After cooling to r.t., the reaction mixture was poured into $\mathrm{Et}_{2} \mathrm{O}(20 \mathrm{~mL})$ under vigorous stirring, and the resulting precipitate was removed passing the solution through a short pad of Celite ${ }^{\circledR}$. The filtrate was concentrated to dryness to give an oil which was directly subjected to flash chromatography (Pentane:Et ${ }_{2} \mathrm{O}=80: 1$ ) affording rac-2-(p-tolyl)-2,3-dihydrofuran (1c) as a pale yellow oil ( $55 \%$ yield, $0.48 \mathrm{~g}$ ) with $R_{f}=0.33$.<smiles></smiles>

${ }^{1} \mathrm{H}-\mathrm{NMR}\left(\mathrm{CDCl}_{3}, 400 \mathrm{MHz}\right): \delta(\mathrm{ppm})=2.10(\mathrm{~s}, 3 \mathrm{H}, \mathrm{H}-10), 2.43(\mathrm{~m}, 1 \mathrm{H}, \mathrm{H}-4), 2.72\left(\mathrm{~m}, 1 \mathrm{H}, \mathrm{H}-4^{\prime}\right), 4.68$ $(\mathrm{m}, 1 \mathrm{H}, \mathrm{H}-3), 5.37\left(\mathrm{dd},{ }^{3} \mathrm{~J}_{\mathrm{H}-\mathrm{H}}=10.7 \mathrm{~Hz},{ }^{3} \mathrm{~J}_{\mathrm{H}-\mathrm{H}}=8.5 \mathrm{~Hz}, 1 \mathrm{H}, \mathrm{H}-5\right), 6.31(\mathrm{~m}, 1 \mathrm{H}, \mathrm{H}-2), 6.99(\mathrm{~m}, 2 \mathrm{H}, \mathrm{H}-8)$, $7.21(\mathrm{~m}, 2 \mathrm{H}, \mathrm{H}-7) ;{ }^{13} \mathrm{C}\left\{{ }^{1} \mathrm{H}\right\}-\mathrm{NMR}\left(\mathrm{CDCl}_{3}, 100 \mathrm{MHz}\right): \delta(\mathrm{ppm})=21.1$ (C-10), 38.2 (C-4), 82.6 (C-5), 98.8 (C-3), 126.0 (C-7), 129.4 (C-8), 137.1 (C-9), 140.9 (C-6), 145.9 (C-2); IR spectrum (neat) $\left(\mathrm{cm}^{-1}\right.$ ) = 2923, 1619, 1514, 1452, 1135, 1049, 932, 826, 807, 701, 552.

Spectral data are in agreement with previous characterization. ${ }^{2}$ 


\subsection{Synthesis and characterization of trans-rac-3-methyl-2-(p-tolyl)-2,3-dihydrofuran (1d)}

4-Methyl-2,3-dihydrofuran was synthesized following previously reported procedures for the reduction of lactones ${ }^{3}$ and the dehydration of cyclic hemiacetals. ${ }^{4}$

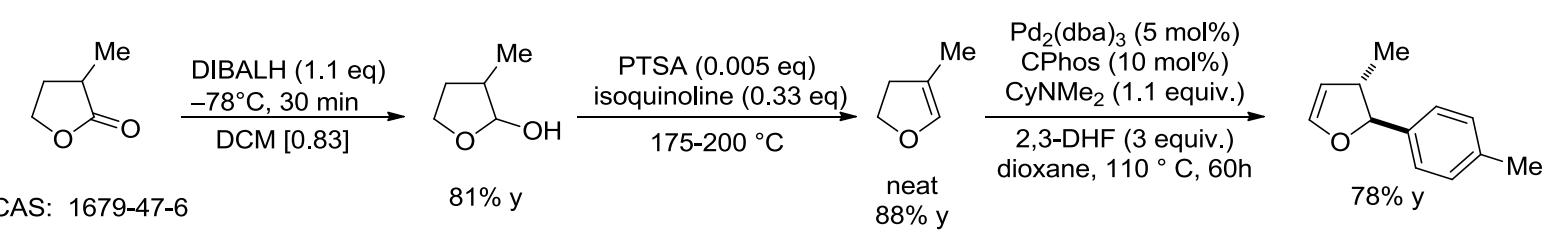

Arylation step: In a glovebox, a $10 \mathrm{~mL}$ Young valve Schlenk was charged with $\operatorname{Pd}_{2}(\mathrm{dba})_{3}(142 \mathrm{mg}$, $0.155 \mathrm{mmol}, 5 \mathrm{~mol} \%$ ), CPhos (135 mg, $0.310 \mathrm{mmol}, 10 \mathrm{~mol} \%$ ) and $3.0 \mathrm{~mL}$ of distilled and degassed 1,4-dioxane. The Schlenk was taken outside the glovebox, connected to a two-manifold line and the mixture was stirred at r.t. for $10 \mathrm{~min}$. Next, 4-bromotoluene $\left(0.38 \mathrm{~mL}, 3.1 \mathrm{mmol}, 1\right.$ equiv.), $\mathrm{Cy}_{2} \mathrm{NMe}$ ( $0.73 \mathrm{~mL}, 3.4 \mathrm{mmol}, 1.1$ equiv.) and 4-methyl-2,3-dihydrofuran ( $0.8 \mathrm{~g}, 9.3 \mathrm{mmol}, 3$ equiv.) were added consecutively under a flow of $\mathrm{N}_{2}$ gas. The sealed reaction tube was immerged in a $110{ }^{\circ} \mathrm{C}$ preheated oil bath for $60 \mathrm{~h}$. After cooling to r.t., the reaction mixture was poured into $\mathrm{Et}_{2} \mathrm{O}(10 \mathrm{~mL})$ under vigorous stirring, and the resulting precipitate was removed passing the solution through a short pad of Celite ${ }^{\circledast}$. The filtrate was concentrated to dryness to give an oil which was directly subjected to flash chromatography (Pentane:Et ${ }_{2} \mathrm{O}$ 50:1) affording trans-rac-3-methyl-2-(p-tolyl)-2,3dihydrofuran (1d) as a pale yellow oil (78\% yield, $0.41 \mathrm{~g})$ with $R_{f}=0.40$.<smiles>COC1C=COC1c1ccc([N+](=O)[O-])cc1</smiles>

${ }^{1} \mathrm{H}-\mathrm{NMR}\left(\mathrm{CDCl}_{3}, 400 \mathrm{MHz}\right): \delta(\mathrm{ppm})=1.00\left(\mathrm{~d},{ }^{3} \mathrm{~J}_{\mathrm{H}-\mathrm{H}}=6.7 \mathrm{~Hz}, 3 \mathrm{H}, \mathrm{H}-11\right), 2.11(\mathrm{~s}, 3 \mathrm{H}, \mathrm{H}-10), 3.04(\mathrm{~m}, 1 \mathrm{H}$, $\mathrm{H}-4), 4.71(\mathrm{~m}, 1 \mathrm{H}, \mathrm{H}-3), 4.90\left(\mathrm{~d},{ }^{3} \mathrm{~J}_{\mathrm{H}-\mathrm{H}}=7.8 \mathrm{~Hz}, 3 \mathrm{H}, \mathrm{H}-5\right), 6.32(\mathrm{~m}, 1 \mathrm{H}, \mathrm{H}-2), 7.00(\mathrm{~m}, 2 \mathrm{H}, \mathrm{H}-8), 7.25(\mathrm{~m}$, $2 \mathrm{H}, \mathrm{H}-7) ;{ }^{13} \mathrm{C}\left\{{ }^{1} \mathrm{H}\right\}-\mathrm{NMR}\left(\mathrm{CDCl}_{3}, 100 \mathrm{MHz}\right): \delta(\mathrm{ppm})=20.7$ (C-11), 21.1 (C-10), 46.4 (C-4), 90.5 (C-5), 105.5 (C-3), 125.9 (C-7), 129.4 (C-8), 137.3 (C-9), 140.2 (C-6), 145.4 (C-2); IR spectrum (neat) (cm ${ }^{-1}$ ) = $2923,1618,1139,1098,964,933,803,715$.

Spectral data are in agreement with previous characterization. ${ }^{5}$ 


\section{Characterization data}<smiles>COC1OCCC2OC1Oc1ccccc12</smiles>

\section{$(3 a S, 8 a R)-2,3,3 a, 8 a-T e t r a h y d r o f u r o[2,3-b]$ benzofuran (3a)}

Prepared according to GP2 using 2,3-dihydrofuran (1a) (76 $\mu \mathrm{L}, 1 \mathrm{mmol}$ ) and 2-bromophenol (2a) (23 $\mu \mathrm{L}, 0.2 \mathrm{mmol}$ ). Isolated by column chromatography (Pentane: $\mathrm{Et}_{2} \mathrm{O} 40: 1$ ) as a pale yellow oil (74\% yield, $92 \%$ ee) with $\mathrm{R}_{\mathrm{f}}=0.28 ;{ }^{1} \mathrm{H}-\mathrm{NMR}\left(\mathbf{C}_{6} \mathrm{D}_{6}, 400 \mathrm{MHz}\right): \delta(\mathrm{ppm})=1.44(\mathrm{~m}, 1 \mathrm{H}, \mathrm{H}-3), 1.60(\mathrm{~m}, 1 \mathrm{H}, \mathrm{H}-$ $\left.3^{\prime}\right), 3.26(\mathrm{~m}, 1 \mathrm{H}, \mathrm{H}-4), 3.34\left(\mathrm{ddd},{ }^{2} \mathrm{~J}_{\mathrm{H}-\mathrm{H}}=11.9 \mathrm{~Hz},{ }^{3} \mathrm{~J}_{\mathrm{H}-\mathrm{H}}=8.6 \mathrm{~Hz},{ }^{3} \mathrm{~J}_{\mathrm{H}-\mathrm{H}}=4.9 \mathrm{~Hz}, 1 \mathrm{H}, \mathrm{H}-2\right), 3.61\left(\mathrm{t},{ }^{3} \mathrm{~J}_{\mathrm{H}-\mathrm{H}}=\right.$ $\left.8.1 \mathrm{~Hz}, 1 \mathrm{H}, \mathrm{H}-2^{\prime}\right), 6.08\left(\mathrm{~d},{ }^{3} J_{\mathrm{H}-\mathrm{H}}=5.7 \mathrm{~Hz}, 1 \mathrm{H}, \mathrm{H}-12\right), 6.75(\mathrm{~m}, 1 \mathrm{H}, \mathrm{H}-6), 6.82(\mathrm{~m}, 2 \mathrm{H}, \mathrm{H}-7$ and H-8), 7.06 (m, $1 \mathrm{H}, \mathrm{H}-9) ;{ }^{13} \mathrm{C}\left\{{ }^{1} \mathrm{H}\right\}-\mathrm{NMR}\left(\mathrm{C}_{6} \mathrm{D}_{6}, 100 \mathrm{MHz}\right): \delta(\mathrm{ppm})=33.6$ (C-3), 46.6 (C-4), 66.8 (C-2), 109.4 (C-7 or C-8), 111.3 (C-12), 121.1 (C-6), 124.8 (C-7 or C-8), 128.0 (C-5), 129.0 (C-9), 160.39 (C-10); GC-MS (EI): $\left(\mathrm{C}_{10} \mathrm{H}_{10} \mathrm{O}_{2}\right), 162.1\left(100, \mathrm{M}^{+}\right), 147.1\left(47, \mathrm{M}^{+}-15\right), 105.1\left(46, \mathrm{M}^{+}-57\right), 77.1\left(43, \mathrm{M}^{+}-85\right)$; IR spectrum (neat) $\left(\mathrm{cm}^{-1}\right)=2978,1597,1477,1461,1323,1242,1187,1074,1016,952,923,819,747,650,550$; $[\alpha]^{23}{ }_{D}=-142.2\left(c \quad 0.85, \mathrm{CH}_{2} \mathrm{Cl}_{2}\right.$ ); HPLC: IC, $205 \mathrm{~nm}$, Hexane:iPrOH, 98:2, $1 \mathrm{~mL} / \mathrm{min}, 30{ }^{\circ} \mathrm{C}, t_{\mathrm{R} 1}=10.1$ and $t_{\mathrm{R} 2}=12.6 \mathrm{~min}$.

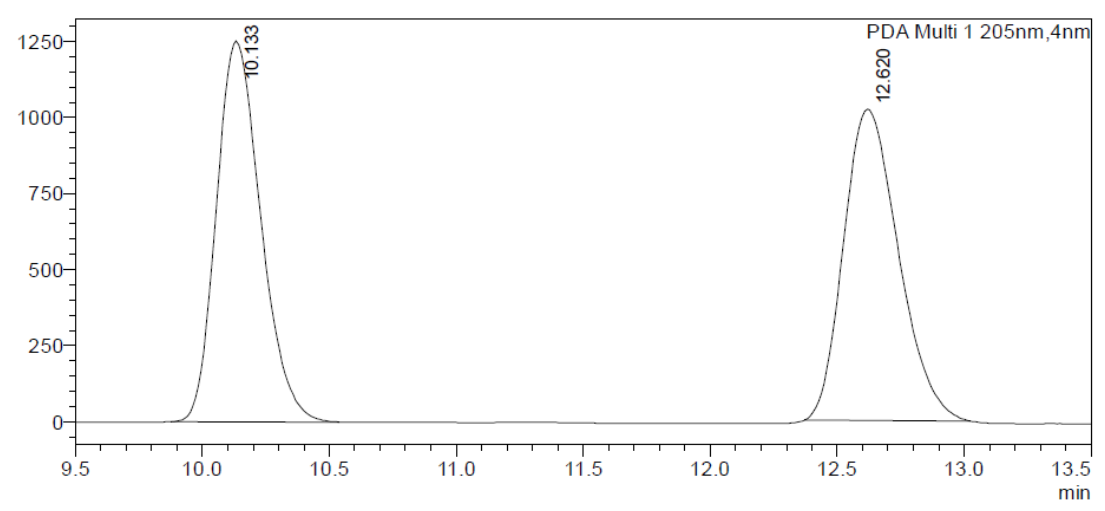

<Peak Table>
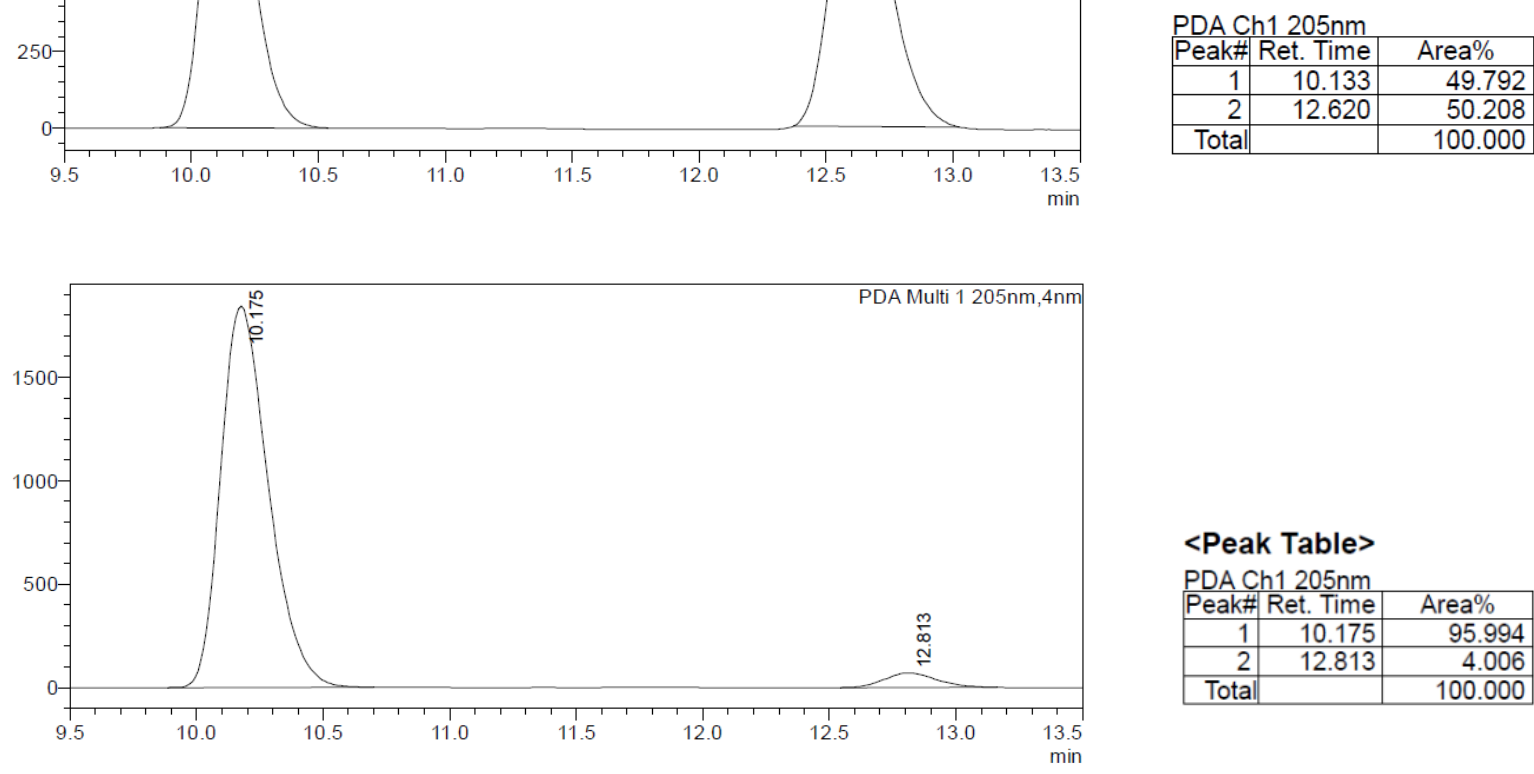
<smiles>COc1ccc2c(c1)C1CCOC1OCCO2</smiles>

\section{(3aS,8aR)-5-Methoxy-2,3,3a,8a-tetrahydrofuro[2,3-b]benzofuran (3b)}

Prepared according to GP2 using 2,3-dihydrofuran (1a) (76 $\mu \mathrm{L}, 1 \mathrm{mmol}$ ) and 2-bromo-4methoxyphenol (2b) $(40.6 \mathrm{mg}, 0.2 \mathrm{mmol})$. Isolated by column chromatography (Pentane:Et ${ }_{2} \mathrm{O}$ 10:1) as a pale yellow oil (73\% yield, $94 \%$ ee) with $\mathrm{R}_{\mathrm{f}}=0.20 ;{ }^{1} \mathbf{H}-\mathbf{N M R}\left(\mathbf{C}_{6} \mathbf{D}_{6}, 400 \mathrm{MHz}\right): \delta(\mathrm{ppm})=1.42(\mathrm{~m}$, $1 \mathrm{H}, \mathrm{H}-3), 1.62\left(\mathrm{~m}, 1 \mathrm{H}, \mathrm{H}-3^{\prime}\right), 3.25(\mathrm{~m}, 1 \mathrm{H}, \mathrm{H}-4), 3.34(\mathrm{~s}, 3 \mathrm{H}, \mathrm{H}-13), 3.44$ (ddd, ${ }^{2} J_{\mathrm{H}-\mathrm{H}}=12.0 \mathrm{~Hz},{ }^{3} \mathrm{~J}_{\mathrm{H}-\mathrm{H}}=8.6$ $\left.\mathrm{Hz},{ }^{3} J_{\mathrm{H}-\mathrm{H}}=5.0 \mathrm{~Hz}, 1 \mathrm{H}, \mathrm{H}-2\right), 3.64\left(\mathrm{t},{ }^{3} J_{\mathrm{H}-\mathrm{H}}=8.0 \mathrm{~Hz}, 1 \mathrm{H}, \mathrm{H}-2^{\prime}\right), 6.12\left(\mathrm{~d},{ }^{3} J_{\mathrm{H}-\mathrm{H}}=5.6 \mathrm{~Hz}, 1 \mathrm{H}, \mathrm{H}-12\right), 6.51(\mathrm{~d}$, $\left.{ }^{3} J_{\mathrm{H}-\mathrm{H}}=8.6 \mathrm{~Hz},{ }^{4} J_{\mathrm{H}-\mathrm{H}}=2.8 \mathrm{~Hz}, 1 \mathrm{H}, \mathrm{H}-8\right), 6.58\left(\mathrm{~d},{ }^{4} J_{\mathrm{H}-\mathrm{H}}=2.4 \mathrm{~Hz}, 1 \mathrm{H}, \mathrm{H}-6\right), 6.73\left(\mathrm{~d},{ }^{3} J_{\mathrm{H}-\mathrm{H}}=8.6 \mathrm{~Hz}, 1 \mathrm{H}, \mathrm{H}-9\right.$ ); ${ }^{13} \mathrm{C}\left\{{ }^{1} \mathrm{H}\right\}-N M R\left(\mathrm{C}_{6} \mathrm{D}_{6}, 100 \mathrm{MHz}\right): \delta(\mathrm{ppm})=33.6$ (C-3), 47.2 (C-4), 55.5 (C-13), 66.9 (C-2), 109.3 (C-9), 111.4 (C-6), 111.6 (C-12), 113.6 (C-8), 129.2 (C-4), 154.4 (C-7 or C-10), 155.0 (C-7 or C-10); GC-MS (EI): $\left(\mathrm{C}_{11} \mathrm{H}_{12} \mathrm{O}_{3}\right), 192.1\left(100, \mathrm{M}^{+}\right), 177.1\left(20, \mathrm{M}^{+}-15\right), 163.1$ (17, $\left.\mathrm{M}^{+}-29\right), 149.1\left(22, \mathrm{M}^{+}-43\right), 91.1$ $\left(22, \mathrm{M}^{+}-101\right)$; IR spectrum (neat) $\left(\mathrm{cm}^{-1}\right)=2979,1621,1512,1433,1341,1278,1197,1181,1146$, 1114, 1074, 1029, 959, 917, 825; $[\alpha]^{23}=-166.8$ (c 0.54, $\mathrm{CH}_{2} \mathrm{Cl}_{2}$ ); HPLC: OJ-H, $205 \mathrm{~nm}$, Hexane:iPrOH, $98: 2,1 \mathrm{~mL} / \mathrm{min}, 30^{\circ} \mathrm{C}, t_{\mathrm{R} 1}=19.2$ and $t_{\mathrm{R} 2}=24.4 \mathrm{~min}$.
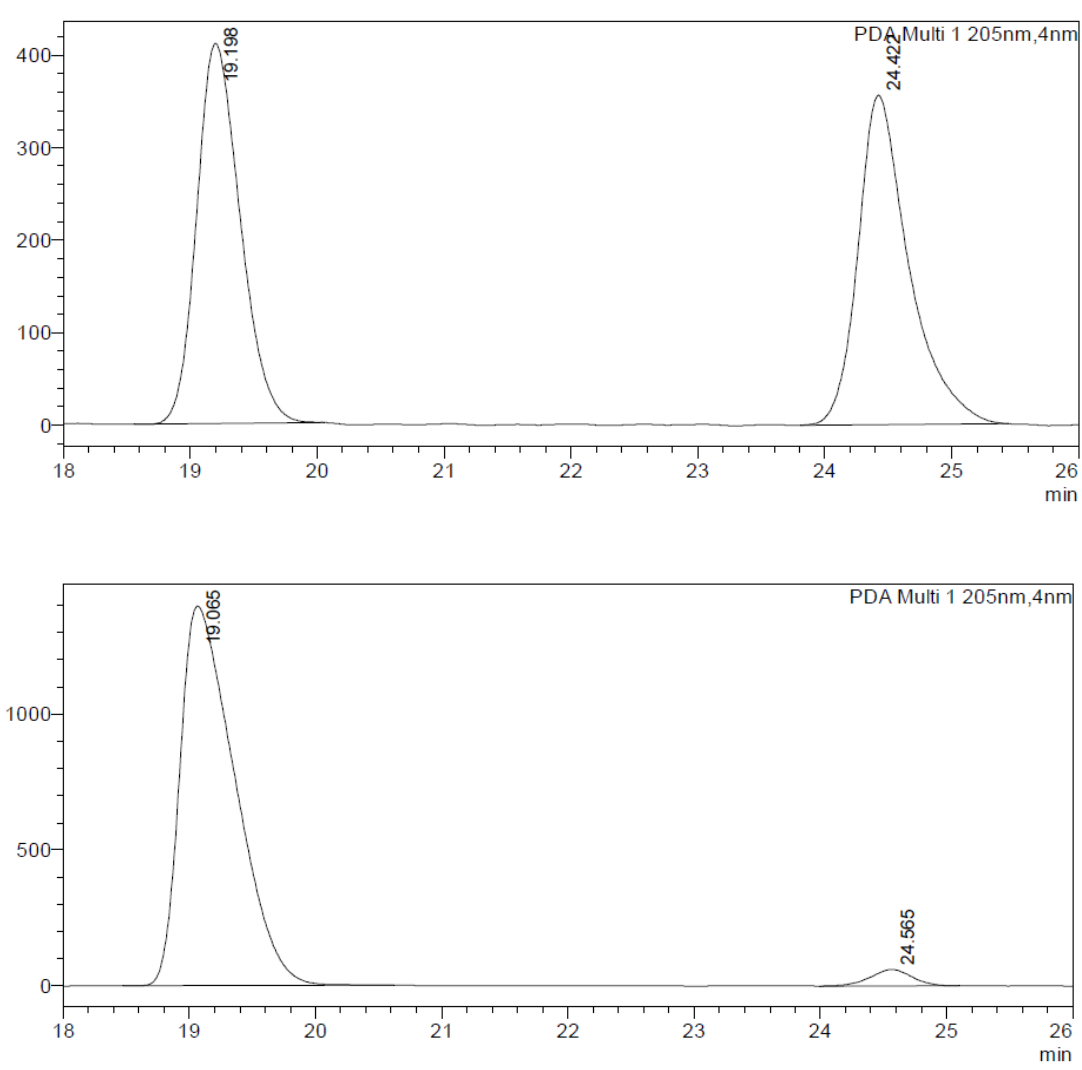

<Peak Table> PDA Ch1 205nm

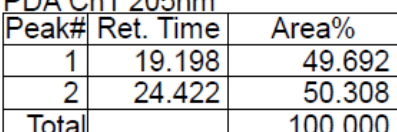

<Peak Table>

PDA Ch1 205nm

\begin{tabular}{|r|r|r|}
\hline Peak\# & Ret. Time & \multicolumn{1}{c|}{ Area\% } \\
\hline 1 & 19.065 & 96.841 \\
\hline 2 & 24.565 & 3.159 \\
\hline Total & & 100.000 \\
\hline
\end{tabular}


<smiles>COC1OCCC2OC1Oc1ccc(C)cc12</smiles>

(3aS,8aR)-5-methyl-2,3,3a,8a-tetrahydrofuro[2,3-b]benzofuran (3c)

Prepared according to GP2 using 2,3-dihydrofuran (1a) (76 $\mu \mathrm{L}, 1 \mathrm{mmol}$ ) and 2-bromo-4methylphenol (2c) $(24 \mu \mathrm{L}, 0.2 \mathrm{mmol})$. Isolated by column chromatography (Pentane: $\left.\mathrm{Et}_{2} \mathrm{O} 30: 1\right)$ as a pale yellow oil (68\% yield, 93\% ee) with $\mathrm{R}_{\mathrm{f}}=0.15 ;{ }^{1} \mathrm{H}-\mathrm{NMR}\left(\mathbf{C}_{6} \mathbf{D}_{6}, 400 \mathrm{MHz}\right): \delta(\mathrm{ppm})=1.48(\mathrm{~m}, 1 \mathrm{H}, \mathrm{H}-$ 3), $1.64\left(\mathrm{~m}, 1 \mathrm{H}, \mathrm{H}-3^{\prime}\right), 2.12(\mathrm{~s}, 3 \mathrm{H}, \mathrm{H}-13), 3.28\left(\mathrm{dd},{ }^{3} \mathrm{~J}_{\mathrm{H}-\mathrm{H}}=8.5 \mathrm{~Hz},{ }^{3} J_{\mathrm{H}-\mathrm{H}}=5.7 \mathrm{~Hz}, 1 \mathrm{H}, \mathrm{H}-4\right), 3.43\left(\mathrm{ddd},{ }^{2} J_{\mathrm{H}-}\right.$ $\left.{ }_{H}=11.9 \mathrm{~Hz},{ }^{3} J_{\mathrm{H}-\mathrm{H}}=8.6 \mathrm{~Hz},{ }^{3} \mathrm{~J}_{\mathrm{H}-\mathrm{H}}=4.9 \mathrm{~Hz}, 1 \mathrm{H}, \mathrm{H}-2\right), 3.65\left(\mathrm{t},{ }^{3} \mathrm{~J}_{\mathrm{H}-\mathrm{H}}=8.1 \mathrm{~Hz}, 1 \mathrm{H}, \mathrm{H}-2^{\prime}\right), 6.12\left(\mathrm{~d},{ }^{3} \mathrm{~J}_{\mathrm{H}-\mathrm{H}}=5.7\right.$ $\mathrm{Hz}, 1 \mathrm{H}, \mathrm{H}-12), 6.63(\mathrm{~m}, 1 \mathrm{H}, \mathrm{H}-6), 6.75(\mathrm{~m}, 2 \mathrm{H}, \mathrm{H}-8$ or $\mathrm{H}-9) ;{ }^{13} \mathbf{C}\left\{{ }^{1} \mathbf{H}\right\}-N M R\left(\mathbf{C}_{6} \mathbf{D}_{6}, 100 \mathrm{MHz}\right): \delta(\mathrm{ppm})=$ 20.8 (C-13), 33.7 (C-3), 46.8 (C-4), 66.9 (C-2), 109.0 (C-8 or C-9), 111.4 (C-12), 123.5 (C-8 or C-9),128.0 (C-5), 129.4 (C-6), 130.0 (C-7), 158.4 (C-10); GC-MS (EI): $\left(\mathrm{C}_{11} \mathrm{H}_{12} \mathrm{O}_{2}\right), 176.0\left(100, \mathrm{M}^{+}\right), 161.0\left(32, \mathrm{M}^{+}-\right.$ 15), $147\left(27, M^{+}-29\right), 91\left(42, M^{+}-85\right)$; IR spectrum (neat) $\left(\mathrm{cm}^{-1}\right)=2931,2975,1613,1487,1448$, $1306,1245,1201,1072,955,830,807,742,652 ;[\alpha]^{23}=-172.0\left(c 0.85, \mathrm{CH}_{2} \mathrm{Cl}_{2}\right)$; HPLC: OD-H, 206 $\mathrm{nm}$, Hexane: $\mathrm{PrOH}, 98: 2,1 \mathrm{~mL} / \mathrm{min}, 30^{\circ} \mathrm{C}, t_{\mathrm{R} 1}=7.8$ and $t_{\mathrm{R} 2}=8.2 \mathrm{~min}$.
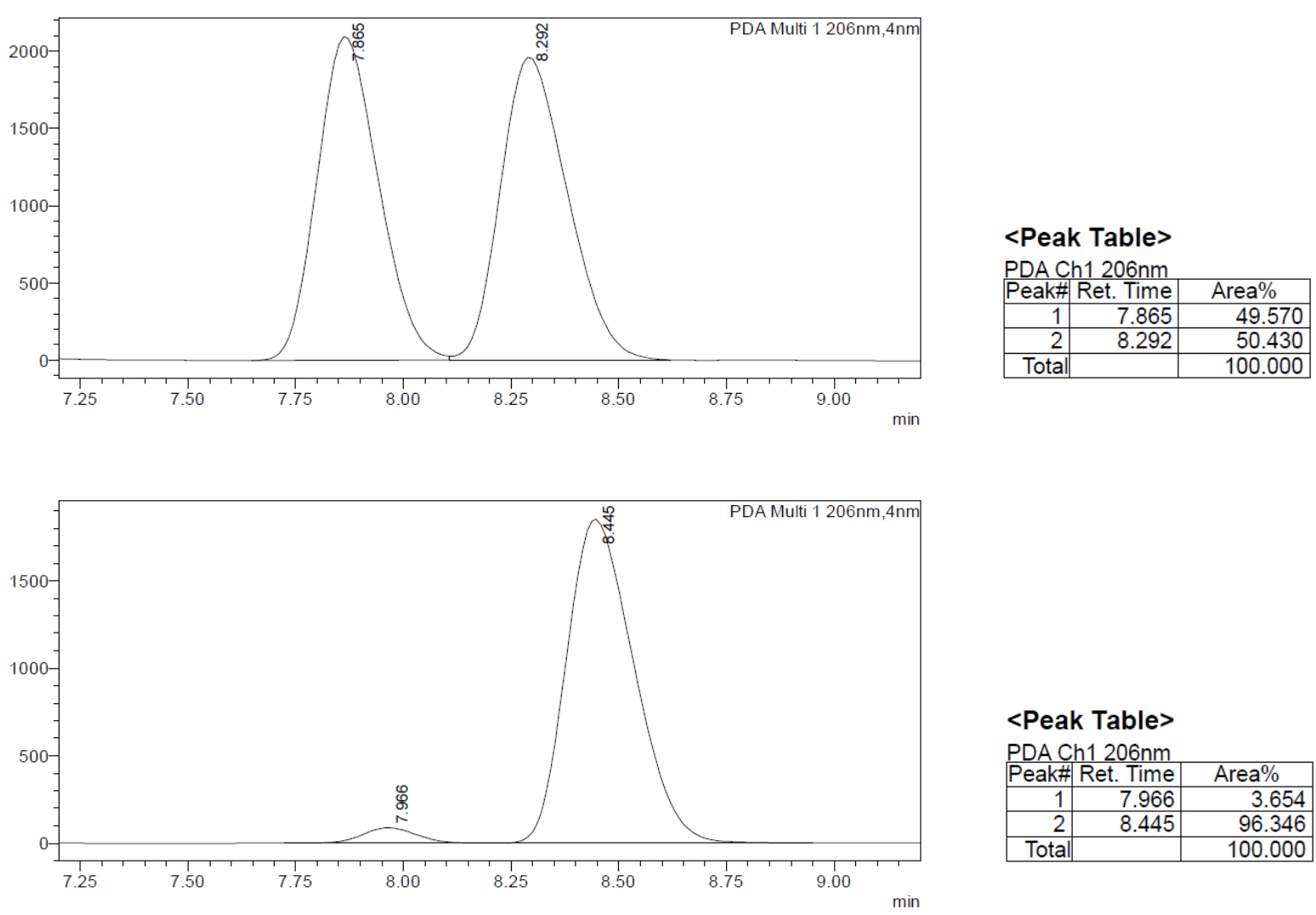
<smiles>COc1cccc2c1O[C@H]1CCO[C@H]2O1</smiles>

\section{(3aS,8aR)-7-Methoxy-2,3,3a,8a-tetrahydrofuro[2,3-b]benzofuran (3d)}

Prepared according to GP2 using 2,3-dihydrofuran (1a) (76 $\mu \mathrm{L}, 1 \mathrm{mmol}$ ) and 2-bromo-6methoxyphenol (2d) $(40.6 \mathrm{mg}, 0.2 \mathrm{mmol})$. Isolated by column chromatography (Pentane: $\mathrm{Et}_{2} \mathrm{O} 30: 1$ ) as a colorless oil (89\% yield, 88\% ee) with $\mathrm{R}_{\mathrm{f}}=0.1$ (Pentane:Et $\left.{ }_{2} \mathrm{O} 9: 1\right) ;{ }^{1} \mathrm{H}-\mathbf{N M R}\left(\mathbf{C}_{6} \mathrm{D}_{6}, 400 \mathrm{MHz}\right): \delta$ $(\mathrm{ppm})=1.47\left(\mathrm{dd},{ }^{2} J_{\mathrm{H}-\mathrm{H}}=12.0 \mathrm{~Hz},{ }^{3} J_{\mathrm{H}-\mathrm{H}}=4.9 \mathrm{~Hz}, 1 \mathrm{H}, \mathrm{H}-3\right), 1.55-1.67\left(\mathrm{~m}, 1 \mathrm{H}, \mathrm{H}-3^{\prime}\right), 3.29-3.34(\mathrm{~m}, 1 \mathrm{H}, \mathrm{H}-$ 4), $3.37\left(\mathrm{ddd},{ }^{3} \mathrm{~J}_{\mathrm{H}-\mathrm{H}}=9.7 \mathrm{~Hz},{ }^{3} \mathrm{~J}_{\mathrm{H}-\mathrm{H}}=7.5 \mathrm{~Hz},{ }^{4} J_{\mathrm{H}-\mathrm{H}}=3.8 \mathrm{~Hz}, 1 \mathrm{H}, \mathrm{H}-2\right), 3.47(\mathrm{~s}, 3 \mathrm{H}, \mathrm{H}-13), 3.61\left(\mathrm{t},{ }^{3} \mathrm{~J}_{\mathrm{H}-\mathrm{H}}=8.1\right.$ $\left.\mathrm{Hz}, 1 \mathrm{H}, \mathrm{H}-2^{\prime}\right), 6.11\left(\mathrm{~d},{ }^{3} \mathrm{~J}_{\mathrm{H}-\mathrm{H}}=5.7 \mathrm{~Hz}, 1 \mathrm{H}, \mathrm{H}-12\right), 6.56\left(\mathrm{dd},{ }^{3} \mathrm{~J}_{\mathrm{H}-\mathrm{H}}=7.5 \mathrm{~Hz},{ }^{4} J_{\mathrm{H}-\mathrm{H}}=0.6 \mathrm{~Hz}, 1 \mathrm{H}, \mathrm{H}-6\right), 6.58(\mathrm{~d}$, $\left.{ }^{3} \mathrm{~J}_{\mathrm{H}-\mathrm{H}}=8.1 \mathrm{~Hz}, 1 \mathrm{H}, \mathrm{H}-8\right), 6.75\left(\mathrm{t},{ }^{3} \mathrm{~J}_{\mathrm{H}-\mathrm{H}}=7.8 \mathrm{~Hz}, 1 \mathrm{H}, \mathrm{H}-7\right) ;{ }^{13} \mathrm{C}\left\{{ }^{1} \mathrm{H}\right\}-\mathrm{NMR}\left(\mathrm{C}_{6} \mathbf{D}_{6}, 100 \mathrm{MHz}\right): \delta(\mathrm{ppm})=33.6$ (C-3), 47.2 (C-4), 56.0 (C-13), 67.0 (C-2), 111.6 (C-12), 113.3 (C-8), 117.0 (C-6), 121.7 (C-7), 129.6 (C5), 144.5 (C-9), 149.1 (C-10); GC-MS (EI): $\left(\mathrm{C}_{11} \mathrm{H}_{12} \mathrm{O}_{3}\right), 192.1$ (100, $\left.\mathrm{M}^{+}\right), 177.1$ (26, $\left.\mathrm{M}^{+}-15\right), 149.1$ (40, $\left.M^{+}-43\right), 131.1\left(144, M^{+}-61\right), 103\left(23, M^{+}-89\right), 91.1\left(28, M^{+}-101\right), 77.1\left(19, M^{+}-115\right), 65\left(21, M^{+}\right.$ - 127); IR spectrum (neat) $\left(\mathrm{cm}^{-1}\right)=2946,1619,1594,1490,1461,1270,1062,930,732 ;[\alpha]^{23}=-$ 108.0 ( c 0.81, $\mathrm{CH}_{2} \mathrm{Cl}_{2}$ ); HPLC: AD-H, $207 \mathrm{~nm}$, Hexane: $\mathrm{PrOH}, 98: 2,1 \mathrm{~mL} / \mathrm{min}, 30^{\circ} \mathrm{C}, t_{\mathrm{R} 1}=10.8$ and $t_{\mathrm{R} 2}=$ $11.8 \mathrm{~min}$.
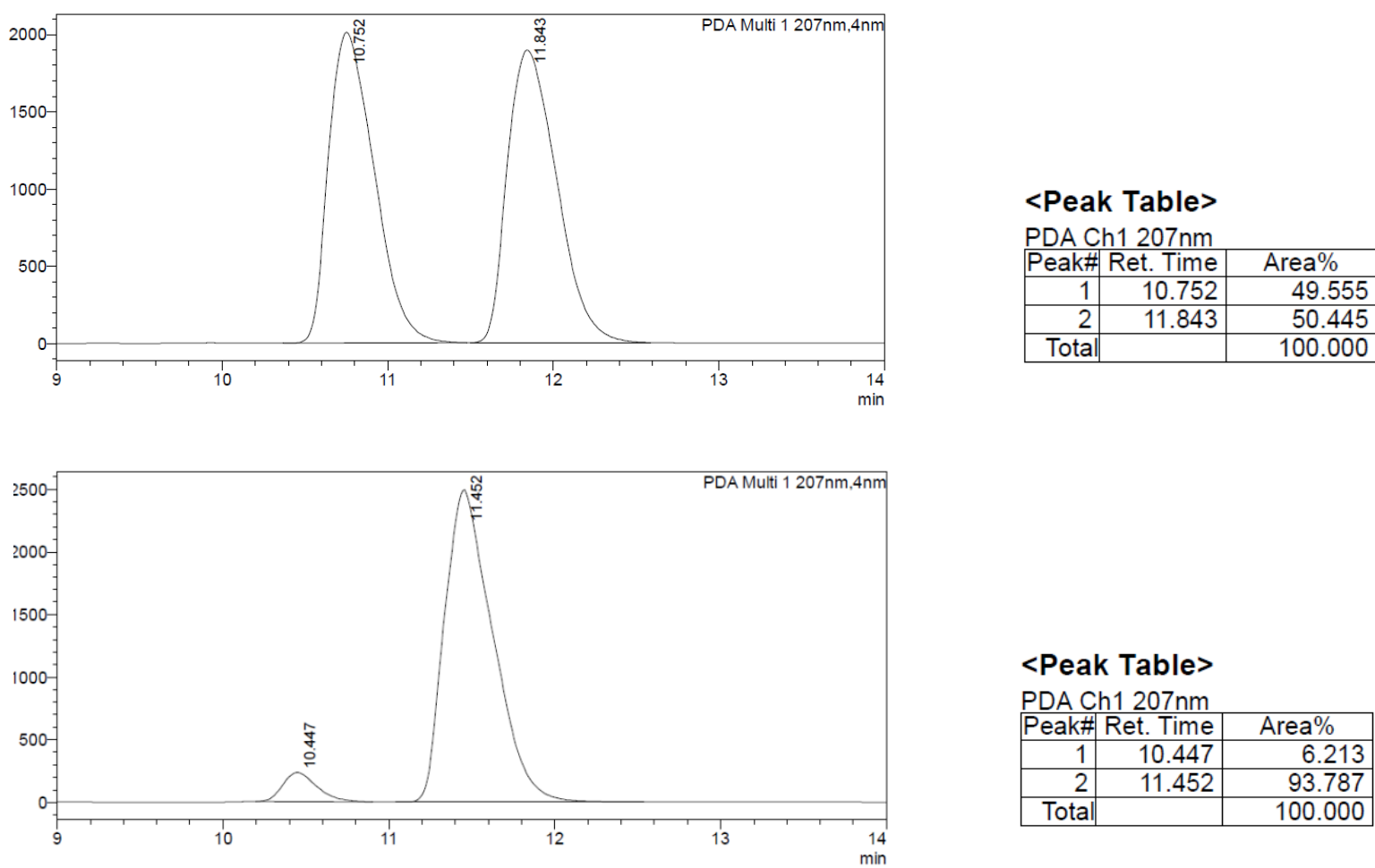

<Peak Table>

PDA Ch1 $207 \mathrm{~nm}$

\begin{tabular}{|r|r|r|}
\hline Peak\# & Ret. Time & \multicolumn{1}{|c|}{ Area\% } \\
\hline 1 & 10.447 & 6.213 \\
\hline 2 & 11.452 & 93.787 \\
\hline Total & & 100.000 \\
\hline
\end{tabular}


<smiles>COc1ccc2c(c1)OC1CCOCC1O2</smiles>

\section{(3aS,8aR)-6-Methoxy-2,3,3a,8a-tetrahydrofuro[2,3-b]benzofuran (3e)}

Prepared according to GP2 using 2,3-dihydrofuran (1a) (76 $\mu \mathrm{L}, 1 \mathrm{mmol}$ ) and 2-bromo-5methoxyphenol (2e) $(40.6 \mathrm{mg}, 0.2 \mathrm{mmol})$. Isolated by column chromatography (Pentane: $\mathrm{Et}_{2} \mathrm{O}$ 25:1) as a pale yellow oil (53\% yield, 90\% ee) with $\mathrm{R}_{\mathrm{f}}=0.10 ;{ }^{1} \mathbf{H}-\mathbf{N M R}\left(\mathbf{C}_{6} \mathbf{D}_{6}, 400 \mathbf{M H z}\right): \delta(\mathrm{ppm})=1.45(\mathrm{~m}$, $1 \mathrm{H}, \mathrm{H}-3), 1.62\left(\mathrm{~m}, 1 \mathrm{H}, \mathrm{H}-3^{\prime}\right), 3.26(\mathrm{~s}, 3 \mathrm{H}, \mathrm{H}-13), 3.29(\mathrm{~m}, 1 \mathrm{H}, \mathrm{H}-4), 3.43$ (ddd, ${ }^{2} J_{\mathrm{H}-\mathrm{H}}=12.0 \mathrm{~Hz},{ }^{3} J_{\mathrm{H}-\mathrm{H}}=8.6$ $\left.\mathrm{Hz},{ }^{3} J_{\mathrm{H}-\mathrm{H}}=4.9 \mathrm{~Hz}, 1 \mathrm{H}, \mathrm{H}-2\right), 3.66\left(\mathrm{t}^{3} \mathrm{~J}_{\mathrm{H}-\mathrm{H}}=8.0 \mathrm{~Hz}, 1 \mathrm{H}, \mathrm{H}-2^{\prime}\right), 6.14\left(\mathrm{~d},{ }^{3} \mathrm{~J}_{\mathrm{H}-\mathrm{H}}=5.6 \mathrm{~Hz}, 1 \mathrm{H}, \mathrm{H}-12\right), 6.48(\mathrm{dd}$, $\left.{ }^{3} J_{\mathrm{H}-\mathrm{H}}=8.0 \mathrm{~Hz},{ }^{4} J_{\mathrm{H}-\mathrm{H}}=2.3 \mathrm{~Hz}, 1 \mathrm{H}, \mathrm{H}-7\right), 6.51\left(\mathrm{~d},{ }^{4} J_{\mathrm{H}-\mathrm{H}}=2.3 \mathrm{~Hz}, 1 \mathrm{H}, \mathrm{H}-9\right), 6.73\left(\mathrm{~d},{ }^{3} J_{\mathrm{H}-\mathrm{H}}=7.3 \mathrm{~Hz}, 1 \mathrm{H}, \mathrm{H}-6\right)$; ${ }^{13} \mathrm{C}\left\{{ }^{1} \mathrm{H}\right\}-N M R\left(\mathrm{C}_{6} \mathrm{D}_{6}, 100 \mathrm{MHz}\right): \delta(\mathrm{ppm})=33.8$ (C-3), 46.2 (C-4), 54.9 (C-13), 67.0 (C-2), 95.6 (C-9). 107.6 (C-7), 112.3 (C-12), 120.0 (C-5), 124.9 (C-6), 161.5 (C-8 or C-10), 161.6 (C-8 or C-10); GC-MS (EI): $\left(\mathrm{C}_{11} \mathrm{H}_{12} \mathrm{O}_{3}\right), 192.1\left(100, \mathrm{M}^{+}\right), 177.0\left(26, \mathrm{M}^{+}-15\right), 163.1$ (48, $\left.\mathrm{M}^{+}-29\right), 135.1\left(20, \mathrm{M}^{+}-57\right), 103.1$ $\left(24, \mathrm{M}^{+}-89\right)$; IR spectrum (neat) $\left(\mathrm{cm}^{-1}\right)=2937,1621,1497,1446,1341,1299,1278,1197,1165$, $1146,1114,1074,1029,949,917,825,797,730 ;[\alpha]_{D}^{23}=-95.3\left(c 0.65, \mathrm{CH}_{2} \mathrm{Cl}_{2}\right) ;$ HPLC: OD-H, 208 $\mathrm{nm}$, Hexane: $\mathrm{PrOH}, 98: 2,1 \mathrm{~mL} / \mathrm{min}, 30^{\circ} \mathrm{C}, t_{\mathrm{R} 1}=10.8$ and $t_{\mathrm{R} 2}=11.9 \mathrm{~min}$.
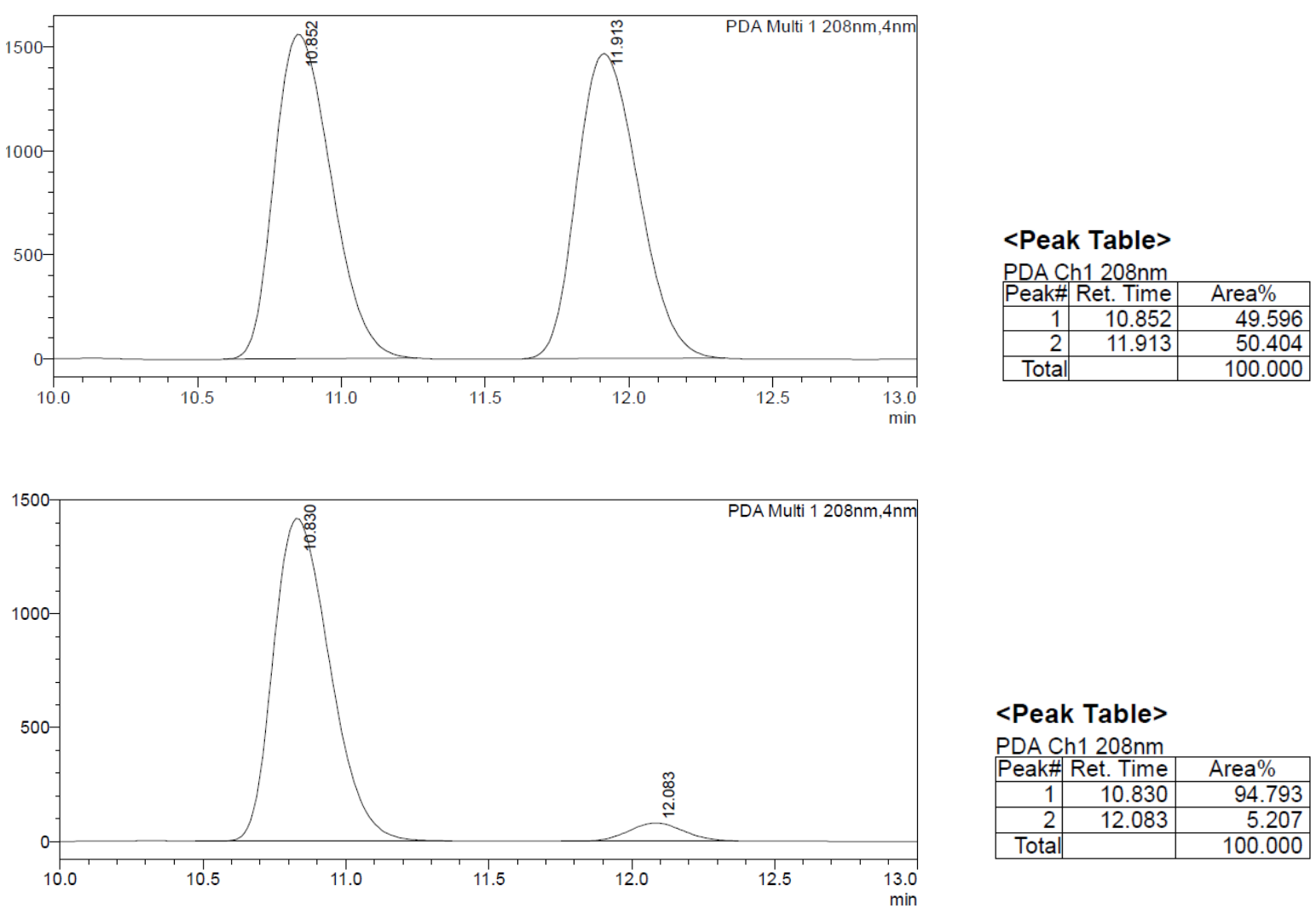
<smiles>COc1cccc2c1C1CCOC1OC2OC</smiles>

\section{(3aS,8aR)-4-Methoxy-2,3,3a,8a-tetrahydrofuro[2,3-b]benzofuran (3f)}

Prepared according to GP2 using 2,3-dihydrofuran (1a) (76 $\mu \mathrm{L}, 1 \mathrm{mmol}$ ) and 2-bromo-3methoxyphenol (2f) $(40.6 \mathrm{mg}, 0.2 \mathrm{mmol})$. Isolated by column chromatography (Pentane: $\mathrm{Et}_{2} \mathrm{O} 30: 1$ ) as a colorless oil ( $42 \%$ yield, $57 \%$ ee) with $\mathrm{R}_{\mathrm{f}}=0.2$ (Pentane:Et $\left.{ }_{2} \mathrm{O} 9: 1\right) ;{ }^{1} \mathbf{H}-\mathbf{N M R}\left(\mathbf{C}_{6} \mathbf{D}_{6}, 400 \mathbf{M H z}\right): \delta$ $(p p m)=1.72\left(\mathrm{ddt},{ }^{2} J_{\mathrm{H}-\mathrm{H}}=12.1 \mathrm{~Hz},{ }^{3} J_{\mathrm{H}-\mathrm{H}}=8.7 \mathrm{~Hz},{ }^{3} J_{\mathrm{H}-\mathrm{H}}=7.7 \mathrm{~Hz}, 1 \mathrm{H}, \mathrm{H}-3\right), 1.94\left(\mathrm{ddd},{ }^{2} J_{\mathrm{H}-\mathrm{H}}=12.1 \mathrm{~Hz},{ }^{3} J_{\mathrm{H}-\mathrm{H}}\right.$ $\left.=5.0 \mathrm{~Hz},{ }^{4} J_{\mathrm{H}-\mathrm{H}}=0.9 \mathrm{~Hz}, 1 \mathrm{H}, \mathrm{H}-3^{\prime}\right), 3.31(\mathrm{~s}, 3 \mathrm{H}, \mathrm{H}-13), 3.46$ (ddd, ${ }^{2} J_{\mathrm{H}-\mathrm{H}}=12.1 \mathrm{~Hz},{ }^{3} J_{\mathrm{H}-\mathrm{H}}=8.7 \mathrm{~Hz},{ }^{3} J_{\mathrm{H}-\mathrm{H}}=5.0$ $\mathrm{Hz}, 1 \mathrm{H}, \mathrm{H}-2), 3.62\left(\mathrm{dd},{ }^{3} J_{\mathrm{H}-\mathrm{H}}=8.7 \mathrm{~Hz},{ }^{3} \mathrm{~J}_{\mathrm{H}-\mathrm{H}}=5.7 \mathrm{~Hz}, 1 \mathrm{H}, \mathrm{H}-4\right), 3.68\left(\mathrm{ddd},{ }^{3} \mathrm{~J}_{\mathrm{H}-\mathrm{H}}=8.5 \mathrm{~Hz},{ }^{3} \mathrm{~J}_{\mathrm{H}-\mathrm{H}}=7.7 \mathrm{~Hz},{ }^{4} J_{\mathrm{H}-}\right.$ $\left.\mathrm{H}=0.8 \mathrm{~Hz}, 1 \mathrm{H}, \mathrm{H}-2^{\prime}\right), 6.12\left(\mathrm{~d}^{3} \mathrm{~J}_{\mathrm{H}-\mathrm{H}}=8.2 \mathrm{~Hz}, 1 \mathrm{H}, \mathrm{H}-7\right), 6.13\left(\mathrm{~d},{ }^{3} \mathrm{~J}_{\mathrm{H}-\mathrm{H}}=5.7 \mathrm{~Hz}, 1 \mathrm{H}, \mathrm{H}-12\right), 6.59\left(\mathrm{~d},{ }^{3} J_{\mathrm{H}-\mathrm{H}}=\right.$ $8.0 \mathrm{~Hz}, 1 \mathrm{H}, \mathrm{H}-9), 6.94\left(\mathrm{dt},{ }^{3} \mathrm{~J}_{\mathrm{H}-\mathrm{H}}=8.2 \mathrm{~Hz},{ }^{4} J_{\mathrm{H}-\mathrm{H}}=0.8 \mathrm{~Hz}, 1 \mathrm{H}, \mathrm{H}-8\right) ;{ }^{13} \mathrm{C}\left\{{ }^{1} \mathbf{H}\right\}-\mathrm{NMR}\left(\mathrm{C}_{6} \mathbf{D}_{6}, 100 \mathrm{MHz}\right): \delta$ $(\mathrm{ppm})=31.8$ (C-3), 45.2 (C-4), 54.9 (C-13), 67.1 (C-2), 102.9 (C-9), 103.3 (C-7), 111.9 (C-12), 114.9 (C5), 130.0 (C-8), 156.9 (C-6), 161.6 (C-10); GC-MS (EI): $\left(\mathrm{C}_{11} \mathrm{H}_{12} \mathrm{O}_{3}\right), 192.1\left(100, \mathrm{M}^{+}\right), 177$ (49, $\left.\mathrm{M}^{+}-15\right)$, $163.1\left(12, M^{+}-29\right), 148.9\left(34, M^{+}-43\right), 135\left(19, M^{+}-57\right), 121\left(16, M^{+}-71\right), 107\left(16, M^{+}-85\right)$, 103.1 (17, $\left.\mathrm{M}^{+}-89\right)$, 91.1 (19, $\left.\mathrm{M}^{+}-101\right), 85.1\left(12, \mathrm{M}^{+}-107\right), 77.1$ (31, $\left.\mathrm{M}^{+}-115\right), 65.1\left(21, \mathrm{M}^{+}-127\right)$; IR spectrum (neat) $\left(\mathrm{cm}^{-1}\right)=2956,1604,1461,1269,1239,1076,951,931,768 ;[\alpha]^{23}{ }_{\mathrm{D}}=-102.2(\mathrm{c}$ 0.36, $\mathrm{CH}_{2} \mathrm{Cl}_{2}$ ); HPLC: AD-H, $208 \mathrm{~nm}$, Hexane: $: \mathrm{PrOH}, 98: 2,1 \mathrm{~mL} / \mathrm{min}, 30^{\circ} \mathrm{C}, t_{\mathrm{R} 1}=8.6$ and $t_{\mathrm{R} 2}=9.2 \mathrm{~min}$.
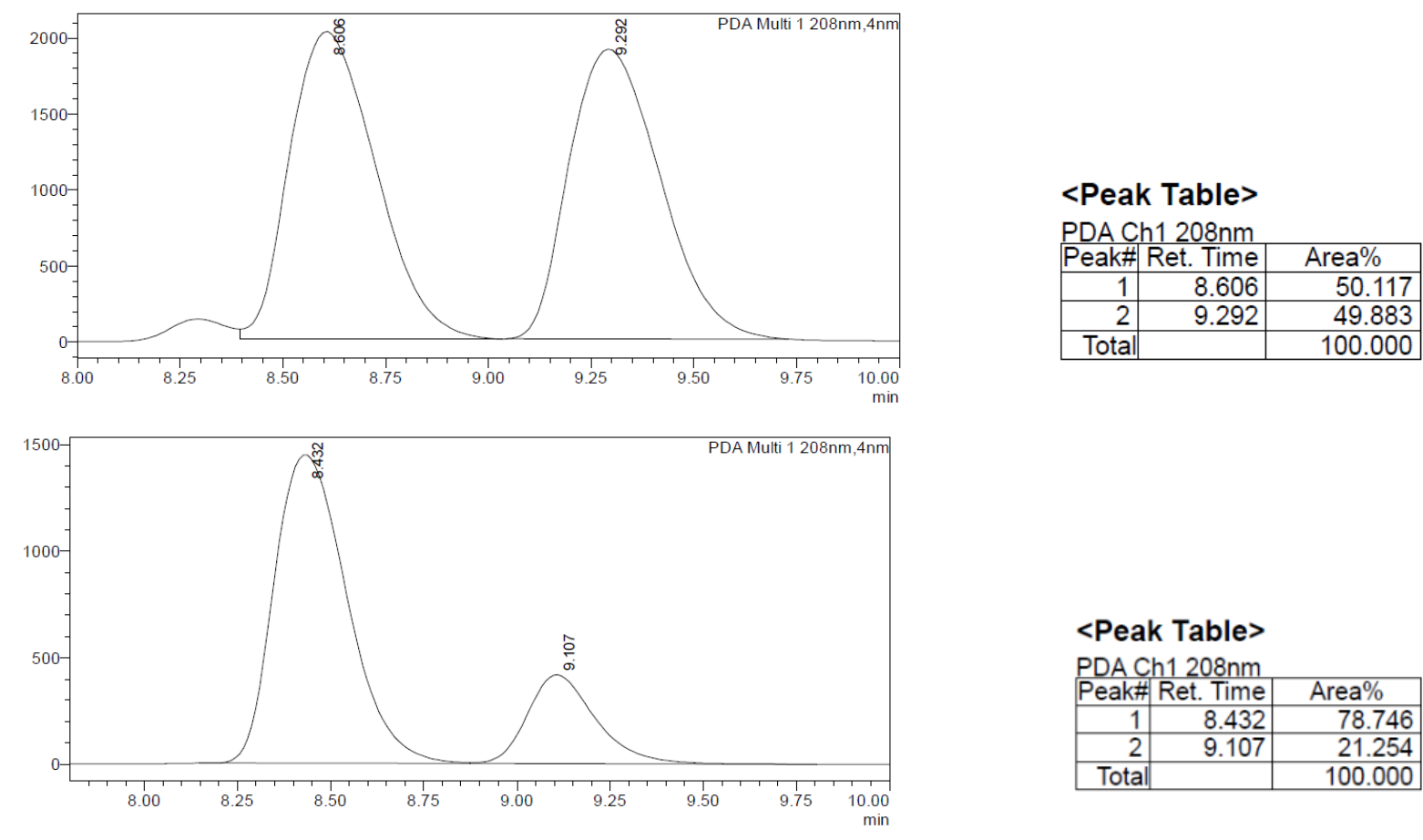
<smiles>COC1Oc2cc(C)ccc2[C@H]2CCO[C@H]12</smiles>

(3aS,8aR)-6-Methyl-2,3,3a,8a-tetrahydrofuro[2,3-b]benzofuran (3g)

Prepared according to GP2 using 2,3-dihydrofuran (1a) (76 $\mu \mathrm{L}, 1 \mathrm{mmol}$ ) and 2-bromo-3methylphenol (2g) (37.4 mg, $0.2 \mathrm{mmol})$. Isolated by column chromatography (Pentane:Et $\left.{ }_{2} \mathrm{O} 30: 1\right)$ as a white solid (20 mg, 57\% yield, $97 \%$ ee) with $\mathrm{R}_{\mathrm{f}}=0.2$ (Pentane: $\mathrm{Et}_{2} \mathrm{O} 9: 1$ ); $\mathrm{mp}=46{ }^{\circ} \mathrm{C} ;{ }^{1} \mathbf{H}-\mathbf{N M R}\left(\mathbf{C}_{6} \mathbf{D}_{6}\right.$, $400 \mathrm{MHz}$ ): $1.46\left(\mathrm{dd}^{2}{ }^{2} \mathrm{~J}_{\mathrm{H}-\mathrm{H}}=12.1 \mathrm{~Hz},{ }^{3} J_{\mathrm{H}-\mathrm{H}}=4.9 \mathrm{~Hz}, 1 \mathrm{H}, \mathrm{H}-3\right), 1.55-1.69\left(\mathrm{~m}, 1 \mathrm{H}, \mathrm{H}-3^{\prime}\right), 2.07(\mathrm{~s}, 3 \mathrm{H}, \mathrm{H}-13)$, $3.29\left(\mathrm{t},{ }^{3} \mathrm{~J}_{\mathrm{H}-\mathrm{H}}=6.8 \mathrm{~Hz}, 1 \mathrm{H}, \mathrm{H}-4\right), 3.40\left(\mathrm{ddd},{ }^{2} \mathrm{~J}_{\mathrm{H}-\mathrm{H}}=11.9 \mathrm{~Hz},{ }^{3} \mathrm{~J}_{\mathrm{H}-\mathrm{H}}=8.7 \mathrm{~Hz},{ }^{3} \mathrm{~J}_{\mathrm{H}-\mathrm{H}}=5.0 \mathrm{~Hz}, 1 \mathrm{H}, \mathrm{H}-2\right), 3.65$ $\left(\mathrm{dt},{ }^{3} J_{\mathrm{H}-\mathrm{H}}=8.1 \mathrm{~Hz},{ }^{4} J_{\mathrm{H}-\mathrm{H}}=1.7 \mathrm{~Hz}, 1 \mathrm{H}, \mathrm{H}-2^{\prime}\right), 6.12\left(\mathrm{dd},{ }^{3} J_{\mathrm{H}-\mathrm{H}}=5.7 \mathrm{~Hz},{ }^{4} J_{\mathrm{H}-\mathrm{H}}=1.8 \mathrm{~Hz}, 1 \mathrm{H}, \mathrm{H}-12\right), 6.62\left(\mathrm{~d},{ }^{3} J_{\mathrm{H}-\mathrm{H}}\right.$ $\left.=7.5 \mathrm{~Hz}, 1 \mathrm{H}, \mathrm{H}-7), 6.69(\mathrm{~s}, 1 \mathrm{H}, \mathrm{H}-9), 6.77\left(\mathrm{~d},{ }^{3} \mathrm{~J}_{\mathrm{H}-\mathrm{H}}=6.9 \mathrm{~Hz}, 1 \mathrm{H}, \mathrm{H}-6\right) ;{ }^{13} \mathrm{C}^{1}{ }^{\mathrm{H}}\right\}-\mathrm{NMR}\left(\mathrm{C}_{6} \mathrm{D}_{6}, 100 \mathrm{MHz}\right):$ 21.5 (C-13), 33.8 (C-3), 46.5 (C-4), 66.9 (C-2), 110.2 (C-9), 111.7 (C-12), 121.9 (C-7), 124.4 (C-6), 125.4 (C-5), 139.0 (C-8), 160.7 (C-10); GC-MS (EI): $\left(\mathrm{C}_{11} \mathrm{H}_{12} \mathrm{O}_{2}\right), 176.0\left(100, \mathrm{M}^{+}\right), 161.0\left(65, \mathrm{M}^{+}-15\right), 145.1$ (34, $\left.\mathrm{M}^{+}-31\right), 133.1\left(32, \mathrm{M}^{+}-43\right), 115.0\left(32, \mathrm{M}^{+}-61\right), 91.1\left(42, \mathrm{M}^{+}-85\right), 64.9\left(26, \mathrm{M}^{+}-111\right), 56.1$ $\left(31, \mathrm{M}^{+}-120\right)$; IR spectrum (neat) $\left(\mathrm{cm}^{-1}\right)=2927,1592,1498,1454,1251,1112,1069,946,922,801$, 750; $[\alpha]^{23}{ }_{\mathrm{D}}=-123.9\left(\mathrm{c} 0.67, \mathrm{CH}_{2} \mathrm{Cl}_{2}\right.$ ); HPLC: IC, $206 \mathrm{~nm}$, Hexane: $\mathrm{PrOH}, 98: 2,1 \mathrm{~mL} / \mathrm{min}, 30^{\circ} \mathrm{C}, t_{\mathrm{R} 1}=$ 10.3 and $t_{\mathrm{R} 2}=14.4 \mathrm{~min}$.
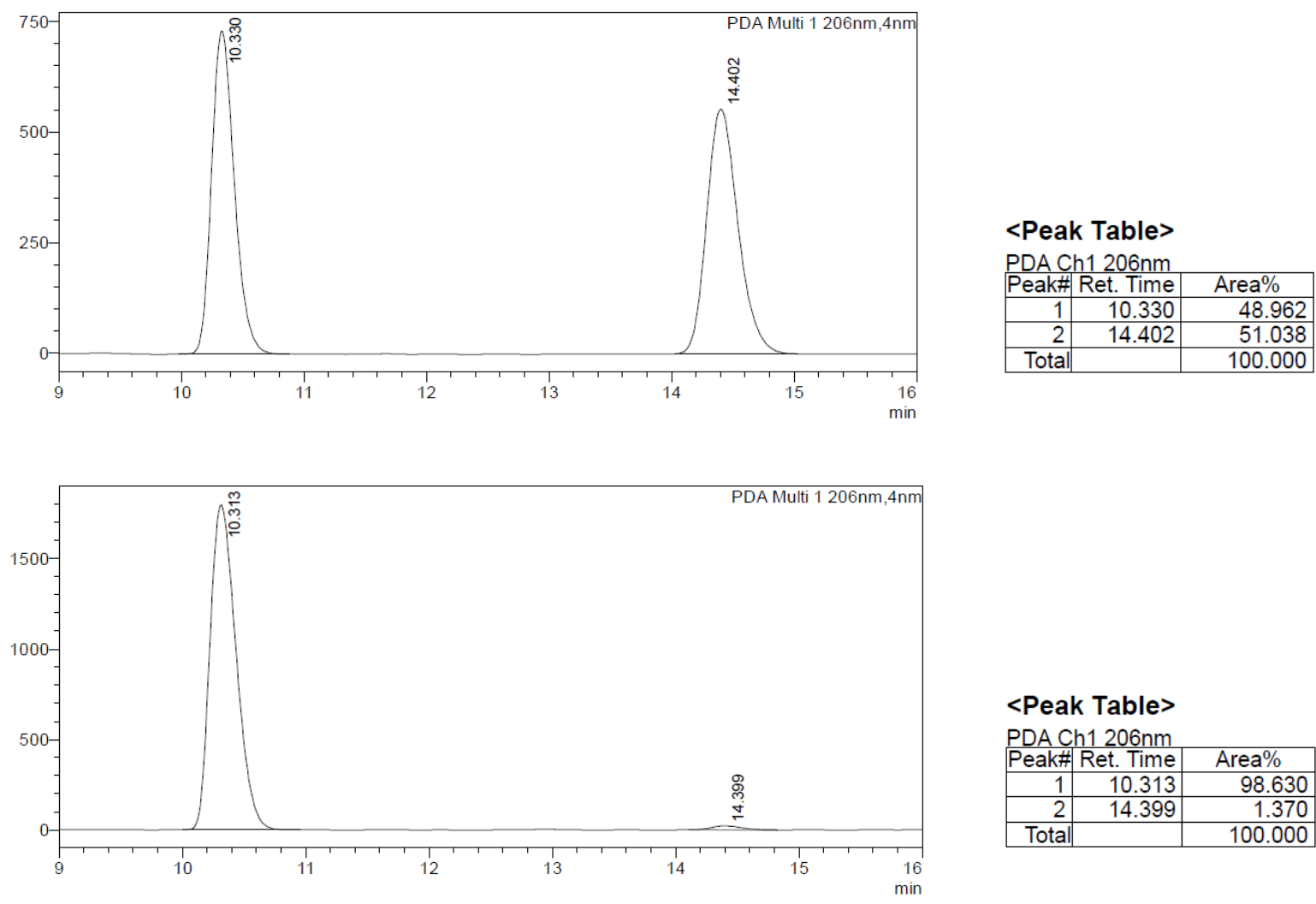
<smiles>Fc1cc(F)c2c(c1)C1CCOC1OCCO2</smiles>

\section{(3aS,8aR)-5,7-Difluoro-2,3,3a,8a-tetrahydrofuro[2,3-b]benzofuran (3h)}

Prepared according to GP2 using 2,3-dihydrofuran (1a) (76 $\mu \mathrm{L}, 1 \mathrm{mmol}$ ) and 2-bromo-4,6difluorophenol ( $2 \mathrm{~h})(23 \mu \mathrm{L}, 0.2 \mathrm{mmol})$. Isolated by column chromatography (Pentane: $\left.\mathrm{Et}_{2} \mathrm{O} 30: 1\right)$ as a white solid (91\% yield, 94\% ee) with $\mathrm{R}_{\mathrm{f}}=0.1$ (Pentane:Et $\mathrm{E}_{2} \mathrm{O} 9: 1$ ); $\mathrm{mp}=67^{\circ} \mathrm{C} ;{ }^{1} \mathbf{H}-\mathbf{N M R}\left(\mathbf{C}_{6} \mathrm{D}_{6}, 400\right.$ $\mathrm{MHz}): \delta(\mathrm{ppm})=1.16\left(\mathrm{dd},{ }^{2} \mathrm{H}_{\mathrm{H}-\mathrm{H}}=12.3 \mathrm{~Hz},{ }^{3} J_{\mathrm{H}-\mathrm{H}}=4.9 \mathrm{~Hz}, 1 \mathrm{H}, \mathrm{H}-3\right), 1.36-1.47\left(\mathrm{~m}, 1 \mathrm{H}, \mathrm{H}-3^{\prime}\right), 2.99\left(\mathrm{dd},{ }^{3} \mathrm{~J}_{\mathrm{H}}\right.$ $\left.{ }_{H}=8.5 \mathrm{~Hz},{ }^{3} J_{H-H}=5.8 \mathrm{~Hz}, 1 \mathrm{H}, \mathrm{H}-4\right), 3.18\left(\mathrm{ddd},{ }^{2} J_{\mathrm{H}-\mathrm{H}}=12.2 \mathrm{~Hz},{ }^{3} J_{\mathrm{H}-\mathrm{H}}=8.8 \mathrm{~Hz},{ }^{3} \mathrm{~J}_{\mathrm{H}-\mathrm{H}}=5.0 \mathrm{~Hz}, 1 \mathrm{H}, \mathrm{H}-2\right), 3.54$ $\left(\mathrm{t},{ }^{3} J_{\mathrm{H}-\mathrm{H}}=8.2 \mathrm{~Hz}, 1 \mathrm{H}, \mathrm{H}-2^{\prime}\right), 5.87\left(\mathrm{~d}^{3}{ }^{3} \mathrm{H}_{\mathrm{H}-\mathrm{H}}=5.6 \mathrm{~Hz}, 1 \mathrm{H}, \mathrm{H}-12\right), 6.14-6.19(\mathrm{~m}, 1 \mathrm{H}, \mathrm{H}-6), 6.43\left(\mathrm{dt}^{3} \mathrm{~J}_{\mathrm{H}-\mathrm{F}}=9.7\right.$ $\left.\mathrm{Hz},{ }^{4} J_{\mathrm{H}-\mathrm{H}}=2.4 \mathrm{~Hz}, 1 \mathrm{H}, \mathrm{H}-8\right) ;{ }^{13} \mathrm{C}\left\{{ }^{1} \mathrm{H}\right\}-\mathrm{NMR}\left(\mathrm{C}_{6} \mathrm{D}_{6}, 100 \mathrm{MHz}\right): \delta(\mathrm{ppm})=33.1(\mathrm{C}-3), 47.2\left(\mathrm{t},{ }^{4} J_{\mathrm{C}-\mathrm{F}}=2.1 \mathrm{~Hz}\right.$, C-4), 67.1 (C-2), 104.1 (dd, ${ }^{2} J_{C-F}=27.6 \mathrm{~Hz},{ }^{2} J_{C-F}=21.0 \mathrm{~Hz}, \mathrm{C}-8$ ), $107.2\left(\mathrm{dd},{ }^{2} J_{\mathrm{C}-\mathrm{F}}=24.2 \mathrm{~Hz},{ }^{4} \mathrm{~J}_{\mathrm{C}-\mathrm{F}}=4.0 \mathrm{~Hz}\right.$, C-6), 112.8 (C-12), 132.0 (dd, ${ }^{2} J_{C-F}=9.6 \mathrm{~Hz},{ }^{4} J_{C-F}=4.1 \mathrm{~Hz}, \mathrm{C}-5$ ), 143.1 (dd, ${ }^{3} J_{C-F}=10.5 \mathrm{~Hz},{ }^{4} J_{C-F}=2.7 \mathrm{~Hz}$, C-10), $146.0\left(\mathrm{dd},{ }^{1} J_{\mathrm{C}-\mathrm{F}}=249.3 \mathrm{~Hz},{ }^{3} \mathrm{~J}_{\mathrm{C}-\mathrm{F}}=12.6 \mathrm{~Hz}, \mathrm{C}-9\right.$ ), $157.2\left(\mathrm{dd},{ }^{1} J_{\mathrm{C}-\mathrm{F}}=240.2 \mathrm{~Hz},{ }^{3} J_{\mathrm{C}-\mathrm{F}}=8.8 \mathrm{~Hz}, \mathrm{C}-7\right.$ ); ${ }^{19} \mathrm{~F}-\mathrm{NMR}\left(\mathrm{C}_{6} \mathbf{D}_{6}, 282 \mathrm{MHz}\right): \delta(\mathrm{ppm})=-119.7\left(\mathrm{~d},{ }^{3} \mathrm{~J}_{\mathrm{F}-\mathrm{F}}=6.8 \mathrm{~Hz}, 1 \mathrm{~F}\right),-132.9\left(\mathrm{~d},{ }^{3} \mathrm{~J}_{\mathrm{F}-\mathrm{F}}=6.7 \mathrm{~Hz}, 1 \mathrm{~F}\right)$; GC-MS (EI): $\left(\mathrm{C}_{10} \mathrm{H}_{8} \mathrm{~F}_{2} \mathrm{O}_{2}\right), 198.1\left(100, \mathrm{M}^{+}\right), 183.1$ (27, $\left.\mathrm{M}^{+}-15\right), 167.1$ (35, $\left.\mathrm{M}^{+}-31\right), 141$ (18, $\left.\mathrm{M}^{+}-57\right), 127.1$ (28, $\left.\mathrm{M}^{+}-71\right), 121.1\left(27, \mathrm{M}^{+}-77\right), 114\left(38, \mathrm{M}^{+}-84\right), 101.1\left(24, \mathrm{M}^{+}-97\right)$; IR spectrum (neat) $\left(\mathrm{cm}^{-1}\right)=$ 2888, 1636, 1488, 1470, 1072, 1051, 910, 871, 796; $[\alpha]^{23}=-101.9\left(c 0.56, \mathrm{CH}_{2} \mathrm{Cl}_{2}\right.$ ); HPLC: OJ-H, 205 $\mathrm{nm}$, Hexane: $\mathrm{PrOH}, 98: 2,1 \mathrm{~mL} / \mathrm{min}, 30^{\circ} \mathrm{C}, t_{\mathrm{R} 1}=13.4$ and $t_{\mathrm{R} 2}=14.4 \mathrm{~min}$.
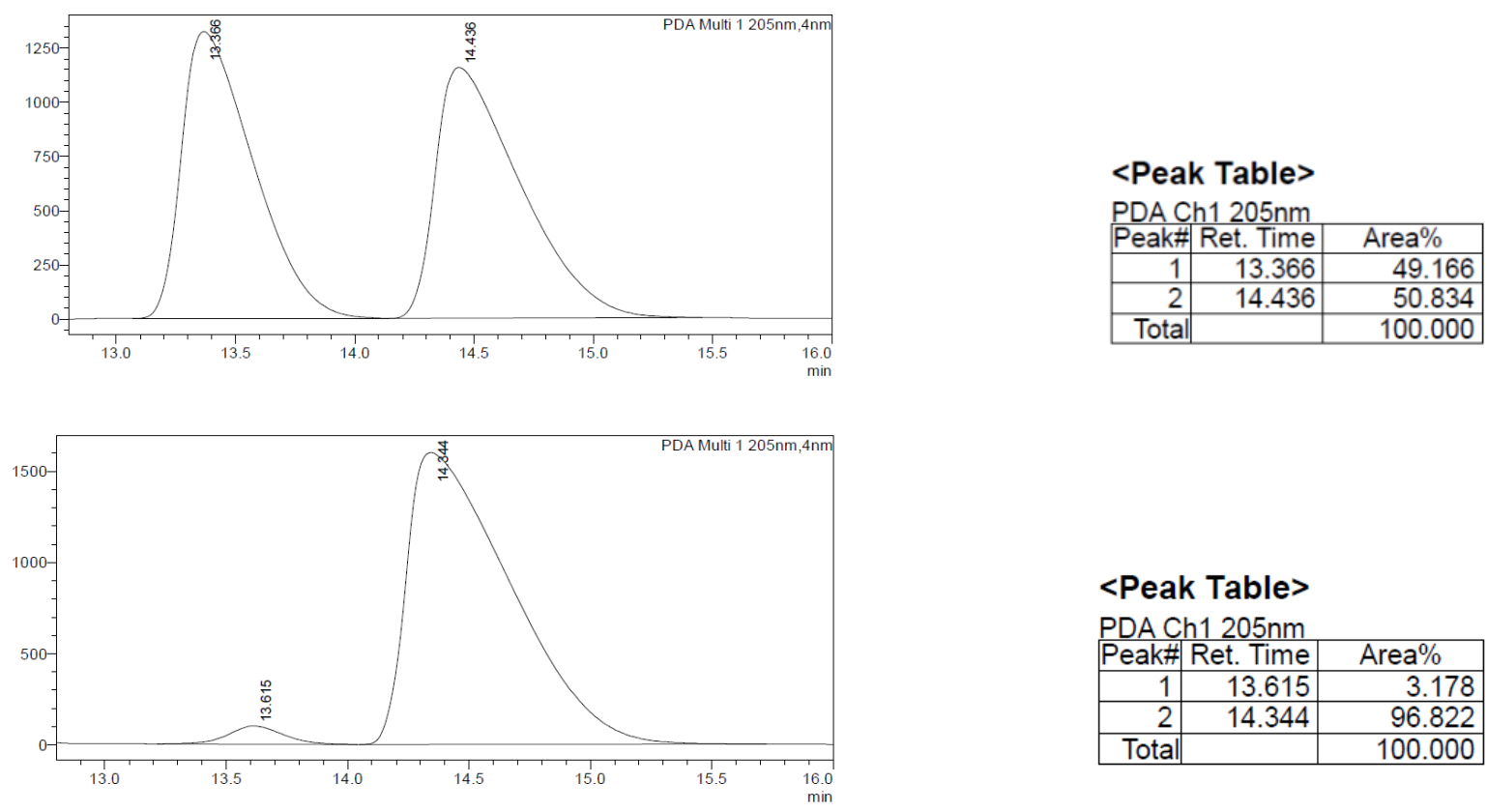

\begin{tabular}{|c|c|c|}
\hline \\
\hline \multicolumn{3}{|c|}{$\begin{array}{l}<\text { Peak Table> } \\
\text { PDA Ch1 205nm }\end{array}$} \\
\hline 1 & 13.615 & $\frac{a \%}{3.178}$ \\
\hline 2 & 14.344 & 96.822 \\
\hline Total & & 100.000 \\
\hline
\end{tabular}


<smiles>COC1Oc2cc(F)ccc2C1CCO</smiles>

\section{(3aS,8aR)-6-Fluoro-2,3,3a,8a-tetrahydrofuro[2,3-b]benzofuran (3i)}

Prepared according to GP2 using 2,3-dihydrofuran (1a) (76 $\mu \mathrm{L}, 1 \mathrm{mmol}$ ) and 2-bromo-5-fluorophenol (2i) $(38.2 \mathrm{mg}, 0.2 \mathrm{mmol})$. Isolated by column chromatography (Pentane: $\mathrm{Et}_{2} \mathrm{O} 30: 1$ ) as a pale yellow oil (58\% yield, 88\% ee) with $\mathrm{R}_{\mathrm{f}}=0.20 ;{ }^{1} \mathrm{H}-\mathrm{NMR}\left(\mathrm{C}_{6} \mathrm{D}_{6}, 400 \mathrm{MHz}\right): \delta(\mathrm{ppm})=1.30\left(\mathrm{~m}, 1 \mathrm{H}, \mathrm{H}-3^{\prime}\right), 1.50$ $(\mathrm{m}, 1 \mathrm{H}, \mathrm{H}-3), 3.09(\mathrm{~m}, 1 \mathrm{H}, \mathrm{H}-4), 3.27\left(\mathrm{ddd},{ }^{2} J_{\mathrm{H}-\mathrm{H}}=12.0 \mathrm{~Hz},{ }^{3} J_{\mathrm{H}-\mathrm{H}}=8.7 \mathrm{~Hz},{ }^{3} J_{\mathrm{H}-\mathrm{H}}=4.9 \mathrm{~Hz}, 1 \mathrm{H}, \mathrm{H}-2\right), 3.59(\mathrm{t}$, $\left.{ }^{3} J_{\mathrm{H}-\mathrm{H}}=8.2 \mathrm{~Hz}, 1 \mathrm{H}, \mathrm{H}-2^{\prime}\right), 6.00\left(\mathrm{~d}^{3} \mathrm{~J}_{\mathrm{H}-\mathrm{H}}=5.6 \mathrm{~Hz}, 1 \mathrm{H}, \mathrm{H}-12\right), 6.44(\mathrm{~m}, 1 \mathrm{H}, \mathrm{H}-6, \mathrm{H}-7$ or H-8), $6.53(\mathrm{~m}, 2 \mathrm{H}$, H-6, H-7 or H-8); $\left.{ }^{13} \mathrm{C}^{1}{ }^{1} \mathrm{H}\right\}-N M R\left(\mathrm{C}_{6} \mathrm{D}_{6}, 100 \mathrm{MHz}\right): \delta(\mathrm{ppm})=33.6$ (C-3), 45.9 (C-4), 66.9 (C-2), 97.8 (d, ${ }^{2} J_{\mathrm{C}-\mathrm{F}}=26.4 \mathrm{~Hz}, \mathrm{C}-7$ or C-8), 107.7 (d, ${ }^{2} \mathrm{~J}_{\mathrm{C}-\mathrm{F}}=22.8 \mathrm{~Hz}, \mathrm{C}-7$ or C-8), $112.6(\mathrm{C}-12), 123.9$ (d, ${ }^{4} \mathrm{~J}_{\mathrm{C}-\mathrm{F}}=2.6 \mathrm{~Hz}, \mathrm{C}-$ 5), $125.1\left(\mathrm{~d},{ }^{3} \mathrm{~J}_{\mathrm{C}-\mathrm{F}}=10.4 \mathrm{~Hz}, \mathrm{C}-6\right), 161.3\left(\mathrm{~d},{ }^{3} \mathrm{~J}_{\mathrm{C}-\mathrm{F}}=12.8 \mathrm{~Hz}, \mathrm{C}-10\right), 163.9\left(\mathrm{~d},{ }^{1} J_{\mathrm{C}-\mathrm{F}}=243.8 \mathrm{~Hz}, \mathrm{C}-8\right) ;{ }^{19} \mathrm{~F}-$ NMR ( $\left.\mathbf{C}_{6} \mathbf{D}_{6}, 282 \mathrm{MHz}\right): \delta(\mathrm{ppm})=-111.1(\mathrm{~s}, 1 \mathrm{~F})$; GC-MS (EI): $\left(\mathrm{C}_{10} \mathrm{H}_{9} \mathrm{FO}_{2}\right), 180.1\left(100, \mathrm{M}^{+}\right), 165.0(44$, $\left.M^{+}-15\right), 149.0\left(33, M^{+}-31\right), 109.0\left(23, M^{+}-71\right), 103.1\left(31, M^{+}-77\right)$; IR spectrum (neat) $\left(\mathrm{cm}^{-1}\right)=$ $2880,1611,1492,1439,1326,1260,1158,1133,1073,951,917,837,799,731 ;[\alpha]_{D}^{23}=-126.5(c$ 0.66, $\mathrm{CH}_{2} \mathrm{Cl}_{2}$ ); HPLC: IC, $205 \mathrm{~nm}$, Hexane: $: \mathrm{PrOH}, 98: 2,1 \mathrm{~mL} / \mathrm{min}, 30^{\circ} \mathrm{C}, t_{\mathrm{R} 1}=8.4$ and $t_{\mathrm{R} 2}=9.7 \mathrm{~min}$.
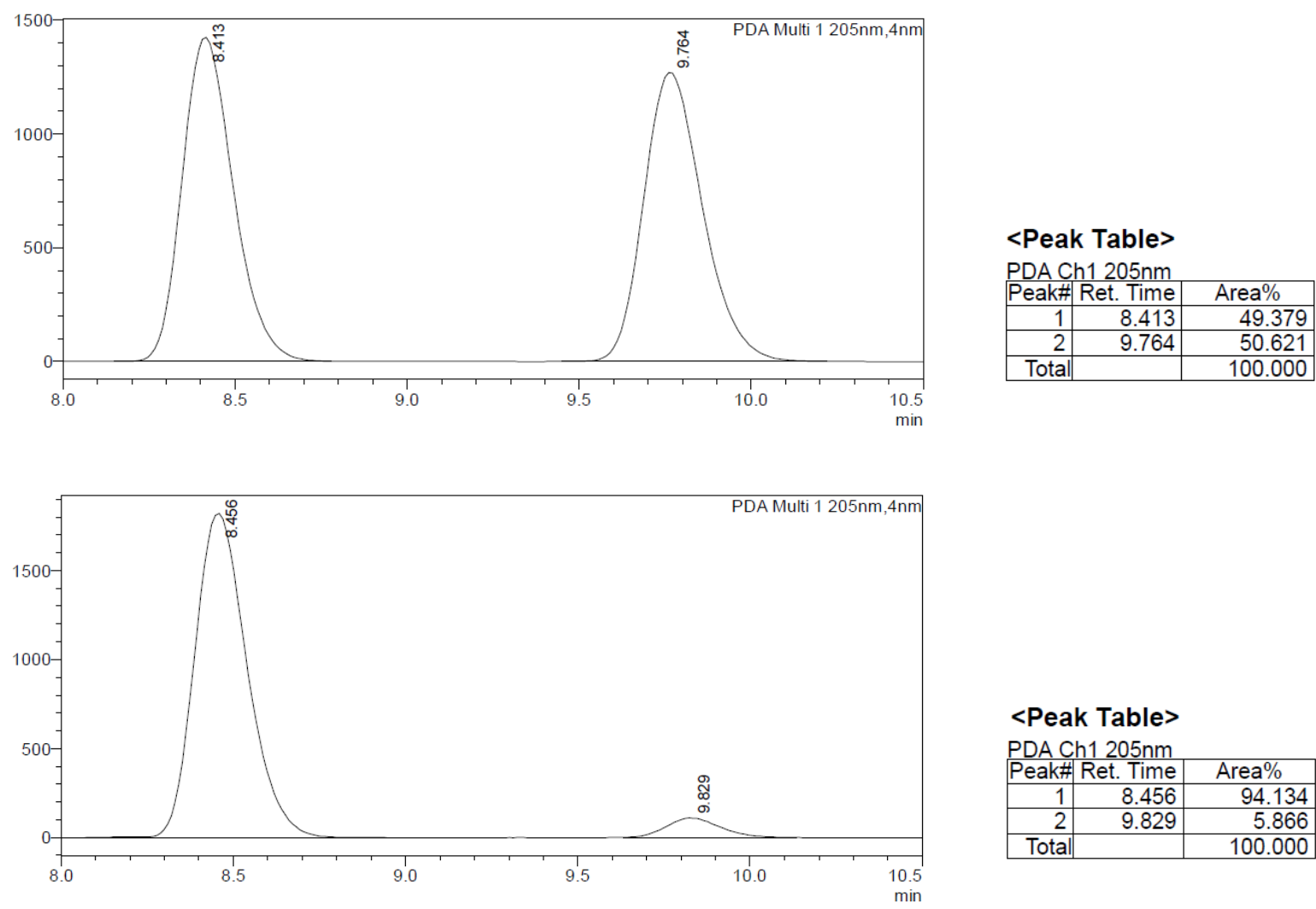


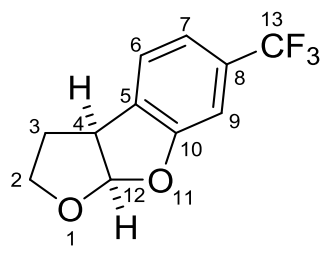

\section{(3aS,8aR)-6-(Trifluoromethyl)-2,3,3a,8a-tetrahydrofuro[2,3-b]benzofuran (3j)}

Prepared according to GP2 using 2,3-dihydrofuran (1a) (76 $\mu \mathrm{L}, 1 \mathrm{mmol}$ ) and 2-bromo 5(trifluoromethyl)phenol (2j) $(48.2 \mathrm{mg}, 0.2 \mathrm{mmol})$. Isolated by column chromatography (Pentane: $\mathrm{Et}_{2} \mathrm{O}$ 30:1) as a yellow oil (60\% yield, 88\% ee) with $\mathrm{R}_{\mathrm{f}}=0.30 ;{ }^{1} \mathbf{H}-\mathrm{NMR}\left(\mathbf{C}_{6} \mathbf{D}_{6}, 400 \mathrm{MHz}\right): \delta(\mathrm{ppm})=1.23(\mathrm{~m}$, $1 \mathrm{H}, \mathrm{H}-3), 1.50\left(\mathrm{~m}, 1 \mathrm{H}, \mathrm{H}-3^{\prime}\right), 3.03(\mathrm{~m}, 1 \mathrm{H}, \mathrm{H}-4), 3.16\left(\mathrm{ddd},{ }^{2} \mathrm{~J}_{\mathrm{H}-\mathrm{H}}=12.0 \mathrm{~Hz},{ }^{3} \mathrm{~J}_{\mathrm{H}-\mathrm{H}}=8.8 \mathrm{~Hz},{ }^{3} \mathrm{~J}_{\mathrm{H}-\mathrm{H}}=5.0 \mathrm{~Hz}\right.$, $1 \mathrm{H}, \mathrm{H}-2), 3.55\left(\mathrm{t},{ }^{3} \mathrm{~J}_{\mathrm{H}-\mathrm{H}}=8.2 \mathrm{~Hz}, 1 \mathrm{H}, \mathrm{H}-2^{\prime}\right), 5.96\left(\mathrm{~d}^{3} \mathrm{~J}_{\mathrm{H}-\mathrm{H}}=5.7 \mathrm{~Hz}, 1 \mathrm{H}, \mathrm{H}-7\right), 6.53\left(\mathrm{~d},{ }^{3} \mathrm{~J}_{\mathrm{H}-\mathrm{H}}=7.7 \mathrm{~Hz}, 1 \mathrm{H}, \mathrm{H}-\right.$ 6), $6.96\left(\mathrm{~d},{ }^{3} \mathrm{~J}_{\mathrm{H}-\mathrm{H}}=7.4 \mathrm{~Hz}, 1 \mathrm{H}, \mathrm{H}-7\right), 7.02(\mathrm{~s}, 1 \mathrm{H}, \mathrm{H}-9) ;{ }^{13} \mathrm{C}\left\{{ }^{1} \mathrm{H}\right\}-\mathrm{NMR}\left(\mathrm{C}_{6} \mathbf{D}_{6}, 100 \mathrm{MHz}\right): \delta(\mathrm{ppm})=33.3$ (C3), 46.2 (C-4), 66.9 (C-2), 106.2 (q, ${ }^{3} \mathrm{~J}_{\mathrm{C}-\mathrm{F}}=3.8 \mathrm{~Hz}, \mathrm{C}-9$ ), 112.0 (C-12), 118.1 (q, ${ }^{3} \mathrm{~J}_{\mathrm{C}-\mathrm{F}}=3.8 \mathrm{~Hz}, \mathrm{C}-7$ ), 124.5 (q, ${ }^{1} J_{C-F}=270.5 \mathrm{~Hz}, \mathrm{C}-13$ ), 125.1 (C-6), 130.0 (C-5), 131.3 (q, ${ }^{2} J_{C-F}=15.6 \mathrm{~Hz}, \mathrm{C}-8$ ), 160.3 (C-10); ${ }^{19} \mathrm{~F}-$ NMR ( $\left.\mathbf{C}_{6} \mathbf{D}_{6}, 282 \mathrm{MHz}\right): \delta(\mathrm{ppm})=-62.0(\mathrm{~s}, 3 \mathrm{~F})$; GC-MS (EI) $\mathrm{C}_{11} \mathrm{H}_{9} \mathrm{~F}_{3} \mathrm{O}_{2}, 230.0\left(100, \mathrm{M}^{+}\right), 215.0\left(58, \mathrm{M}^{+}\right.$ - 15), 213.0 (17, $\left.\mathrm{M}^{+}-17\right), 211.1\left(21, \mathrm{M}^{+}-19\right), 199.0$ (29, $\left.\mathrm{M}^{+}-31\right), 159.0\left(26, \mathrm{M}^{+}-71\right), 151.0\left(20, \mathrm{M}^{+}\right.$ - 79), $133.0\left(40, \mathrm{M}^{+}\right.$- 97), $115.1\left(17, \mathrm{M}^{+}-115\right)$; IR spectrum (neat) $\left(\mathrm{cm}^{-1}\right)=1436,1355,1314,1272$, $1239,1160,1114,1052,935,866,819,753,671 ;[\alpha]^{23}=-96.7\left(c 0.91, \mathrm{CH}_{2} \mathrm{Cl}_{2}\right)$; GC: Hydrodex $\beta-$ TBDMS, $30 \mathrm{~m}, 60^{\circ} \mathrm{C}-1{ }^{\circ} \mathrm{C} / \mathrm{min}-170{ }^{\circ} \mathrm{C}, 45 \mathrm{~cm} / \mathrm{s}, \mathrm{H}_{2}, t_{\mathrm{R} 1}=76.4$ and $t_{\mathrm{R} 2}=79.0 \mathrm{~min}$. 
<smiles>COC1Oc2cc(C(F)(F)F)ccc2[C@H]2CCOC12</smiles>

(3aS,8aR)-6-(Trifluoromethyl)-2,3,3a,8a-tetrahydrofuro[2,3-b]benzofuran (3j)
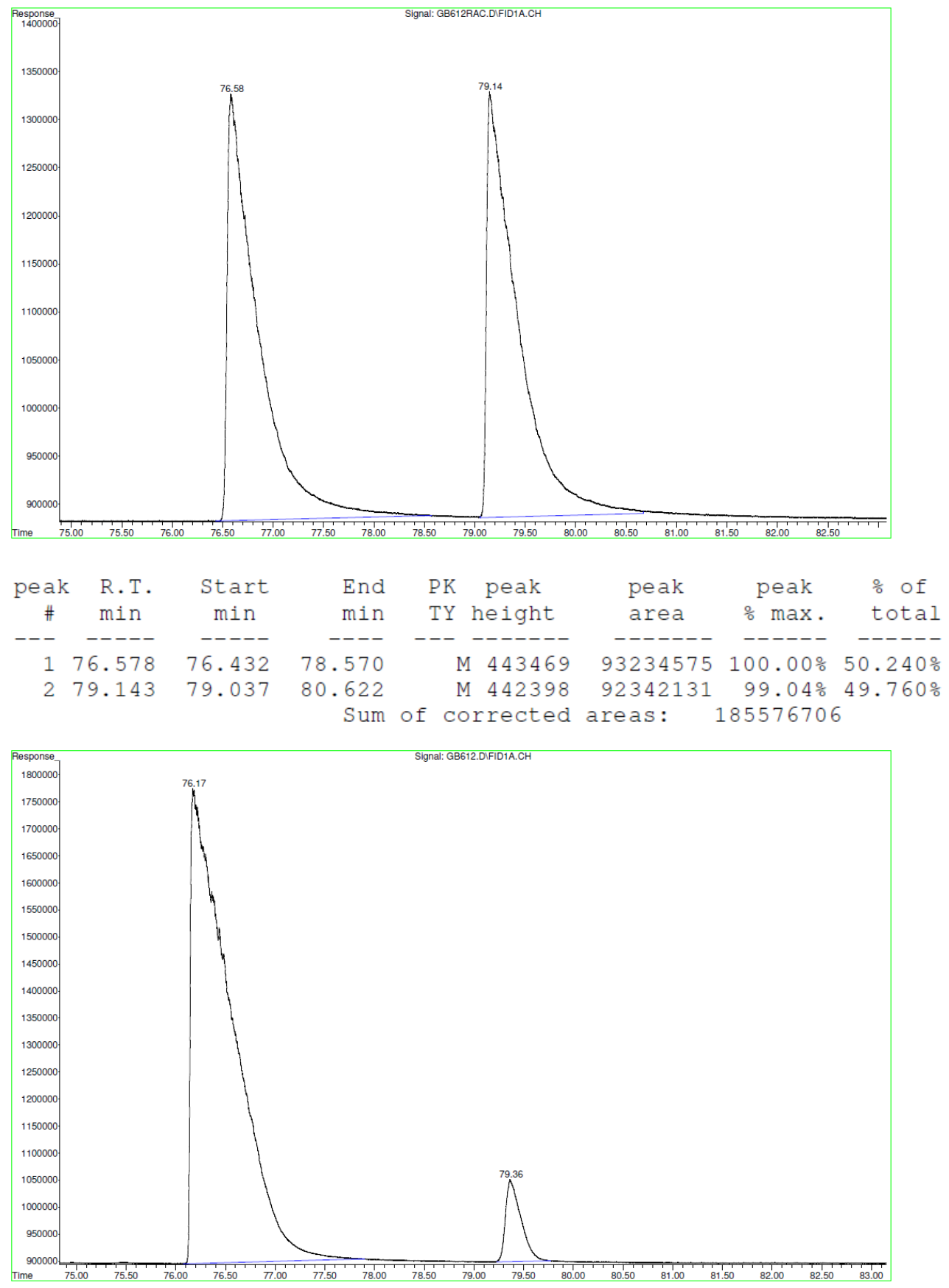

\begin{tabular}{|c|c|c|c|c|c|c|c|}
\hline $\begin{array}{c}\text { peak } \\
\#\end{array}$ & $\begin{array}{l}\text { R.T. } \\
\text { min }\end{array}$ & $\begin{array}{l}\text { Start } \\
\text { min }\end{array}$ & $\begin{array}{l}\text { End } \\
\text { min }\end{array}$ & $\begin{array}{l}\text { PK peak } \\
\text { TY height }\end{array}$ & $\begin{array}{l}\text { peak } \\
\text { area }\end{array}$ & $\begin{array}{l}\text { peak } \\
\% \max .\end{array}$ & $\begin{array}{l}\therefore \text { of } \\
\text { total }\end{array}$ \\
\hline-- & ---- & ----- & ---- & $---\quad-------$ & ------- & ------ & ------ \\
\hline 1 & 76.171 & 76.078 & 77.89 & M 878575 & 247921081 & $100.00 \%$ & $93.872 \%$ \\
\hline 2 & 79.362 & 79.232 & 79.768 & M 152153 & 16184928 & $6.53 \%$ & $6.128 \%$ \\
\hline
\end{tabular}


<smiles>Fc1cc2c(cc1F)C1CCOC1OCCO2</smiles>

\section{$(3 a S, 8 a R)-5,6-D i f l u o r o-2,3,3 a, 8 a-t e t r a h y d r o f u r o[2,3-b]$ benzofuran (3k)}

Prepared according to GP2 using 2,3-dihydrofuran (1a) (76 $\mu \mathrm{L}, 1 \mathrm{mmol}$ ) and 2-bromo-4,5difluorophenol (2k) (23 $\mu \mathrm{L}, 0.2 \mathrm{mmol}$ ). Isolated by column chromatography (Pentane: $\mathrm{Et}_{2} \mathrm{O} 40: 1$ ) as a white solid (71\% yield, 85\% ee) with $\mathrm{R}_{\mathrm{f}}=0.11 ;{ }^{1} \mathrm{H}-\mathrm{NMR}\left(\mathbf{C}_{6} \mathrm{D}_{6}, 400 \mathrm{MHz}\right): \delta(\mathrm{ppm})=1.16\left(\mathrm{~m}, 1 \mathrm{H}, \mathrm{H}-3^{\prime}\right)$, $1.44(\mathrm{~m}, 1 \mathrm{H}, \mathrm{H}-3), 2.96(\mathrm{~m}, 1 \mathrm{H}, \mathrm{H}-4), 3.21$ (ddd, ${ }^{2} J_{\mathrm{H}-\mathrm{H}}=12.0 \mathrm{~Hz},{ }^{3} J_{\mathrm{H}-\mathrm{H}}=8.7 \mathrm{~Hz},{ }^{3} J_{\mathrm{H}-\mathrm{H}}=4.9 \mathrm{~Hz}, 1 \mathrm{H}, \mathrm{H}-2$ ), $3.57\left(\mathrm{t},{ }^{3} \mathrm{~J}_{\mathrm{H}-\mathrm{H}}=8.2 \mathrm{~Hz}, 1 \mathrm{H}, \mathrm{H}-2^{\prime}\right), 5.92\left(\mathrm{~d},{ }^{3} J_{\mathrm{H}-\mathrm{H}}=5.6 \mathrm{~Hz}, 1 \mathrm{H}, \mathrm{H}-12\right), 6.32(\mathrm{~m}, 1 \mathrm{H}, \mathrm{H}-6), 6.37\left(\mathrm{dd},{ }^{3} J_{\mathrm{H}-\mathrm{F}}=\right.$ $\left.10.5 \mathrm{~Hz},{ }^{4} \mathrm{H}_{\mathrm{H}-\mathrm{F}}=6.5 \mathrm{~Hz}, 1 \mathrm{H}, \mathrm{H}-9\right) ;{ }^{13} \mathrm{C}\left\{{ }^{1} \mathrm{H}\right\}-\mathrm{NMR}\left(\mathrm{C}_{6} \mathrm{D}_{6}, 100 \mathrm{MHz}\right): \delta(\mathrm{ppm})=33.3$ (C-3), 46.3 (C-4), 66.9 (C-2), $98.9\left(\mathrm{~d},{ }^{3} \mathrm{~J}_{\mathrm{C}-\mathrm{F}}=21.8 \mathrm{~Hz}, \mathrm{C}-9\right), 112.4(\mathrm{C}-12), 112.9\left(\mathrm{dd},{ }^{3} \mathrm{~J}_{\mathrm{C}-\mathrm{F}}=19.7 \mathrm{~Hz},{ }^{4} \mathrm{~J}_{\mathrm{C}-\mathrm{F}}=1.6 \mathrm{~Hz}, \mathrm{C}-6\right), 123.3$ $\left(\mathrm{dd},{ }^{3} \mathrm{~J}_{\mathrm{C}-\mathrm{F}}=6.2 \mathrm{~Hz},{ }^{4} \mathrm{~J}_{\mathrm{C}-\mathrm{F}}=3.3 \mathrm{~Hz}, \mathrm{C}-5\right), 145.8$ (dd, ${ }^{1} J_{\mathrm{C}-\mathrm{F}}=239.1 \mathrm{~Hz},{ }^{3} \mathrm{~J}_{\mathrm{C}-\mathrm{F}}=13.6 \mathrm{~Hz}, \mathrm{C}-7$ or C-8), 150.8 (dd, ${ }^{1} J_{C-F}=246.3 \mathrm{~Hz},{ }^{3} J_{C-F}=14.3 \mathrm{~Hz}, \mathrm{C}-7$ or C-8), 155.7 (dd, $\left.{ }^{3} J_{C-F}=10.7 \mathrm{~Hz},{ }^{4} J_{C-F}=1.9 \mathrm{~Hz}, \mathrm{C}-10\right) ;{ }^{19} \mathrm{~F}-\mathrm{NMR}$ $\left(\mathbf{C}_{6} \mathbf{D}_{6}, 282 \mathrm{MHz}\right): \delta(\mathrm{ppm})=-147.0\left(\mathrm{~d},{ }^{3} \mathrm{JF}_{\mathrm{F} F}=20.7 \mathrm{~Hz}, 1 \mathrm{~F}\right),-135.9\left(\mathrm{~d},{ }^{3} \mathrm{~J}_{\mathrm{F}-\mathrm{F}}=20.7 \mathrm{~Hz}, 1 \mathrm{~F}\right)$; GC-MS (EI): $\left(\mathrm{C}_{10} \mathrm{H}_{8} \mathrm{~F}_{2} \mathrm{O}_{2}\right), 198.0\left(100, \mathrm{M}^{+}\right), 183.0\left(44, \mathrm{M}^{+}-15\right), 167.0$ (25, $\left.\mathrm{M}^{+}-31\right), 114.0$ (31, $\left.\mathrm{M}^{+}-84\right), 101.1$ (26, $\mathrm{M}^{+}$- 97); IR spectrum (neat) $\left(\mathrm{cm}^{-1}\right)=2889,1630,1497,1443,1256,1198,1156,1074,949,927,862$, 738, 651; $[\alpha]^{23}{ }_{\mathrm{D}}=-90.4$ (c 1.10, $\mathrm{CH}_{2} \mathrm{Cl}_{2}$ ); HPLC: OJ-H, $214 \mathrm{~nm}$, Hexane: $\mathrm{PrOH}, 98: 2,1 \mathrm{~mL} / \mathrm{min}, 30^{\circ} \mathrm{C}$, $t_{\mathrm{R} 1}=13.5$ and $t_{\mathrm{R} 2}=14.5 \mathrm{~min}$.
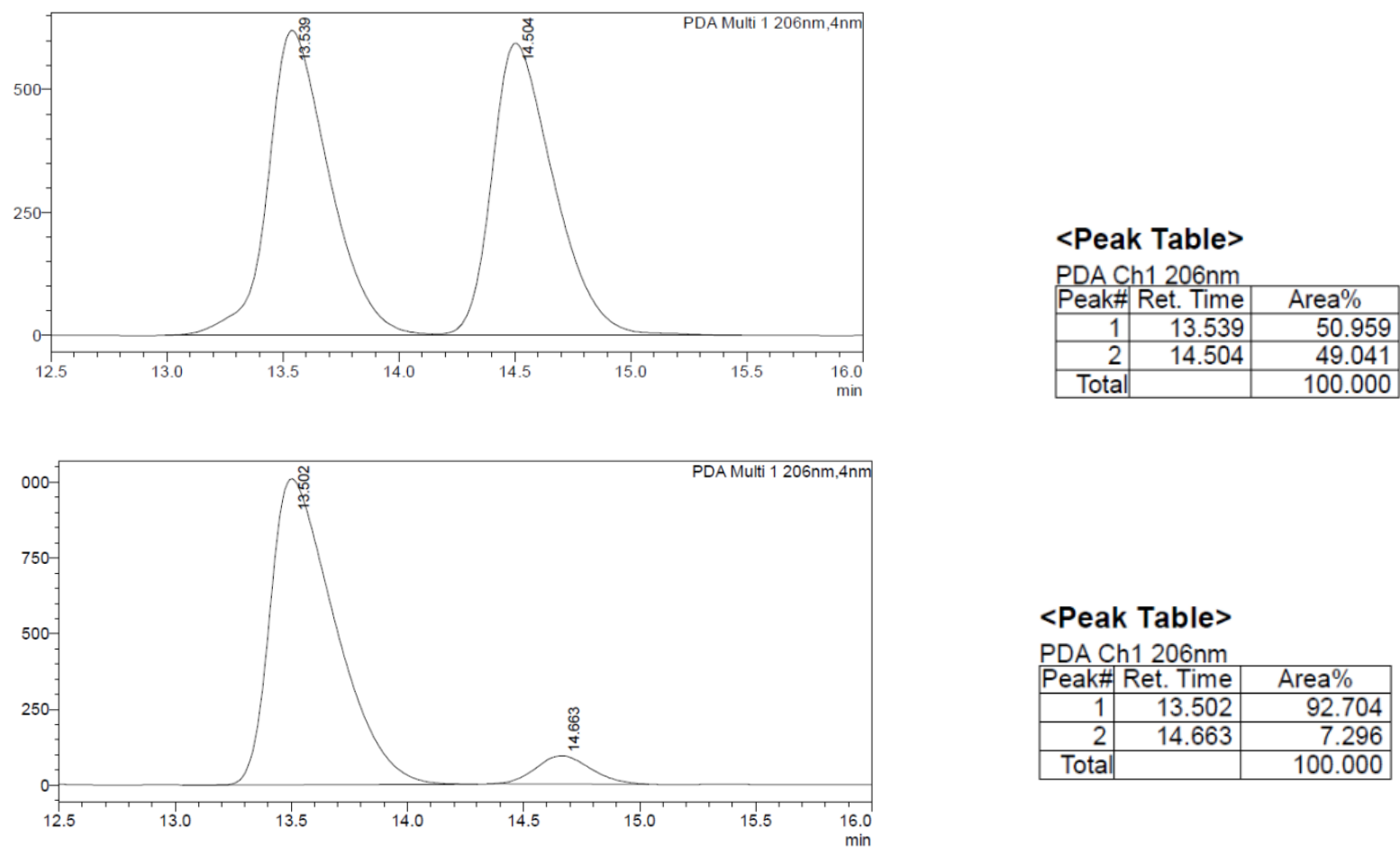

<Peak Table> PDA Ch1 206nm \begin{tabular}{|l|l|}
\hline Peak\# Ret. Time & Area $\%$ \\
\hline
\end{tabular}

\begin{tabular}{|c|c|c|}
\hline & & \\
\hline 1 & 13.502 & 92.704 \\
\hline 2 & 14.663 & 96 \\
\hline Total & & 100 \\
\hline
\end{tabular}


<smiles>CO[C@]12OCC[C@H]1c1ccccc1O2</smiles>

(3aS,8aR)-8a-Methyl-2,3,3a,8a-tetrahydrofuro[2,3-b]benzofuran (3I)

Prepared according to GP2 using 5-Me-2,3-dihydrofuran (1b) (90 $\mu \mathrm{L}, 1 \mathrm{mmol})$, 2-bromophenol (2a) (23 $\mu \mathrm{L}, 0.2 \mathrm{mmol}), 10 \mathrm{~mol} \%$ of $\mathrm{Pd}(\mathrm{OAc})_{2}$ and $10 \mathrm{~mol} \%$ of (S)-DTBM-MeO-BIPHEP. Isolated by column chromatography (Pentane:Et ${ }_{2} \mathrm{O} 80: 1$ ) as a pale yellow oil (64\% yield, $91 \%$ ee) with $\mathrm{R}_{\mathrm{f}}=0.10 ;{ }^{1} \mathrm{H}-\mathrm{NMR}$ $\left(\mathbf{C}_{6} \mathbf{D}_{6}, 400 \mathrm{MHz}\right): \delta(\mathrm{ppm})=1.50(\mathrm{~m}, 1 \mathrm{H}, \mathrm{H}-3), 1.55(\mathrm{~s}, 3 \mathrm{H}, \mathrm{H}-13), 1.76\left(\mathrm{~m}, 1 \mathrm{H}, \mathrm{H}-3^{\prime}\right), 3.08\left(\mathrm{t}^{3} \mathrm{~J}_{\mathrm{H}-\mathrm{H}}=8.8\right.$ $\mathrm{Hz}, 1 \mathrm{H}, \mathrm{H}-2^{\prime}$ ), 3.46 (ddd, $\left.{ }^{2} J_{\mathrm{H}-\mathrm{H}}=12.0 \mathrm{~Hz},{ }^{3} \mathrm{~J}_{\mathrm{H}-\mathrm{H}}=8.7 \mathrm{~Hz},{ }^{3} J_{\mathrm{H}-\mathrm{H}}=4.9 \mathrm{~Hz}, 1 \mathrm{H}, \mathrm{H}-2\right), 3.65\left(\mathrm{t},{ }^{3} J_{\mathrm{H}-\mathrm{H}}=8.2 \mathrm{~Hz}\right.$, $\left.1 \mathrm{H}, \mathrm{H}-2^{\prime}\right), 6.80(\mathrm{~m}, 2 \mathrm{H}, \mathrm{H}-8$ or $\mathrm{H}-9), 6.87(\mathrm{~m}, 1 \mathrm{H}, \mathrm{H}-6), 6.98(\mathrm{~m}, 1 \mathrm{H}, \mathrm{H}-8) ;{ }^{13} \mathrm{C}\left\{{ }^{1} \mathrm{H}\right\}-\mathrm{NMR}\left(\mathrm{C}_{6} \mathrm{D}_{6}, 100\right.$ MHz): $\delta(p p m)=24.5$ (C-13), 34.5 (C-3), 50.8 (C-4), 67.5 (C-2), 109.3 (C-7 or C-9), 119.9 (C-5), 120.8 (C-7 or C-9), 124.9 (C-6), 129.0 (C-8), 129.1 (C-12), 160.0 (C-10); GC-MS (EI): $\mathrm{C}_{11} \mathrm{H}_{11} \mathrm{O}_{2}, 176.1$ (100, $\left.M^{+}\right), 133.1\left(66, M^{+}-43\right), 105.1\left(45, M^{+}-71\right), 77.1\left(34, M^{+}-99\right)$; IR spectrum (neat) $\left(\mathrm{cm}^{-1}\right)=2984$, $1596,1479,1461,1383,1313,1274,1264,1121,1109,1074,1006,890,871,810,735 ;[\alpha]^{23}=-$ 102.4 (c 0.82, $\mathrm{CH}_{2} \mathrm{Cl}_{2}$ ); HPLC: OJ-H, $205 \mathrm{~nm}$, Hexane: $\mathrm{PPrOH}, 98: 2,0.5 \mathrm{~mL} / \mathrm{min}, 30{ }^{\circ} \mathrm{C}, t_{\mathrm{R} 1}=14.0$ and $t_{\mathrm{R} 2}$ $=16.5 \mathrm{~min}$.
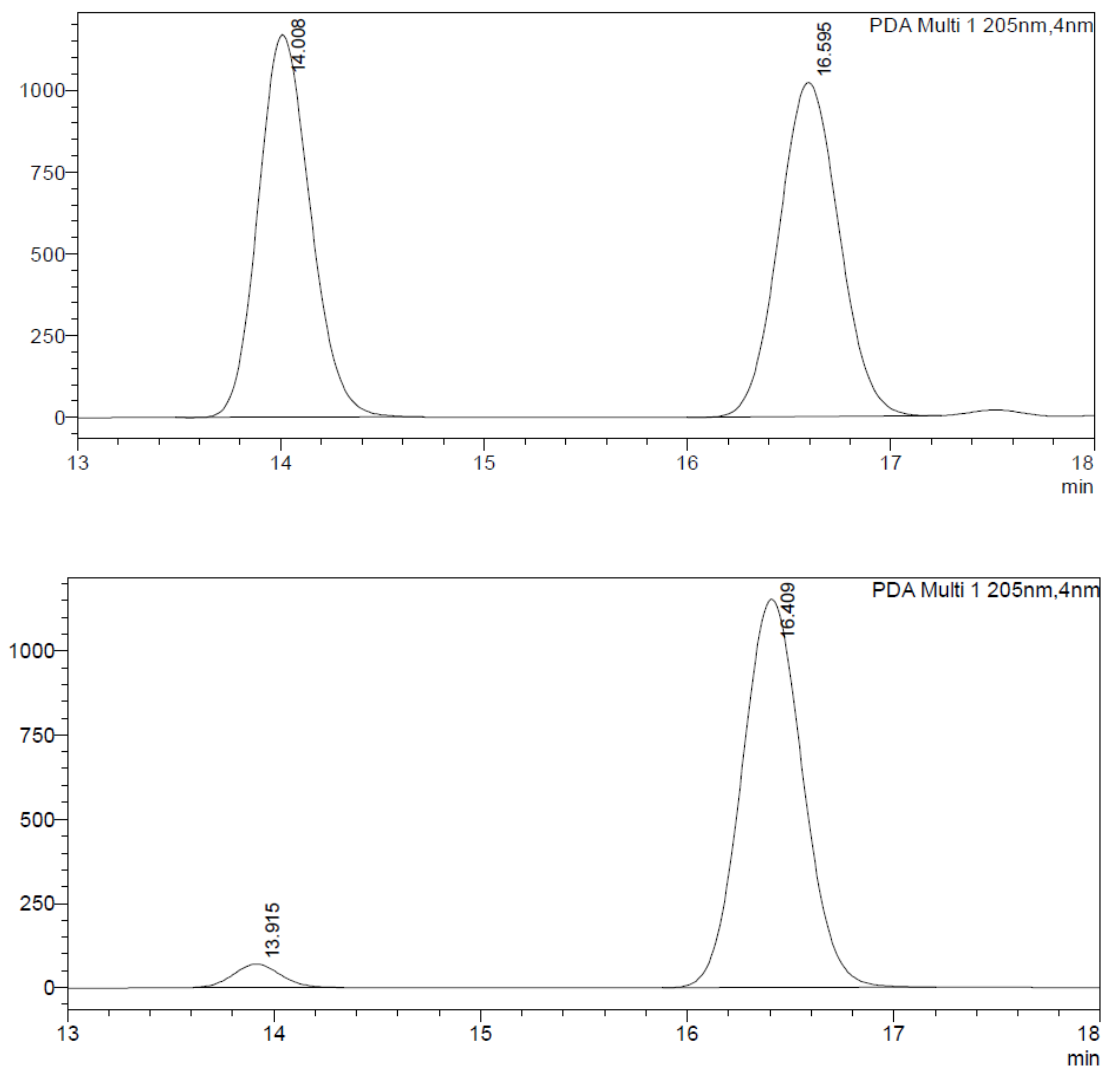

<Peak Table> PDA Ch1 205nm \begin{tabular}{|r|r|r|}
\hline Peak\# & Ret. Time & Area $\%$ \\
\hline 1 & 14.008 & 49.778 \\
\hline 2 & 16.595 & 50.222 \\
\hline Total & & 100.000 \\
\hline
\end{tabular}

<Peak Table> PDA Ch1 205nm \begin{tabular}{|r|r|r|}
\hline Peak\# & Ret. Time & \multicolumn{1}{|c|}{ Area $\%$} \\
\hline 1 & 13.915 & 4.436 \\
\hline 2 & 16.409 & 95.564 \\
\hline Total & & 100.000 \\
\hline
\end{tabular} 
<smiles>COC1(O)OCCC1c1ccc([N+](=O)[O-])cc1</smiles>

(3aS,8aR)-6,8a-Dimethyl-2,3,3a,8a-tetrahydrofuro[2,3-b]benzofuran (3m)

Prepared according to GP2 using 5-Me-2,3-dihydrofuran (1b) (90 $\mu \mathrm{L}, 1 \mathrm{mmol}), 2$-bromo-5methylphenol $(\mathbf{2 g})(37.4 \mathrm{mg}, 0.2 \mathrm{mmol}), 10 \mathrm{~mol} \%$ of $\mathrm{Pd}(\mathrm{OAc})_{2}$ and $10 \mathrm{~mol} \%$ of $(S)$-DTBM-MeOBIPHEP. Isolated by column chromatography (Pentane:Et $\mathrm{C}_{2} \mathrm{O} 90: 1$ ) as a pale yellow oil (65\% yield, $90 \%$ ee) with $\mathrm{R}_{\mathrm{f}}=0.10 ;{ }^{1} \mathrm{H}-\mathrm{NMR}\left(\mathbf{C}_{6} \mathrm{D}_{6}, 400 \mathrm{MHz}\right): \delta(\mathrm{ppm})=1.49(\mathrm{~m}, 1 \mathrm{H}, \mathrm{H}-3), 1.58(\mathrm{~s}, 3 \mathrm{H}, \mathrm{H}-13), 1.77(\mathrm{~m}$, $\left.1 \mathrm{H}, \mathrm{H}-3^{\prime}\right), 2.09(\mathrm{~s}, 3 \mathrm{H}, \mathrm{H}-14), 3.11(\mathrm{~m}, 1 \mathrm{H}, \mathrm{H}-5), 3.52\left(\mathrm{ddd},{ }^{2} \mathrm{~J}_{\mathrm{H}-\mathrm{H}}=12.0 \mathrm{~Hz},{ }^{3} \mathrm{~J}_{\mathrm{H}-\mathrm{H}}=8.7 \mathrm{~Hz},{ }^{3} \mathrm{~J}_{\mathrm{H}-\mathrm{H}}=4.9 \mathrm{~Hz}\right.$, $1 \mathrm{H}, \mathrm{H}-2), 3.68\left(\mathrm{t},{ }^{3} \mathrm{~J}_{\mathrm{H}-\mathrm{H}}=8.1 \mathrm{~Hz}, 1 \mathrm{H}, \mathrm{H}-2^{\prime}\right), 6.64(\mathrm{~m}, 1 \mathrm{H}, \mathrm{H}-7), 6.68(\mathrm{~s}, 1 \mathrm{H}, \mathrm{H}-9), 6.81\left(\mathrm{~d},{ }^{3} \mathrm{H}_{\mathrm{H}-\mathrm{H}}=7.4 \mathrm{~Hz}\right.$, $1 \mathrm{H}, \mathrm{H}-6) ;{ }^{13} \mathrm{C}\left\{{ }^{1} \mathrm{H}\right\}-\mathrm{NMR}\left(\mathrm{C}_{6} \mathrm{D}_{6}, 100 \mathrm{MHz}\right): \delta(\mathrm{ppm})=21.5$ (C-14), 24.6 (C-13), 34.6 (C-3), 50.6 (C-4), 67.5 (C-2), 110.1 (C-9), 120.2 (C-5), 121.6 (C-7), 124.5 (C-6), 126.2 (C-12), 138.9 (C-8), 160.3 (C-10); GC-MS (EI): $\mathrm{C}_{12} \mathrm{H}_{14} \mathrm{O}_{2}, 190.1\left(100, \mathrm{M}^{+}\right), 175.1\left(24, \mathrm{M}^{+}-15\right), 147.1$ (63, $\mathrm{M}^{+}-47$ ), 91.1 (28, $\left.\mathrm{M}^{+}-99\right), 119.1$ (26, $\left.M^{+}-71\right), 115.1\left(21, M^{+}-75\right)$; IR spectrum (neat) $\left(\mathrm{cm}^{-1}\right)=1620,1593,1498,1443,1382,1312,1278$, $1171,1114,1076,1007,929,946,871,801,752,624,587 ;[\alpha]^{23}=-88.6\left(c 0.87, \mathrm{CH}_{2} \mathrm{Cl}_{2}\right)$; HPLC: OD$\mathrm{H}, 206 \mathrm{~nm}$, Hexane: $\mathrm{PPOH}, 98: 2,0.5 \mathrm{~mL} / \mathrm{min}, 30^{\circ} \mathrm{C}, t_{\mathrm{R} 1}=10.2$ and $t_{\mathrm{R} 2}=10.9 \mathrm{~min}$.
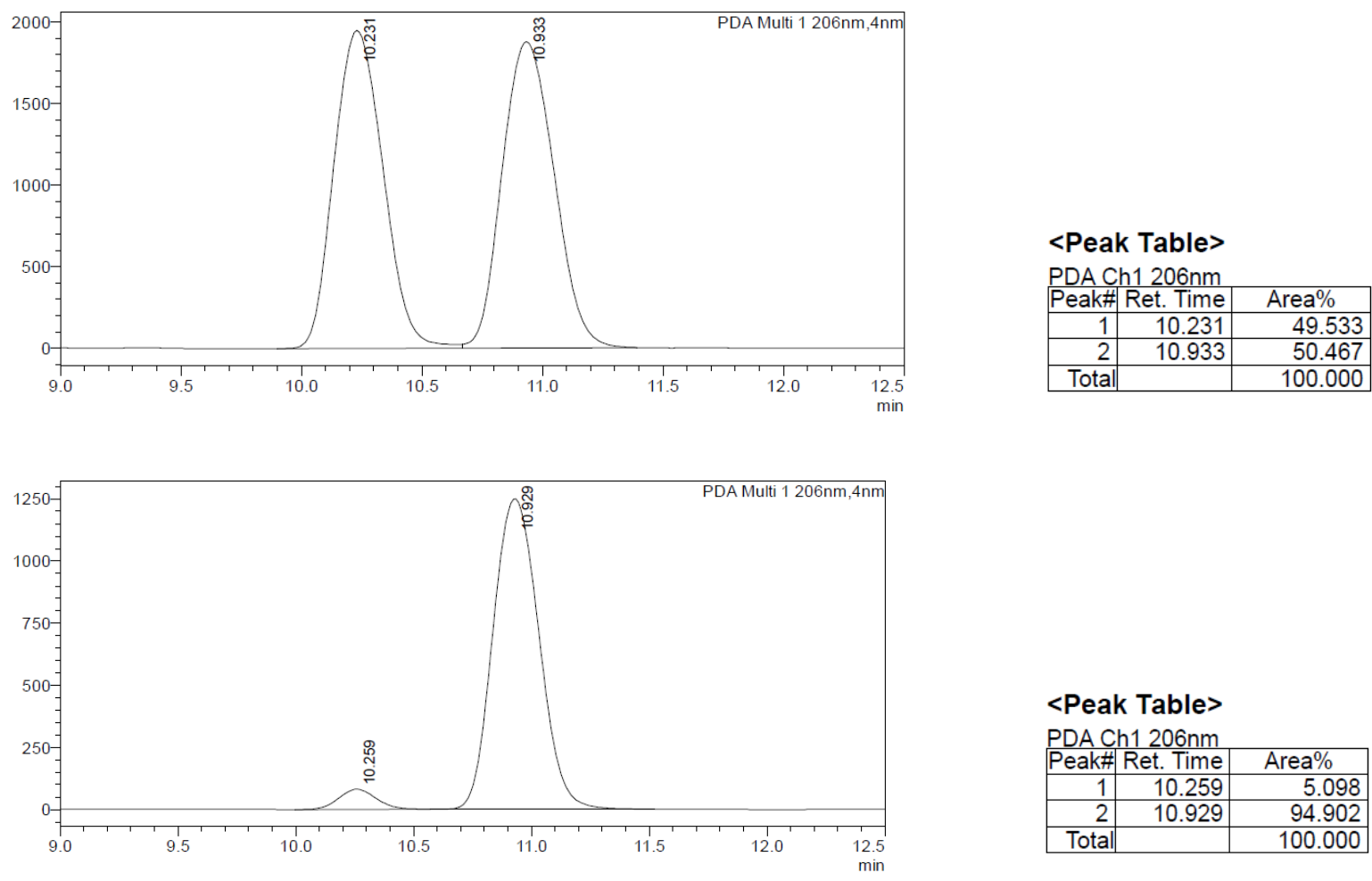
<smiles>Cc1ccc(C2CC3c4ccccc4OC3(O)O2)cc1</smiles>

\section{$(2 R, 3 a S, 8 a R)-2-(p-T o l y l)-2,3,3 a, 8 a-t e t r a h y d r o f u r o[2,3-b]$ benzofuran (3n)}

Prepared according to GP2 using rac-2-(p-tolyl)-2,3-dihydrofuran (1c) $(160.2 \mathrm{mg}, 1 \mathrm{mmol}$ ) and 2bromophenol (2a) (23 $\mathrm{LL}, 0.2 \mathrm{mmol}$ ). Isolated by column chromatography (Pentane: $\left.\mathrm{Et}_{2} \mathrm{O} 30: 1\right)$ as a colorless oil (33 mg, 65\% yield, 49:1 dr, 91\% ee) with $\mathrm{R}_{\mathrm{f}}=0.4$ (Pentane:Et ${ }_{2} \mathrm{O}$ 9:1); ${ }^{\mathbf{1}} \mathbf{H}-\mathbf{N M R}\left(\mathbf{C}_{6} \mathbf{D}_{6}, 400\right.$ MHz): 1.79 (ddd, ${ }^{2} J_{\mathrm{H}-\mathrm{H}}=12.2 \mathrm{~Hz},{ }^{3} \mathrm{~J}_{\mathrm{H}-\mathrm{H}}=11.2 \mathrm{~Hz},{ }^{3} J_{\mathrm{H}-\mathrm{H}}=8.4 \mathrm{~Hz}, 1 \mathrm{H}, \mathrm{H}-3$ ), 2.00 (dd, ${ }^{2} J_{\mathrm{H}-\mathrm{H}}=12.3 \mathrm{~Hz},{ }^{3} J_{\mathrm{H}-\mathrm{H}}=$ $\left.4.7 \mathrm{~Hz}, 1 \mathrm{H}, \mathrm{H}-3^{\prime}\right), 2.09(\mathrm{~s}, 3 \mathrm{H}, \mathrm{H}-17), 3.44\left(\mathrm{dd},{ }^{3} \mathrm{~J}_{\mathrm{H}-\mathrm{H}}=8.6 \mathrm{~Hz},{ }^{3} \mathrm{~J}_{\mathrm{H}-\mathrm{H}}=5.6 \mathrm{~Hz}, 1 \mathrm{H}, \mathrm{H}-4\right), 4.82\left(\mathrm{dd},{ }^{3} \mathrm{~J}_{\mathrm{H}-\mathrm{H}}=\right.$ $\left.11.2 \mathrm{~Hz},{ }^{3} J_{\mathrm{H}-\mathrm{H}}=4.6 \mathrm{~Hz}, 1 \mathrm{H}, \mathrm{H}-2\right), 6.24\left(\mathrm{~d},{ }^{3} J_{\mathrm{H}-\mathrm{H}}=5.7 \mathrm{~Hz}, 1 \mathrm{H}, \mathrm{H}-12\right), 6.80\left(\mathrm{dt},{ }^{3} \mathrm{~J}_{\mathrm{H}-\mathrm{H}}=7.4 \mathrm{~Hz},{ }^{4} \mathrm{~J}_{\mathrm{H}-\mathrm{H}}=1.1 \mathrm{~Hz}\right.$, $1 \mathrm{H}, \mathrm{H}-7$ ), $6.88\left(\mathrm{~d},{ }^{3} \mathrm{~J}_{\mathrm{H}-\mathrm{H}}=7.4 \mathrm{~Hz}, 2 \mathrm{H}, \mathrm{H}-6+\mathrm{H}-9\right), 6.94\left(\mathrm{~d},{ }^{3} \mathrm{~J}_{\mathrm{H}-\mathrm{H}}=7.9 \mathrm{~Hz}, 2 \mathrm{H}, \mathrm{H}-15\right), 7.00\left(\mathrm{t},{ }^{3} \mathrm{~J}_{\mathrm{H}-\mathrm{H}}=7.8 \mathrm{~Hz}\right.$, $1 \mathrm{H}, \mathrm{H}-8), 7.05\left(\mathrm{~d},{ }^{3} \mathrm{~J}_{\mathrm{H}-\mathrm{H}}=8.1 \mathrm{~Hz}, 2 \mathrm{H}, \mathrm{H}-14\right) ;{ }^{13} \mathrm{C}\left\{{ }^{1} \mathrm{H}\right\}-\mathrm{NMR}\left(\mathrm{C}_{6} \mathrm{D}_{6}, 100 \mathrm{MHz}\right): 21.1$ (C-17), 42.6 (C-3), 47.6 (C-4), 80.2 (C-2), 109.6 (C-9), 111.0 (C-12), 121.3 (C-7), 124.9 (C-6), 126.4 (C-14), 128.4 (C-5), 129.1 (C-8), 129.2 (C-15), 137.4 (C-13), 137.6 (C-16), 160.5 (C-10); GC-MS (EI): $\left(\mathrm{C}_{17} \mathrm{H}_{16} \mathrm{O}_{2}\right.$ ), $252.1\left(42, \mathrm{M}^{+}\right)$, $223.1\left(100, M^{+}-29\right), 208.1\left(15, M^{+}-44\right), 189.0$ (10, $\left.M^{+}-63\right), 178.0\left(6, M^{+}-74\right), 165.0\left(7, M^{+}-87\right)$, $131.1\left(47, \mathrm{M}^{+}-121\right), 117.2$ (16, $\mathrm{M}^{+}$- 135), 105.1 (22, $\mathrm{M}^{+}$- 147), $91.1\left(22, \mathrm{M}^{+}-161\right), 77.1\left(18, \mathrm{M}^{+}-\right.$ 175), $63.0\left(11, \mathrm{M}^{+}\right.$- 189); IR spectrum (neat) $\left(\mathrm{cm}^{-1}\right)=2970,1597,1479,1461,1323,1247,1223$, 1072, 996, 941, 913, 811, 749; $[\alpha]^{23}{ }_{D}=-136.7$ (c 0.49, $\mathrm{CH}_{2} \mathrm{Cl}_{2}$ ); HPLC: OD-H, $204 \mathrm{~nm}$, Hexane:iPrOH, $98: 2,1 \mathrm{~mL} / \mathrm{min}, 30^{\circ} \mathrm{C}, t_{\mathrm{R} 1}=11.6$ and $t_{\mathrm{R} 2}=20.2 \mathrm{~min}$.
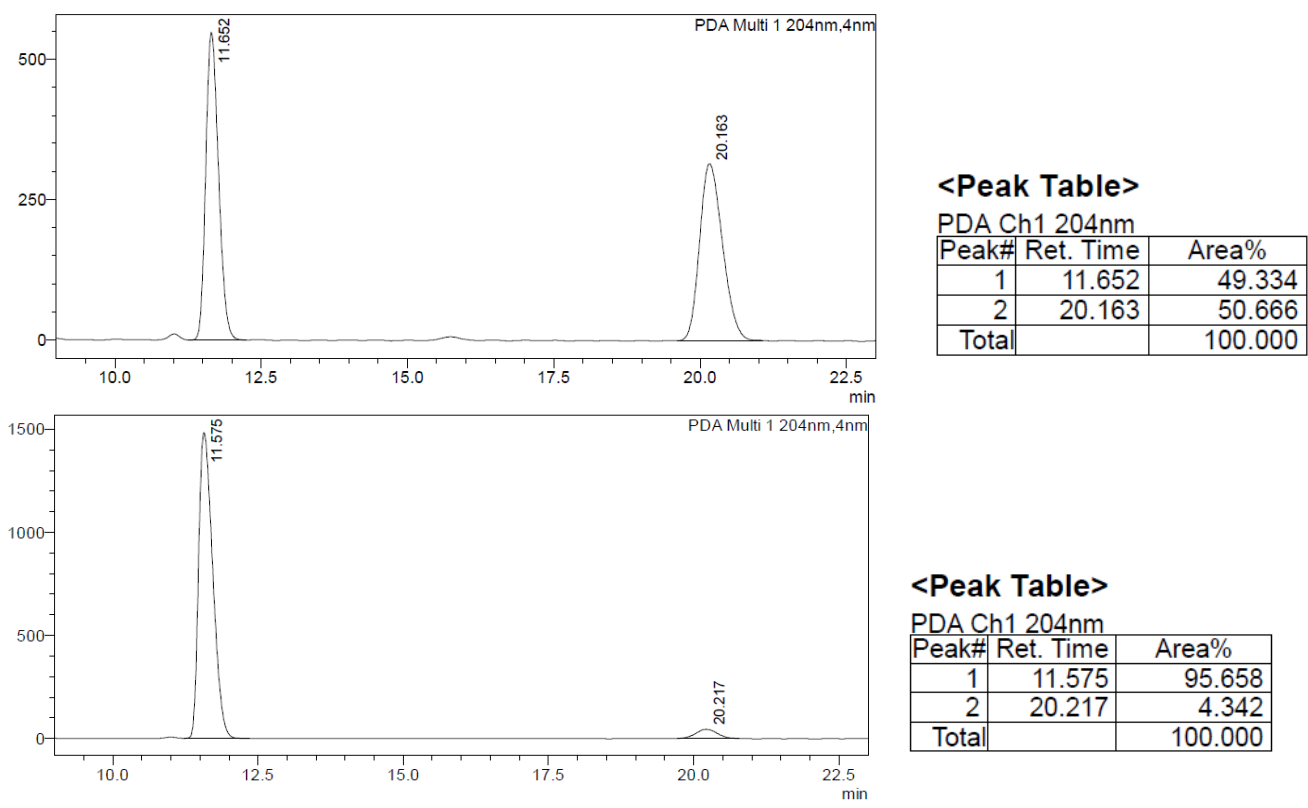


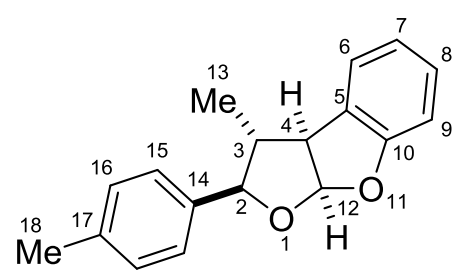

\section{(2R,3R,3aS,8aR)-3-Methyl-2-( $p$-tolyl)-2,3,3a,8a-tetrahydrofuro[2,3-b]benzofuran (3o)}

Prepared according to GP2 using trans-3-methyl-2-(p-tolyl)-2,3-dihydrofuran (1d) (174.2 mg, 1 $\mathrm{mmol}$ ) and 2-bromophenol (2a) $(23 \mu \mathrm{L}, 0.2 \mathrm{mmol})$. Isolated by column chromatography (Pentane:Et ${ }_{2} \mathrm{O} 30: 1$ ) as a white solid ( $28 \mathrm{mg}, 53 \%$ yield, 10:1 dr, 96\% ee) with $\mathrm{R}_{\mathrm{f}}=0.4$ (Pentane: $\mathrm{Et}_{2} \mathrm{O}$ 9:1); mp $=85^{\circ} \mathrm{C} ;{ }^{1} \mathrm{H}-\mathrm{NMR}\left(\mathrm{C}_{6} \mathrm{D}_{6}, 400 \mathrm{MHz}\right): 0.71\left(\mathrm{~d},{ }^{3} \mathrm{H}_{\mathrm{H}-\mathrm{H}}=6.9 \mathrm{~Hz}, 3 \mathrm{H}, \mathrm{H}-13_{\text {Maj. }}\right), 0.83\left(\mathrm{~d},{ }^{3} \mathrm{~J}_{\mathrm{H}-\mathrm{H}}=6.8 \mathrm{~Hz}\right.$,

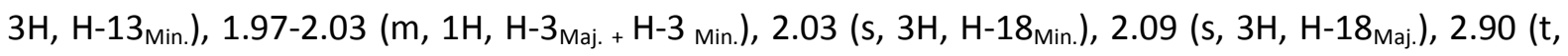
${ }^{3} J_{\mathrm{H}-\mathrm{H}}=6.7 \mathrm{~Hz}, 1 \mathrm{H}, \mathrm{H}-4_{\text {Min. }}$ ), $3.40\left(\mathrm{dd},{ }^{3} J_{\mathrm{H}-\mathrm{H}}=8.0 \mathrm{~Hz},{ }^{3} J_{\mathrm{H}-\mathrm{H}}=6.1 \mathrm{~Hz}, 1 \mathrm{H}, \mathrm{H}-4_{\text {Maj. }}\right.$ ), 4.29 (d, ${ }^{3} J_{\mathrm{H}-\mathrm{H}}=10.5 \mathrm{~Hz}$, $\left.1 \mathrm{H}, \mathrm{H}-2_{\text {Maj. }}\right), 4.37$ (d, $\left.{ }^{3} \mathrm{~J}_{\mathrm{H}-\mathrm{H}}=8.1 \mathrm{~Hz}, 1 \mathrm{H}, \mathrm{H}-2_{\text {Min. }}\right), 6.17\left(\mathrm{~d},{ }^{3} \mathrm{~J}_{\mathrm{H}-\mathrm{H}}=6.2 \mathrm{~Hz}, 1 \mathrm{H}, \mathrm{H}-12_{\text {Min. }}\right.$ ), $6.32\left(\mathrm{~d},{ }^{3} \mathrm{~J}_{\mathrm{H}-\mathrm{H}}=5.9\right.$ $\mathrm{Hz}, 1 \mathrm{H}, \mathrm{H}-12_{\text {Maj. }}$ ), $6.72\left(\mathrm{dt},{ }^{3} \mathrm{~J}_{\mathrm{H}-\mathrm{H}}=7.4 \mathrm{~Hz},{ }^{4} \mathrm{~J}_{\mathrm{H}-\mathrm{H}}=1.0 \mathrm{~Hz}, 1 \mathrm{H}, \mathrm{H}-7_{\text {Min. }}\right), 6.79\left(\mathrm{dt},{ }^{3} \mathrm{~J}_{\mathrm{H}-\mathrm{H}}=7.2 \mathrm{~Hz},{ }^{4} \mathrm{~J}_{\mathrm{H}-\mathrm{H}}=0.9\right.$ $\left.H z, 1 H, H-7_{\text {Maj. }}\right), 6.87-6.98\left(m, 4 H, H-6_{\text {Maj. }}+H-9_{\text {Maj. }}+H-16_{\text {Maj, }}\right.$ and $\left.4 H, H-6_{\text {Min. }}+H-9_{\text {Min. }}+H-16_{\text {Min. }}\right)$, 7.01$7.06\left(\mathrm{~m}, 3 \mathrm{H}, \mathrm{H}-15_{\text {Maj. }}+\mathrm{H}-8_{\text {Maj. }}\right.$ and $\left.1 \mathrm{H}, \mathrm{H}-8_{\text {Min. }}\right), 7.22\left(\mathrm{~d},{ }^{3} \mathrm{~J}_{\mathrm{H}-\mathrm{H}}=8.0 \mathrm{~Hz}, 2 \mathrm{H}, \mathrm{H}-15_{\text {Min. }}\right) ;{ }^{13} \mathrm{C}\left\{{ }^{1} \mathrm{H}\right\}-\mathrm{NMR}$

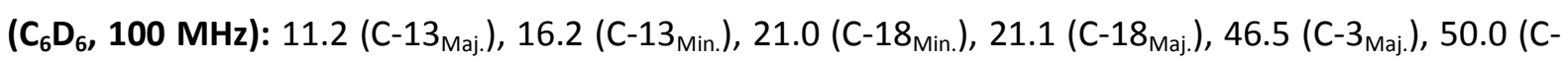

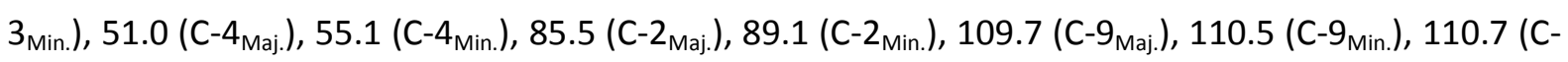

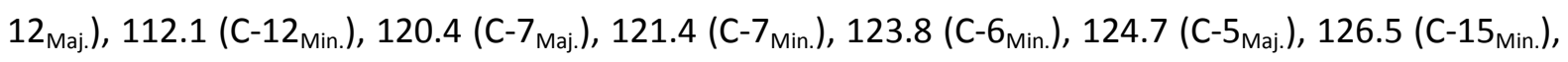

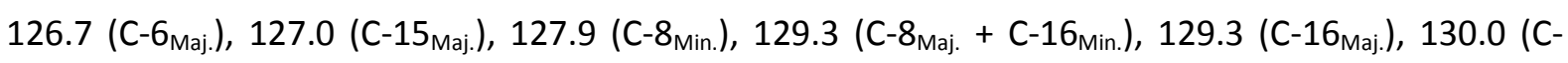

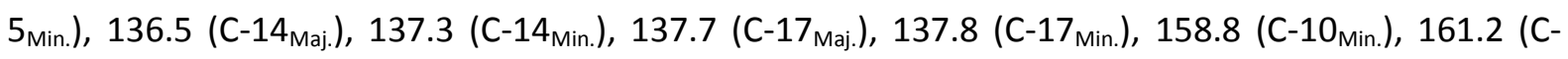

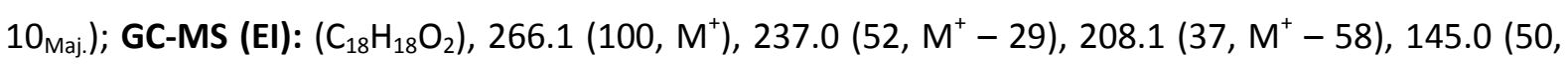
$\left.M^{+}-121\right), 131.0\left(96, M^{+}-135\right), 119.1\left(76, M^{+}-147\right), 105.0\left(68, M^{+}-161\right), 91.1\left(92, M^{+}-176\right), 77.1$ $\left(52, \mathrm{M}^{+}-189\right)$; IR spectrum (neat) $\left(\mathrm{cm}^{-1}\right)=2969,1478,1460,1231,1115,1066,964,929,809,750$; $[\alpha]^{23}{ }_{D}=-70.5\left(c 0.39, \mathrm{CH}_{2} \mathrm{Cl}_{2}\right.$ ); HPLC: OD-H, $204 \mathrm{~nm}$, Hexane:iPrOH, 98:2, $1 \mathrm{~mL} / \mathrm{min}, 30^{\circ} \mathrm{C}, t_{\mathrm{R} 1}=11.6$ and $t_{\mathrm{R} 2}=20.2 \mathrm{~min}$. 
<smiles>Cc1ccc([C@H]2OC3(O)Oc4ccccc4[C@H]3[C@H]2C)cc1</smiles>

$(2 R, 3 R, 3 a S, 8 a R)-3-M e t h y l-2-(p$-tolyl)-2,3,3a,8a-tetrahydrofuro[2,3-b]benzofuran (3o)

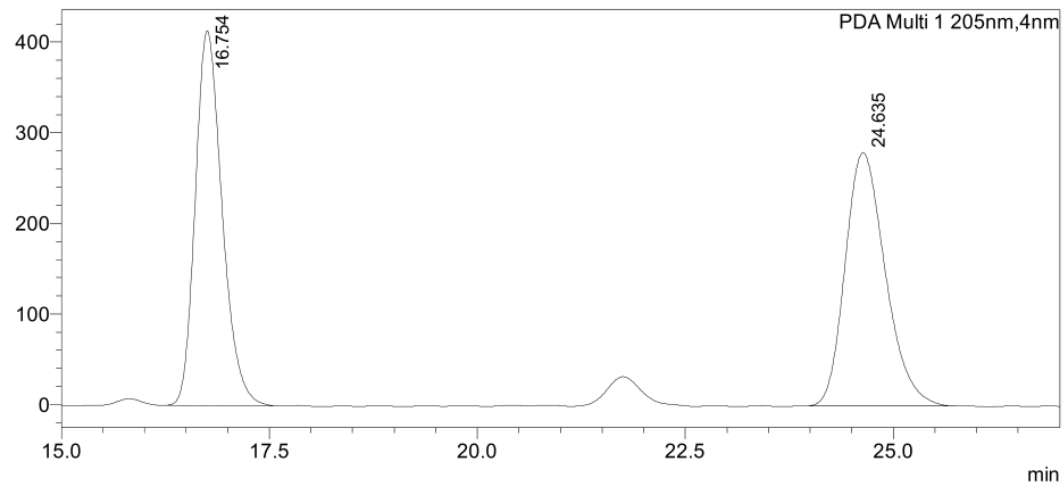

$<$ Peak Table>

\begin{tabular}{l} 
PDA Ch1 205nm \\
Peak\# Ret. Time \\
\hline
\end{tabular}

\begin{tabular}{|r|r|r|}
\hline Peak\# & Ret. Time & Area\% \\
\hline 1 & 16.754 & 49.829 \\
\hline 2 & 24.635 & 50.171 \\
\hline
\end{tabular}

\begin{tabular}{|r|r|r|}
\hline 2 & 24.635 & 50.171 \\
\hline Total & & 100.000 \\
\hline
\end{tabular}

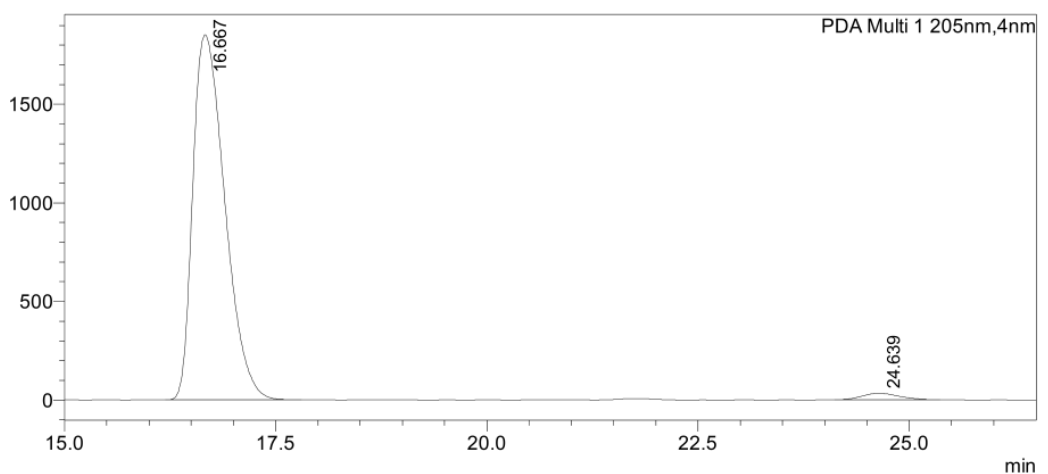

<Peak Table>

PDA Ch1 205nm
Peak\# Ret. Time

\begin{tabular}{|r|r|r|}
\hline 1 & 16.667 & 97.931 \\
\hline 2 & 24.639 & 2.069 \\
\hline
\end{tabular}

\begin{tabular}{l|r|r}
\hline Total & 2.069 \\
\hline
\end{tabular} 
<smiles>COC1(Oc2ccccc2Br)CCCO1</smiles>

\section{2-(2-Bromophenoxy)tetrahydrofuran (4a)}

Prepared according to GP2 using 2,3-dihydrofuran (1a) (76 $\mu \mathrm{L}, 1 \mathrm{mmol}$ ) and 2-bromophenol (2a) (23 $\mu \mathrm{L}, 0.2 \mathrm{mmol}$ ) in absence of $\mathrm{NaOtBu}$. Isolated by column chromatography (Pentane: $\mathrm{Et}_{2} \mathrm{O} 30: 1$ ) as a colorless oil with $\mathrm{R}_{\mathrm{f}}=0.41 ;{ }^{1} \mathrm{H}-\mathrm{NMR}\left(\mathrm{C}_{6} \mathrm{D}_{6}, 400 \mathrm{MHz}\right): \delta(\mathrm{ppm})=1.30(\mathrm{~m}, 1 \mathrm{H}, \mathrm{H}-4), 1.50(\mathrm{~m}, 1 \mathrm{H}, \mathrm{H}-3)$, $1.77\left(\mathrm{~m}, 1 \mathrm{H}, \mathrm{H}-4^{\prime}\right), 2.02\left(\mathrm{~m}, 1 \mathrm{H}, \mathrm{H}-3^{\prime}\right), 3.54(\mathrm{~m}, 1 \mathrm{H}, \mathrm{H}-5), 3.77\left(\mathrm{~m}, 1 \mathrm{H}, \mathrm{H}-5^{\prime}\right), 5.46\left(\mathrm{dd},{ }^{3} J_{\mathrm{H}-\mathrm{H}}=5.0 \mathrm{~Hz}\right.$, $\left.{ }^{3} J_{\mathrm{H}-\mathrm{H}}=1.0 \mathrm{~Hz}, 1 \mathrm{H}, \mathrm{H}-2\right), 6.51\left(\mathrm{dt},{ }^{3} \mathrm{~J}_{\mathrm{H}-\mathrm{H}}=7.7 \mathrm{~Hz},{ }^{4} J_{\mathrm{H}-\mathrm{H}}=1.5 \mathrm{~Hz}, 1 \mathrm{H}, \mathrm{H}-9\right), 6.93$ (ddd, ${ }^{3} J_{\mathrm{H}-\mathrm{H}}=8.6 \mathrm{~Hz},{ }^{3} J_{\mathrm{H}-\mathrm{H}}=$ $\left.7.5 \mathrm{~Hz},{ }^{4} J_{\mathrm{H}-\mathrm{H}}=1.6 \mathrm{~Hz}, 1 \mathrm{H}, \mathrm{H}-10\right), 7.22\left(\mathrm{dd},{ }^{3} J_{\mathrm{H}-\mathrm{H}}=8.2 \mathrm{~Hz},{ }^{4} J_{\mathrm{H}-\mathrm{H}}=1.5 \mathrm{~Hz}, 1 \mathrm{H}, \mathrm{H}-11\right), 7.42\left(\mathrm{dd},{ }^{3} J_{\mathrm{H}-\mathrm{H}}=7.9\right.$ $\left.\mathrm{Hz},{ }^{4} J_{-H}=1.6 \mathrm{~Hz}, 1 \mathrm{H}, \mathrm{H}-8\right) ;{ }^{13} \mathrm{C}\left\{{ }^{1} \mathrm{H}\right\}-\mathrm{NMR}\left(\mathrm{C}_{6} \mathrm{D}_{6}, 100 \mathrm{MHz}\right): \delta(\mathrm{ppm})=23.4$ (C-4), 32.9 (C-3), $68.1(\mathrm{C}-5)$, 103.5 (C-2), 113.7 (C-7), 117.3 (C-11), 122.9 (C-9), 128.5 (C-10), 133.5 (C-8), 154.66 (C-6); IR spectrum (neat) $\left(\mathrm{cm}^{-1}\right)=2956,1587,1475,1441,1362,1275,1237,1179,1075,1030,960,917,871$, 745. 


\section{NMR spectra}

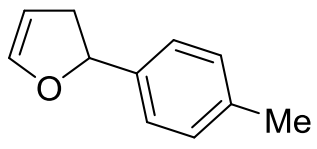

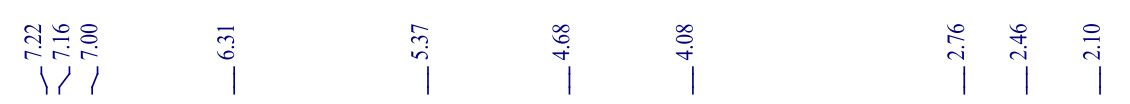

(1c)

${ }^{1} \mathrm{H}-\mathrm{NMR}$

$\mathrm{C}_{6} \mathrm{D}_{6}, 400 \mathrm{MHz}, 298 \mathrm{~K}$

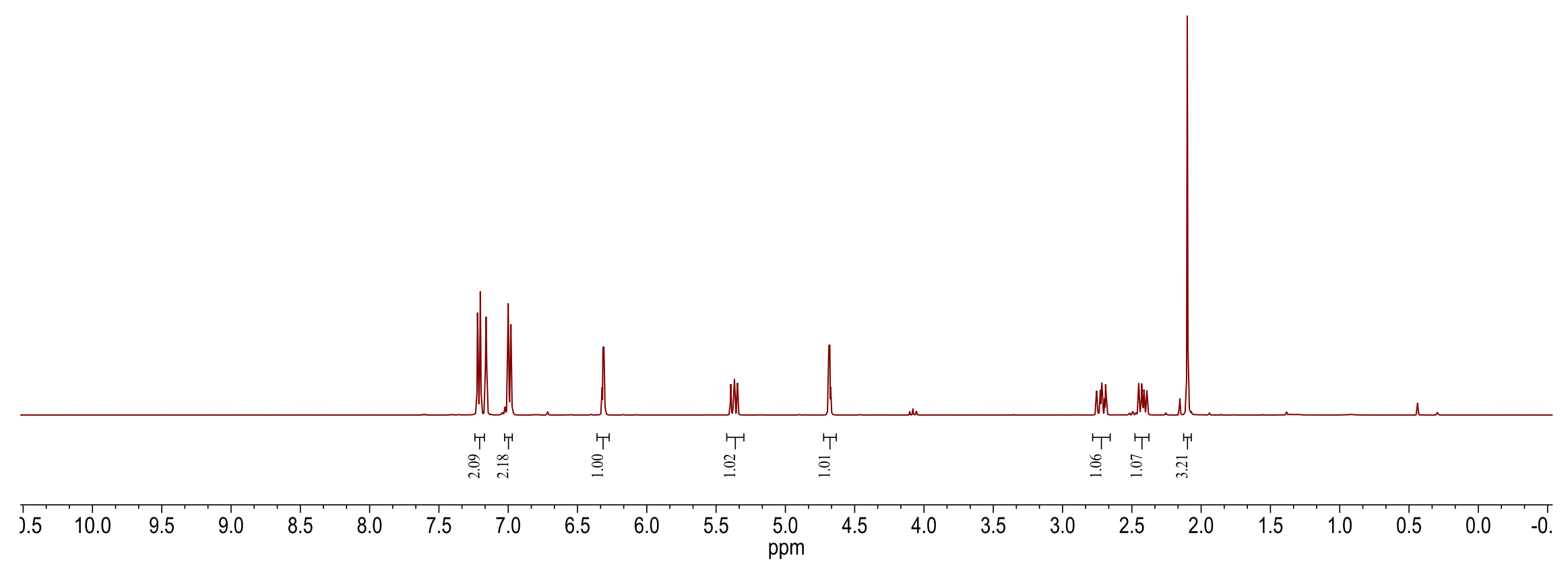




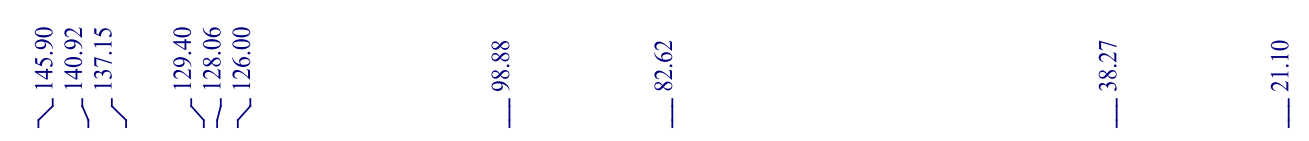

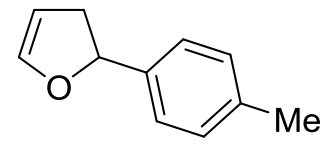

(1c)

${ }^{13} \mathrm{C}\left\{{ }^{1} \mathrm{H}\right\}-\mathrm{NMR}$

$\mathrm{C}_{6} \mathrm{D}_{6}, 100 \mathrm{MHz}, 298 \mathrm{~K}$

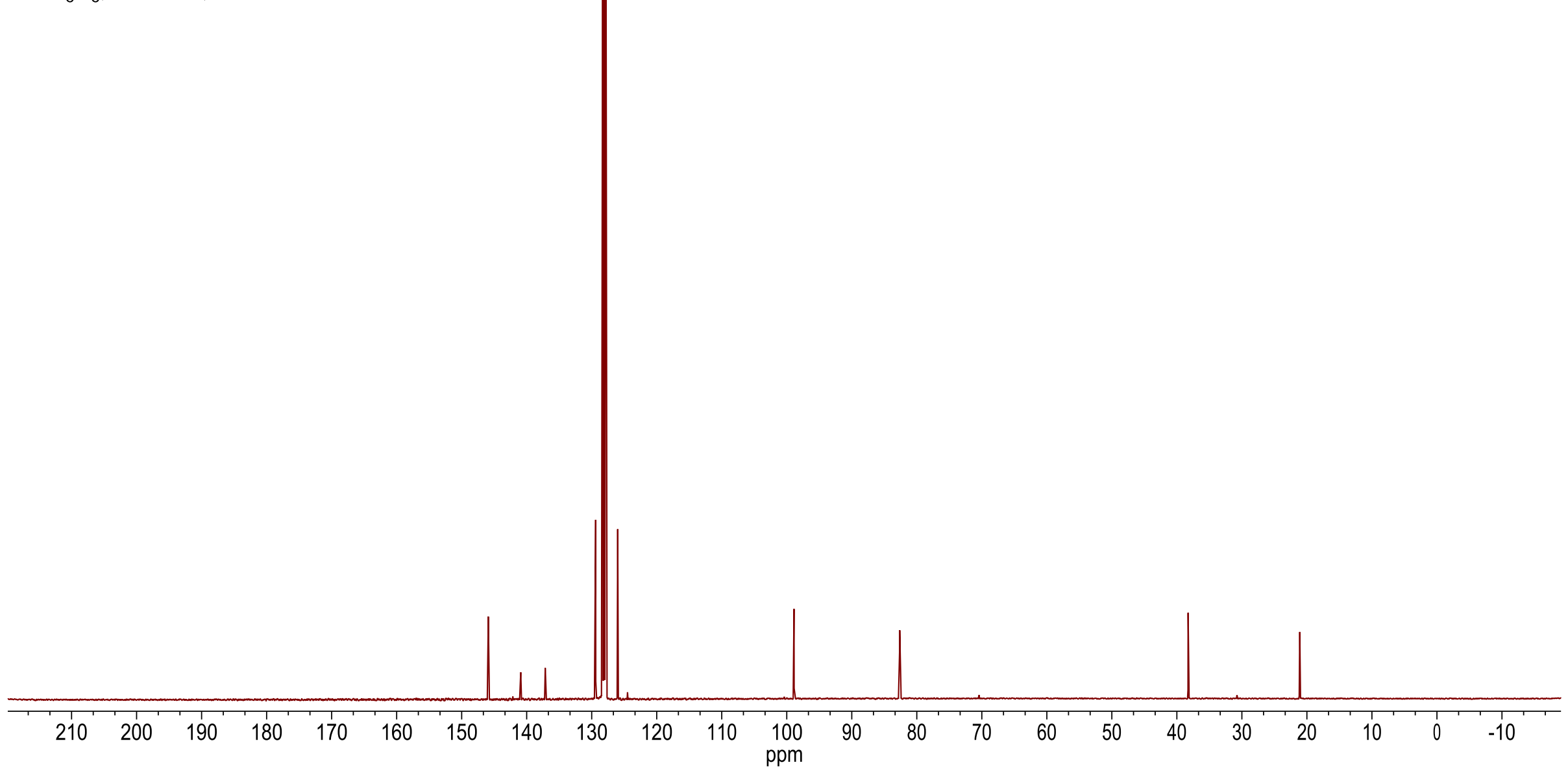




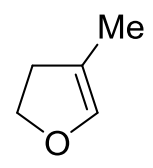

\begin{tabular}{|c|c|}
\hline$\stackrel{?}{1}$ & $\ddot{b}$ \\
\hline
\end{tabular}

${ }^{1} \mathrm{H}-\mathrm{NMR}$

$\mathrm{C}_{6} \mathrm{D}_{6}, 400 \mathrm{MHz}, 298 \mathrm{~K}$

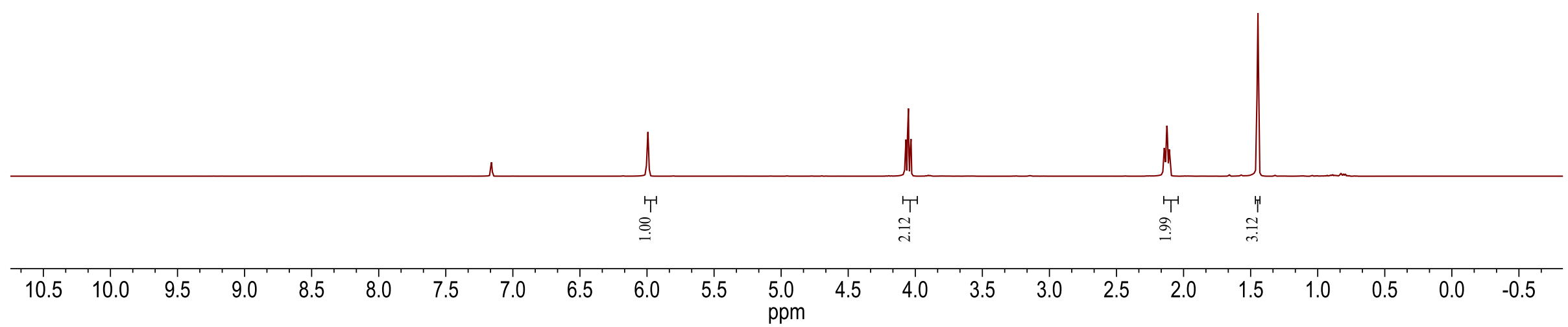




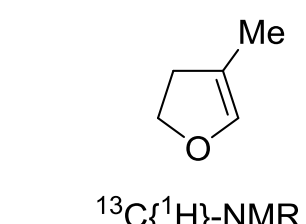

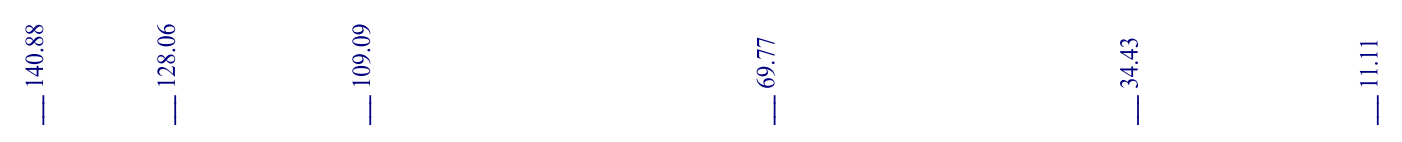

${ }^{13} \mathrm{C}\left\{{ }^{1} \mathrm{H}\right\}-N M R$

$\mathrm{C}_{6} \mathrm{D}_{6}, 100 \mathrm{MHz}, 298 \mathrm{~K}$

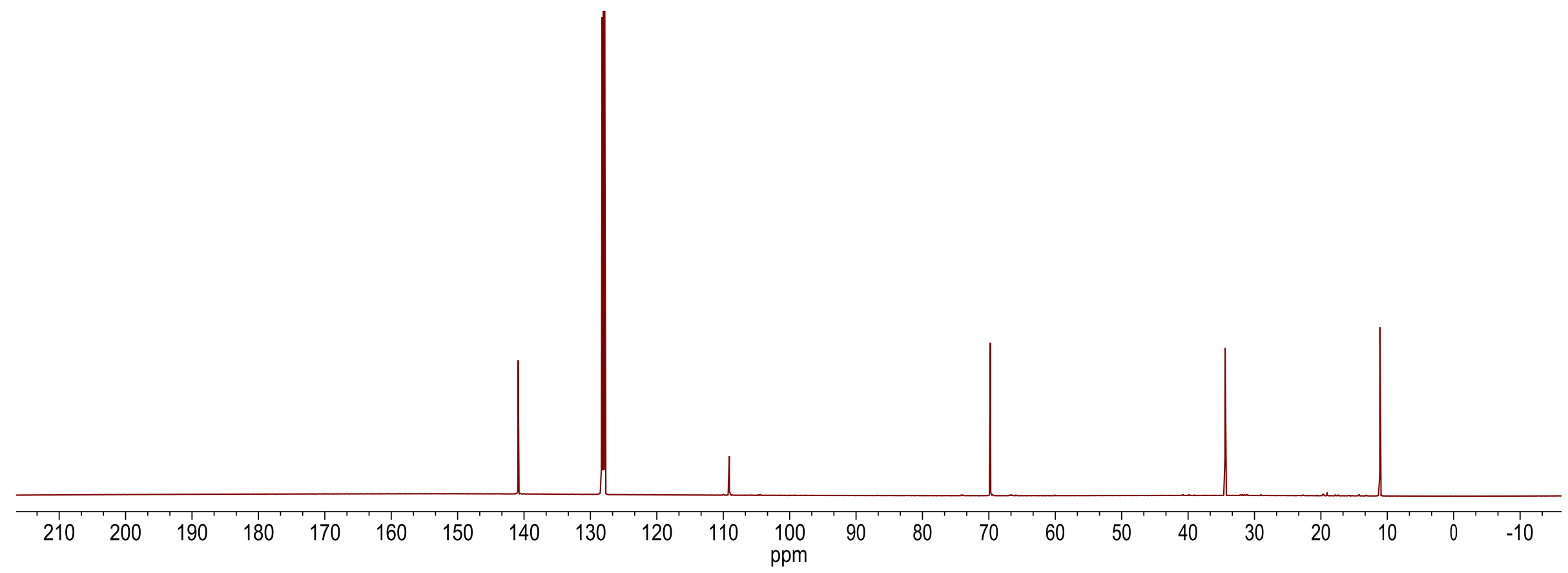




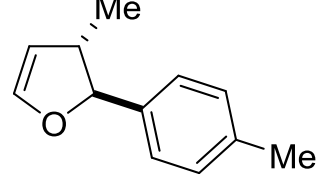

(1d)

${ }^{1} \mathrm{H}-\mathrm{NMR}$

$\mathrm{C}_{6} \mathrm{D}_{6}, 400 \mathrm{MHz}, 298 \mathrm{~K}$

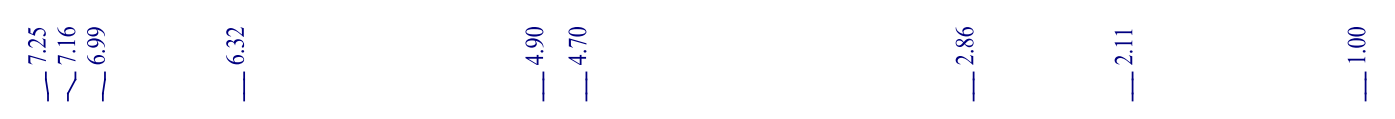




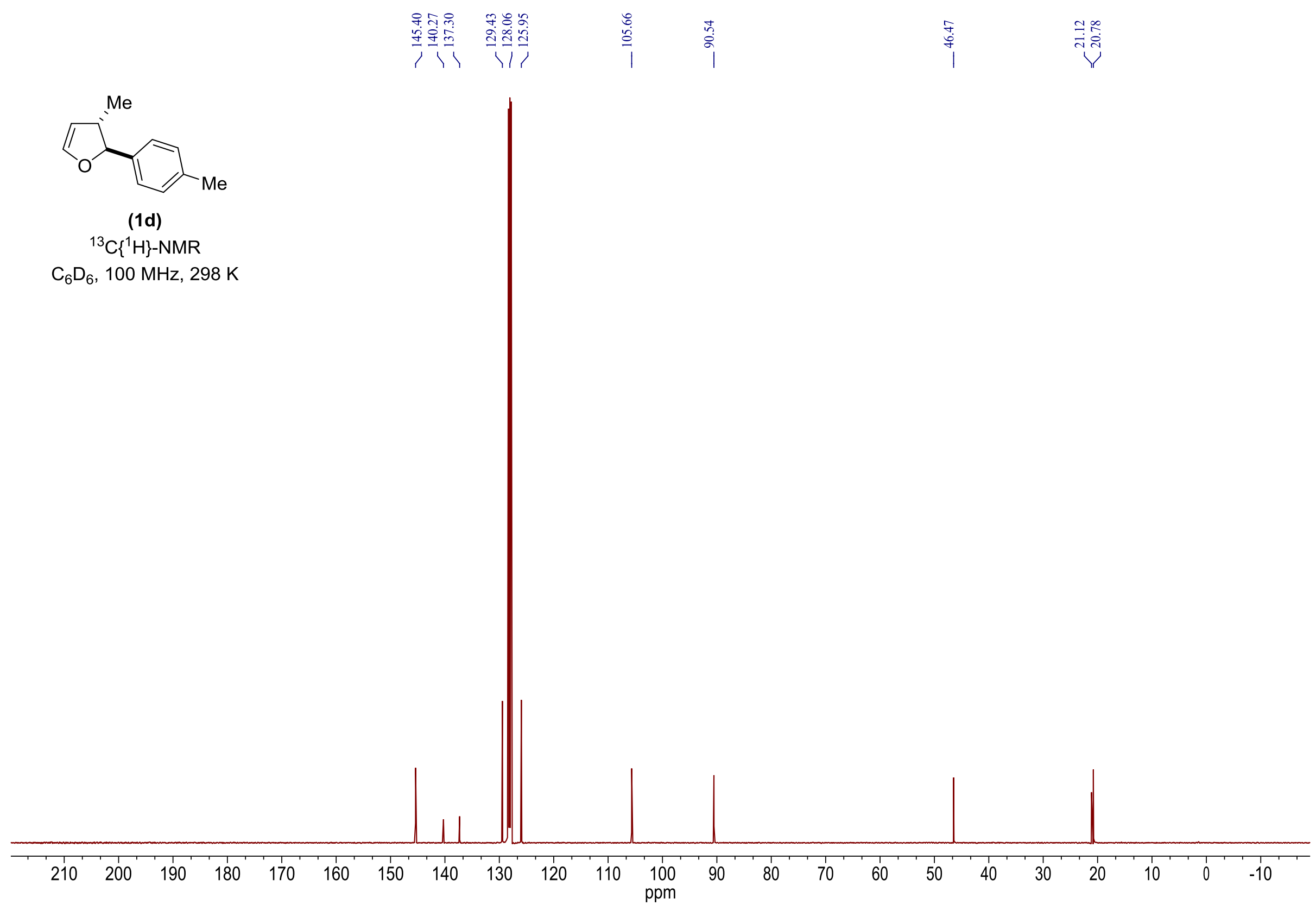




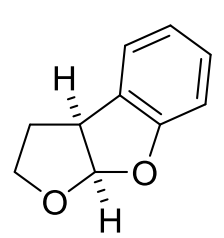

$$
\text { }
$$

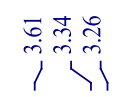

$\stackrel{1}{\circ}$

(3a)

${ }^{1} \mathrm{H}-\mathrm{NMR}$

$\mathrm{C}_{6} \mathrm{D}_{6}, 400 \mathrm{MHz}, 298 \mathrm{~K}$

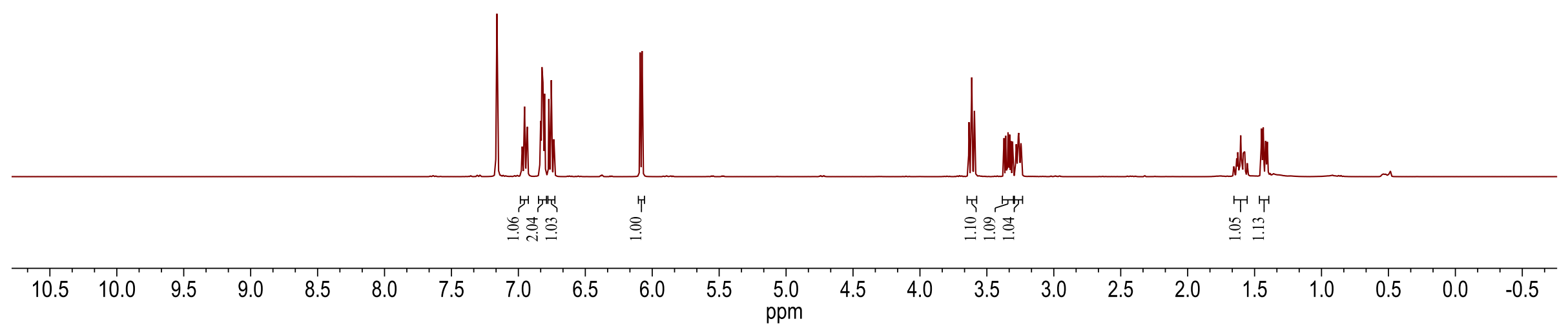



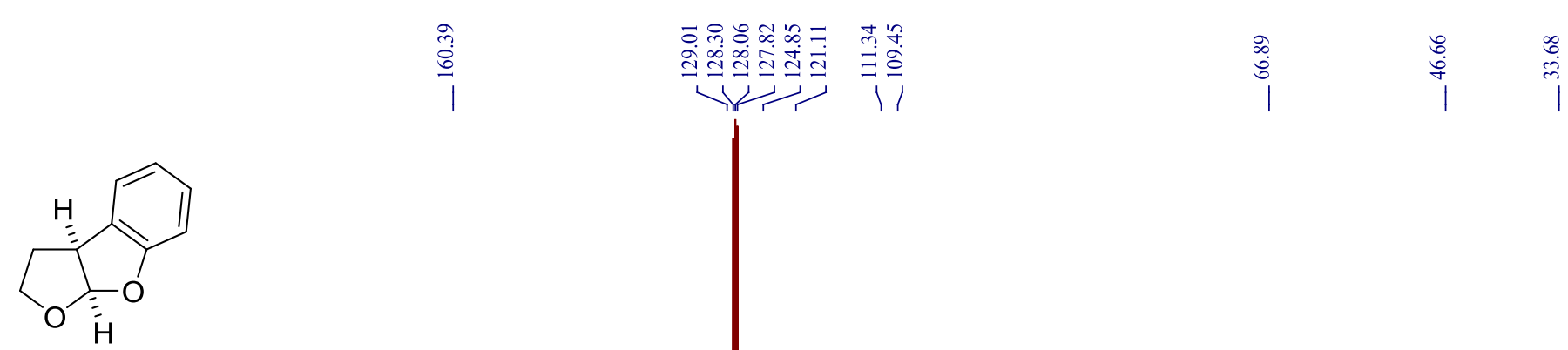

(3a)

${ }^{13} \mathrm{C}\left\{{ }^{1} \mathrm{H}\right\}-\mathrm{NMR}$

$\mathrm{C}_{6} \mathrm{D}_{6}, 100 \mathrm{MHz}, 298 \mathrm{~K}$

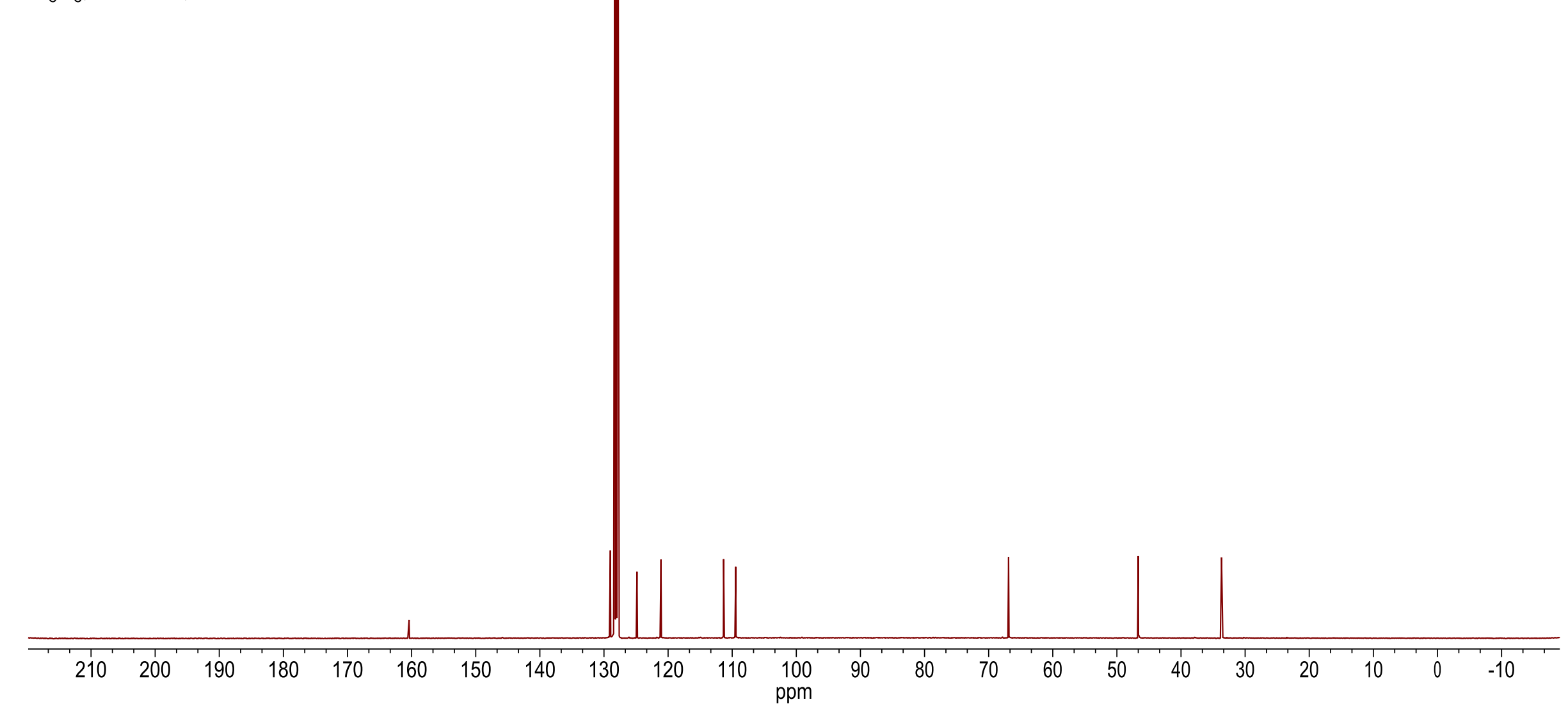




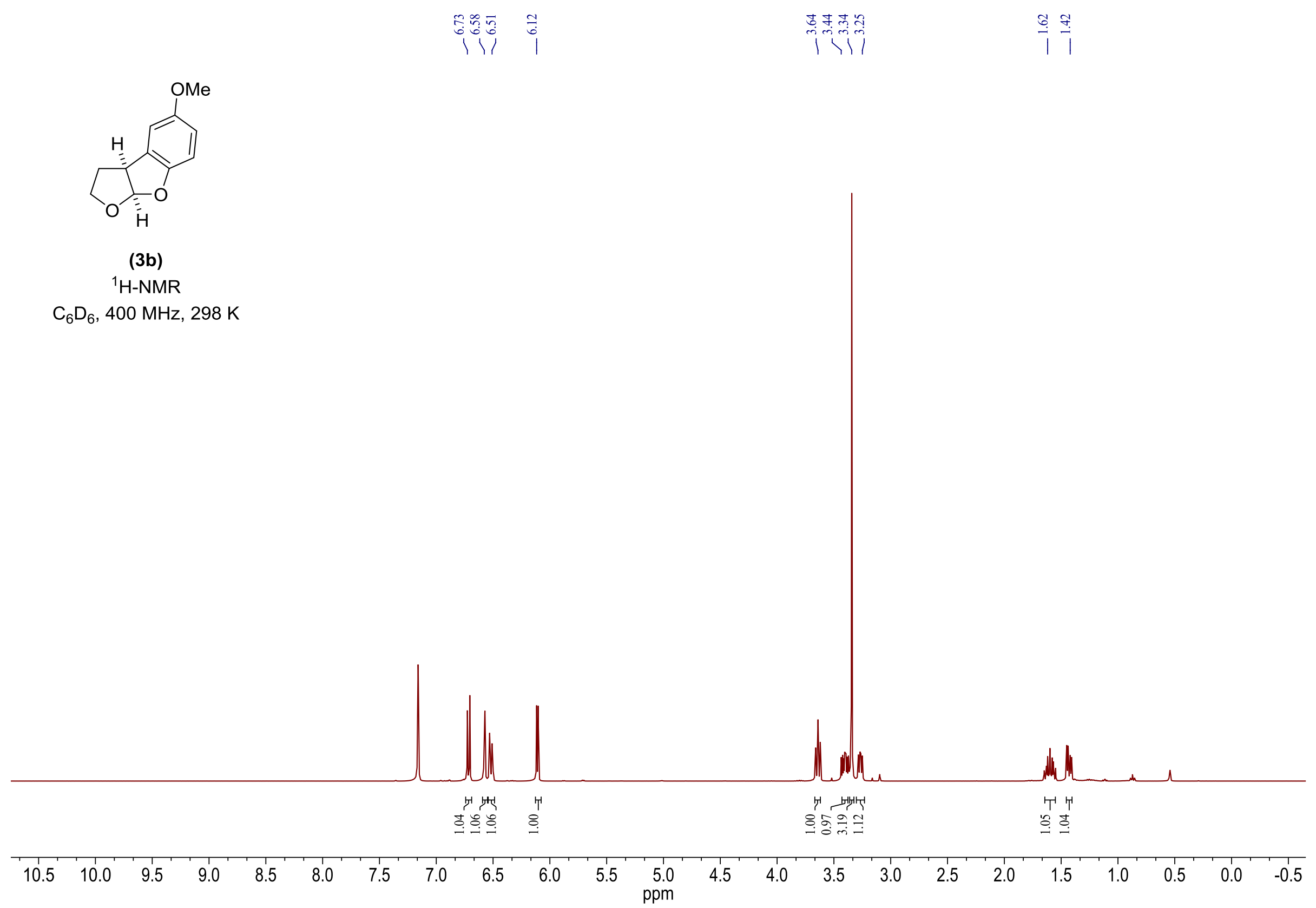




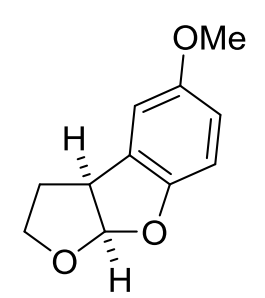

(3b)

${ }^{13} \mathrm{C}\left\{{ }^{1} \mathrm{H}\right\}-N M R$

$\mathrm{C}_{6} \mathrm{D}_{6}, 100 \mathrm{MHz}, 298 \mathrm{~K}$

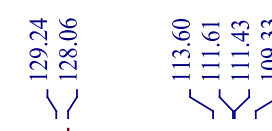

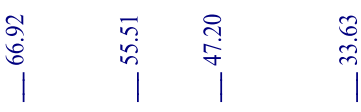

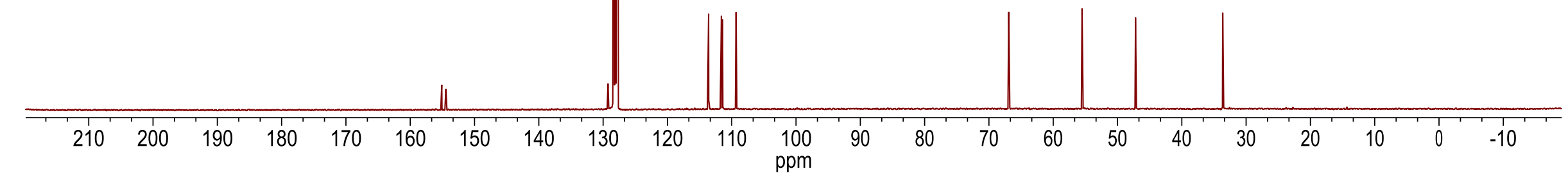




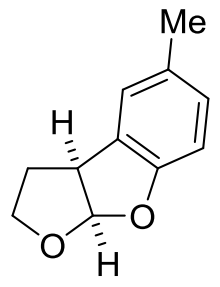

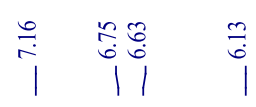

țiji

$\stackrel{1}{7}$

(3c)

${ }^{1} \mathrm{H}-\mathrm{NMR}$

$\mathrm{C}_{6} \mathrm{D}_{6}, 400 \mathrm{MHz}, 298 \mathrm{~K}$

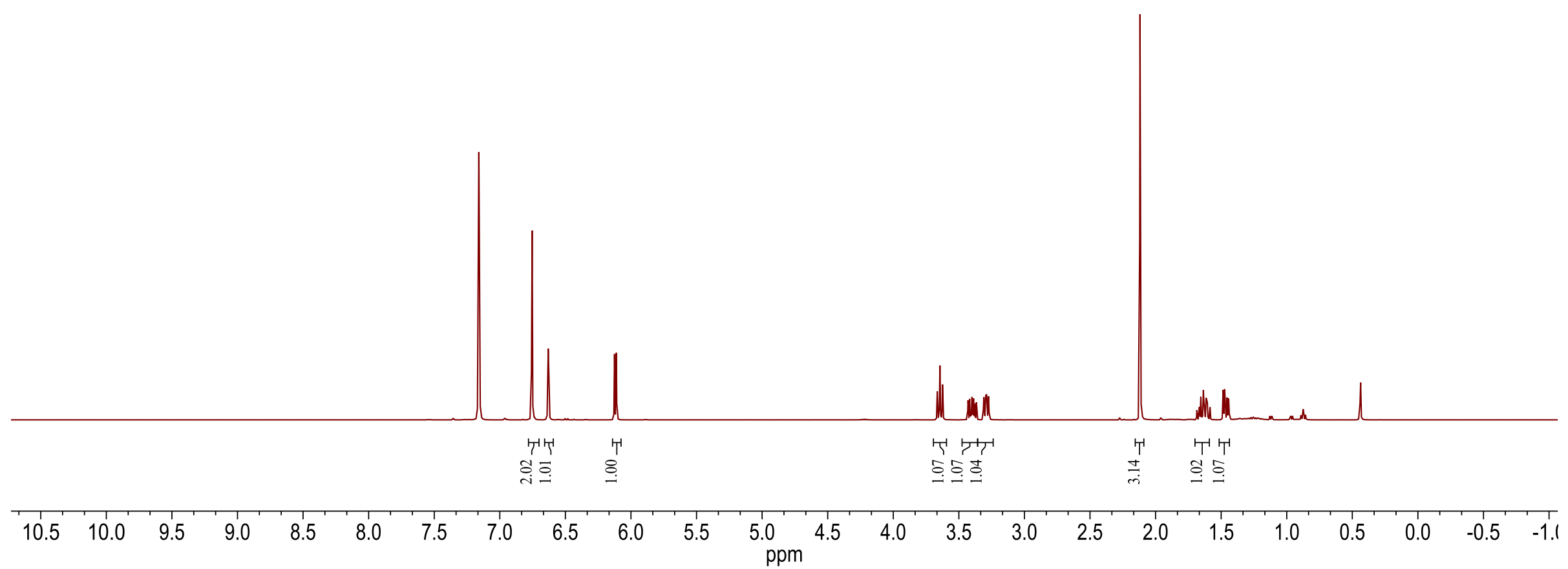




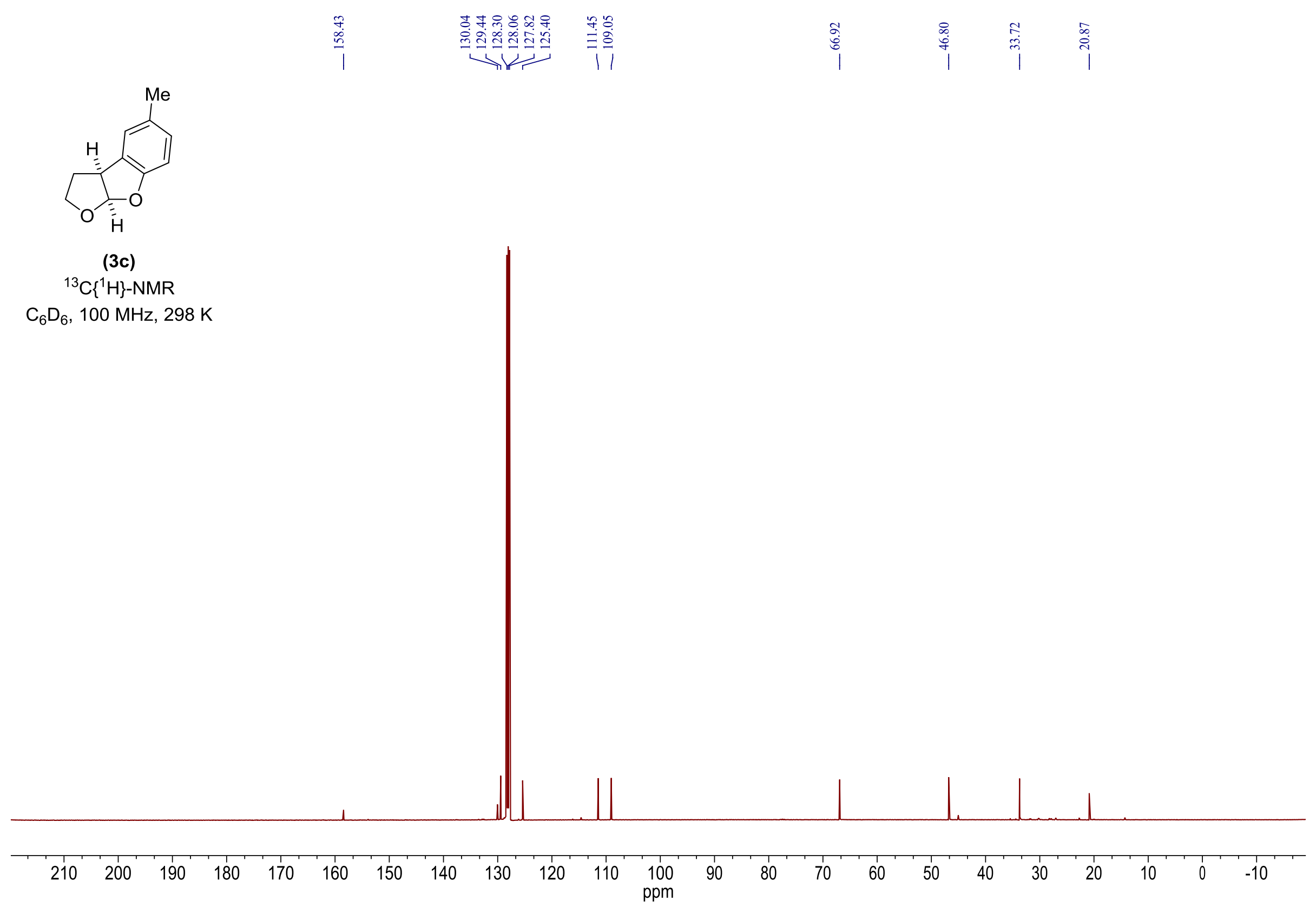



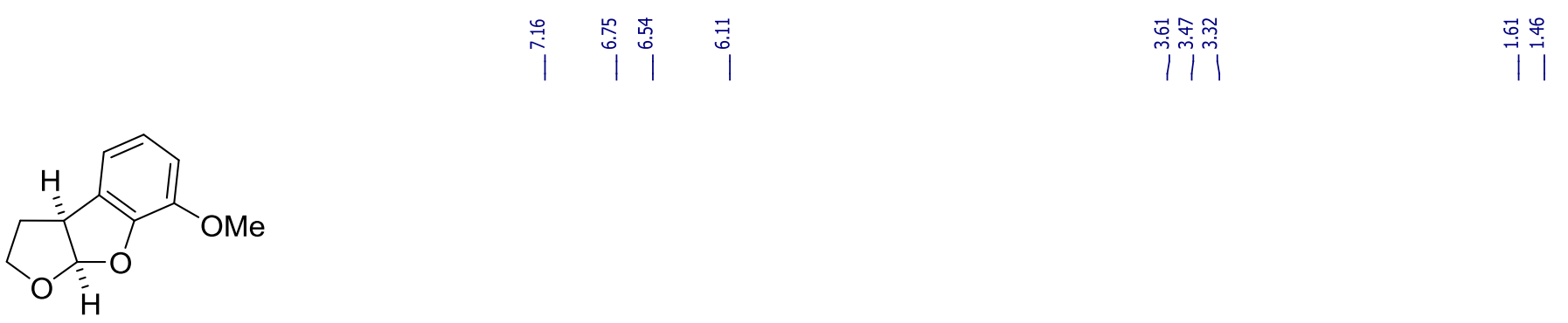

(3d)

${ }^{1} \mathrm{H}-\mathrm{NMR}$

$\mathrm{C}_{6} \mathrm{D}_{6}, 400 \mathrm{MHz}, 298 \mathrm{~K}$

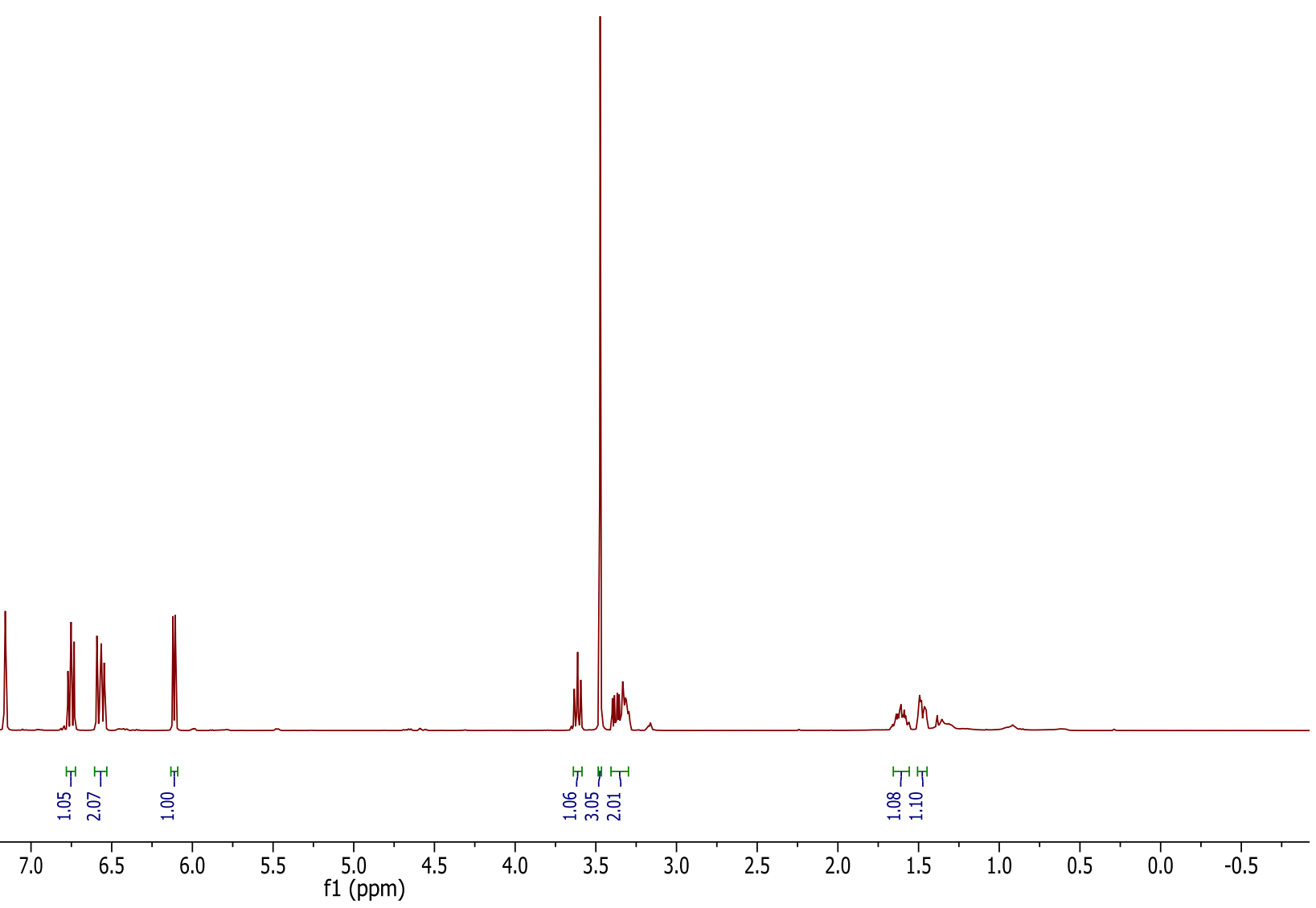



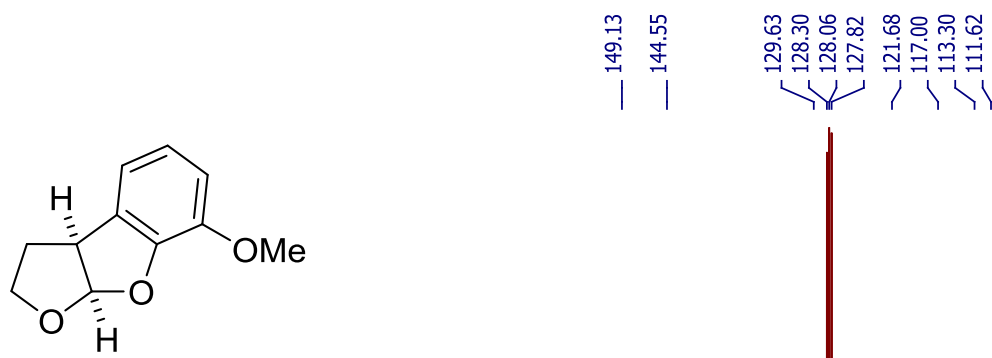

|

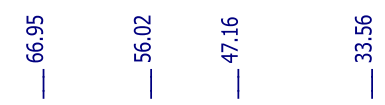

(3d)

${ }^{13} \mathrm{C}\left\{{ }^{1} \mathrm{H}\right\}-\mathrm{NMR}$

$\mathrm{C}_{6} \mathrm{D}_{6}, 100 \mathrm{MHz}, 298 \mathrm{~K}$

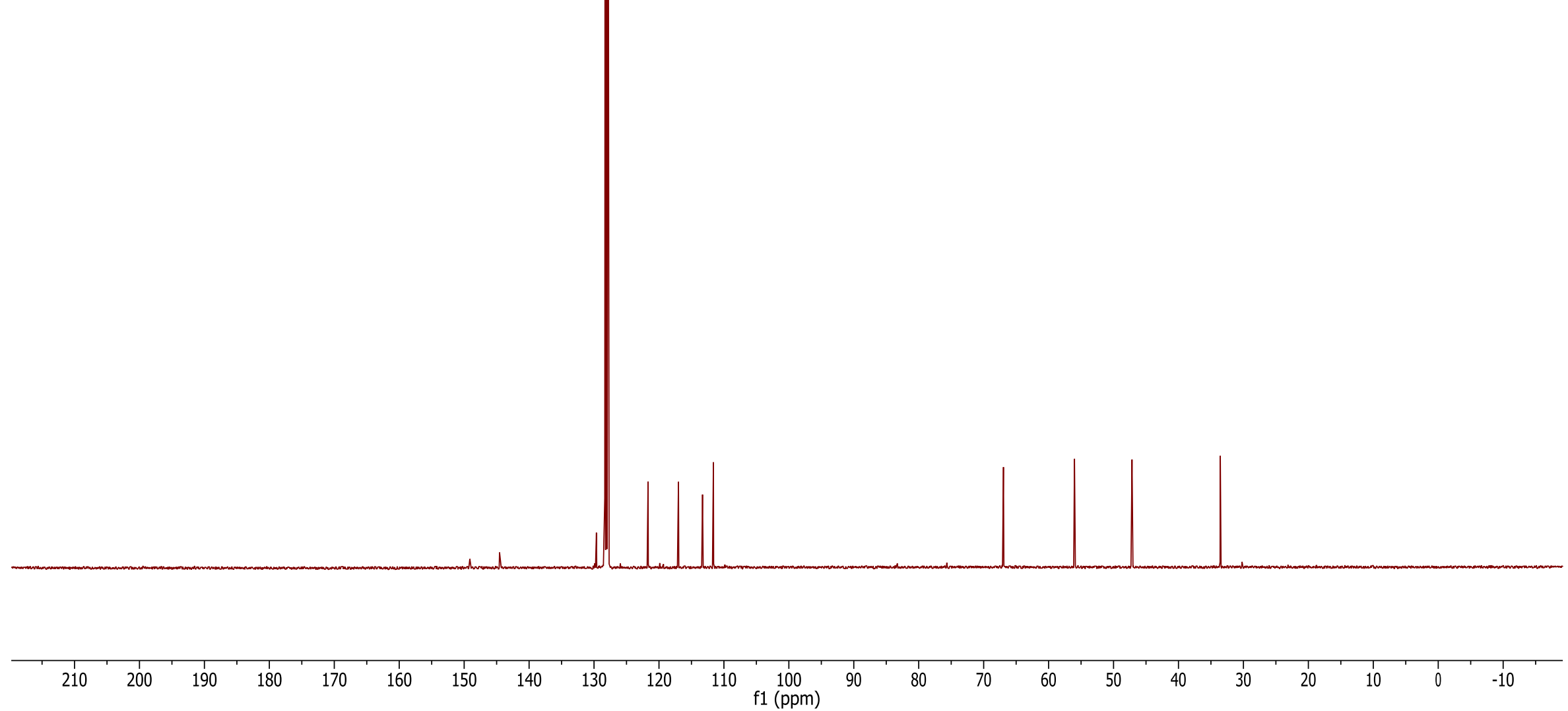



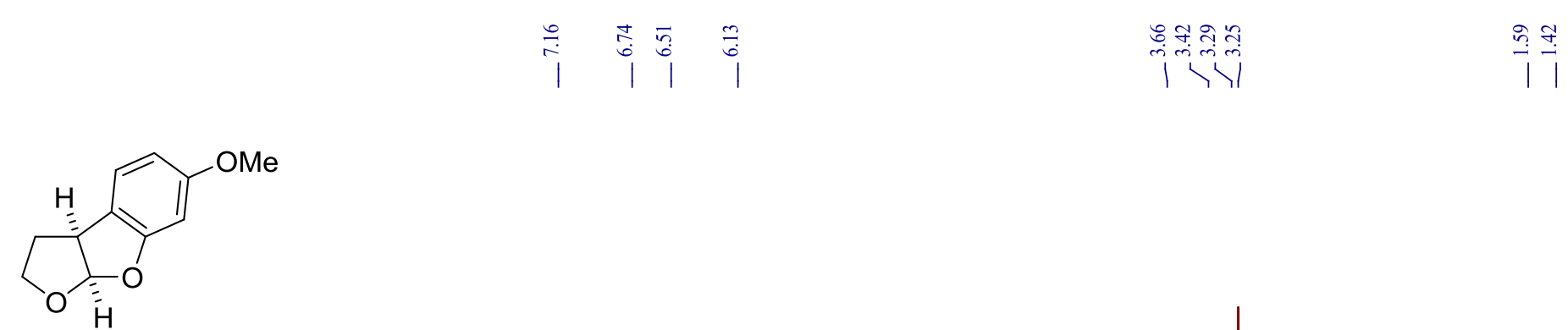

(3e)

${ }^{1} \mathrm{H}-\mathrm{NMR}$

$\mathrm{C}_{6} \mathrm{D}_{6}, 400 \mathrm{MHz}, 298 \mathrm{~K}$

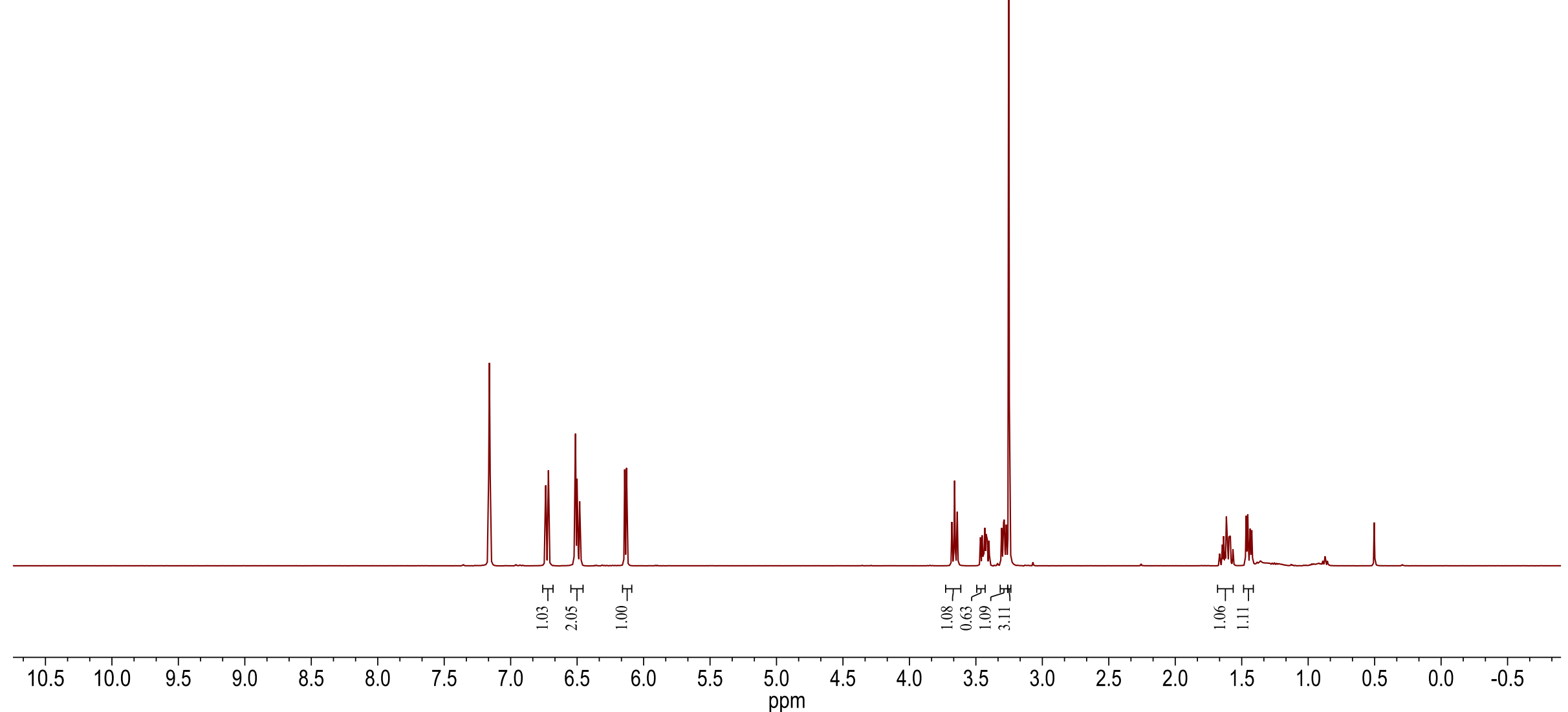




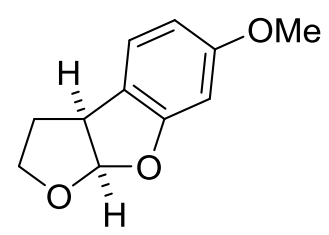

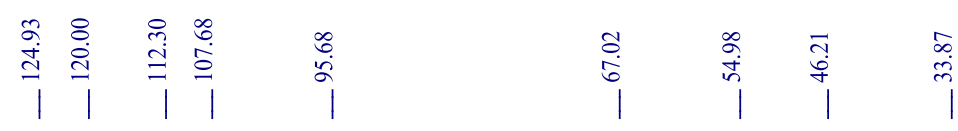

(3e)

${ }^{13} \mathrm{C}\left\{{ }^{1} \mathrm{H}\right\}-N M R$

$\mathrm{C}_{6} \mathrm{D}_{6}, 100 \mathrm{MHz}, 298 \mathrm{~K}$

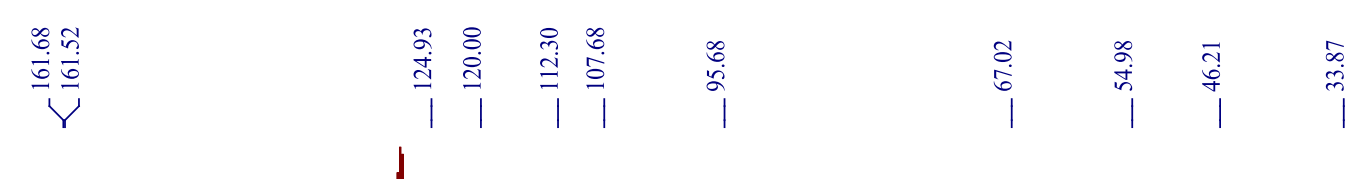

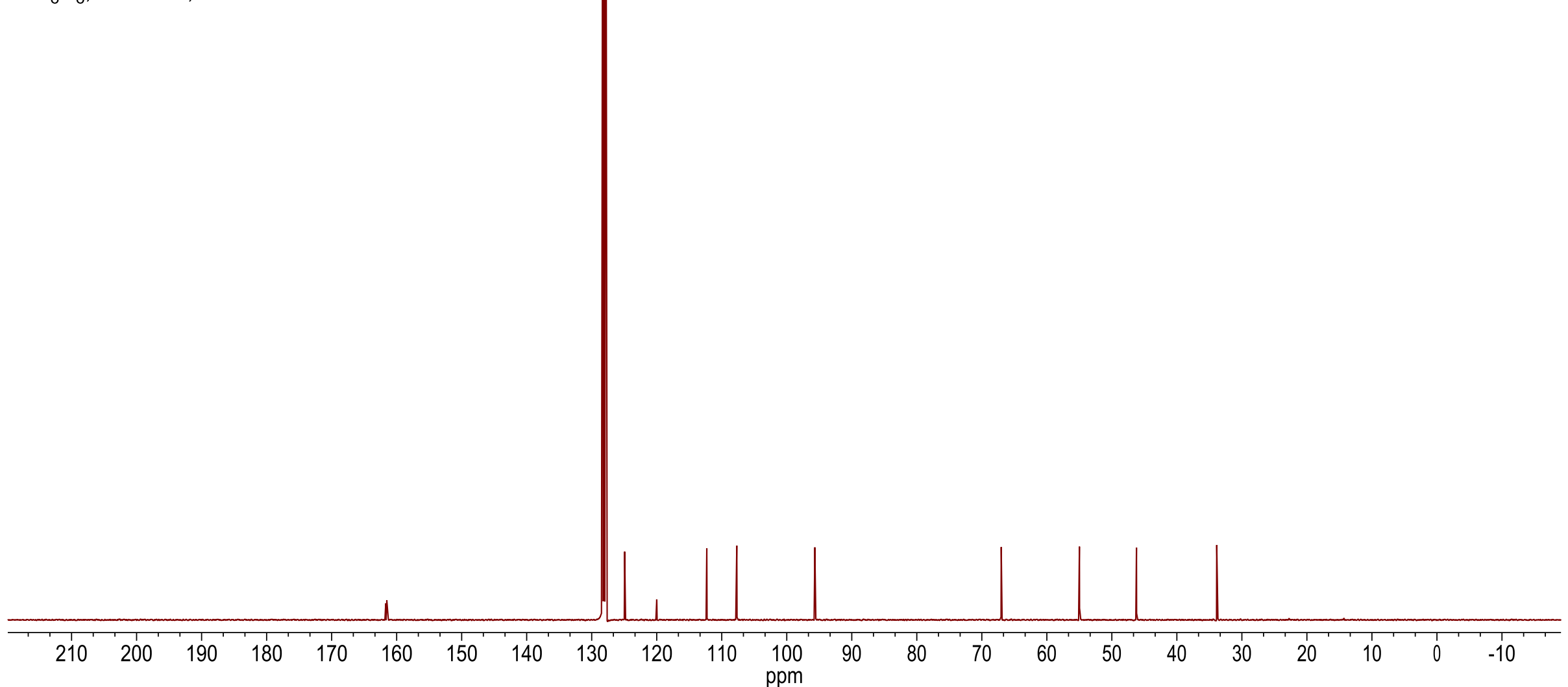



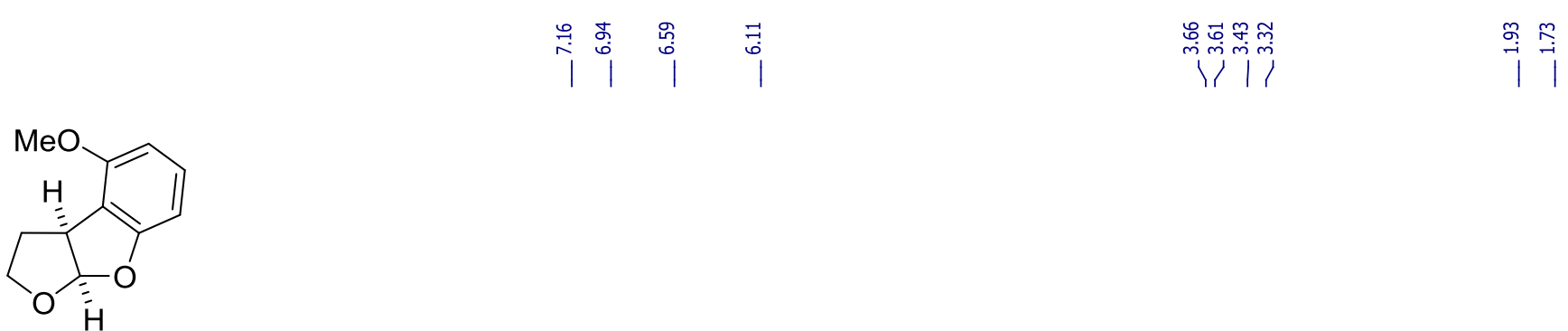

(3f)

${ }^{1} \mathrm{H}-\mathrm{NMR}$

$\mathrm{C}_{6} \mathrm{D}_{6}, 400 \mathrm{MHz}, 298 \mathrm{~K}$

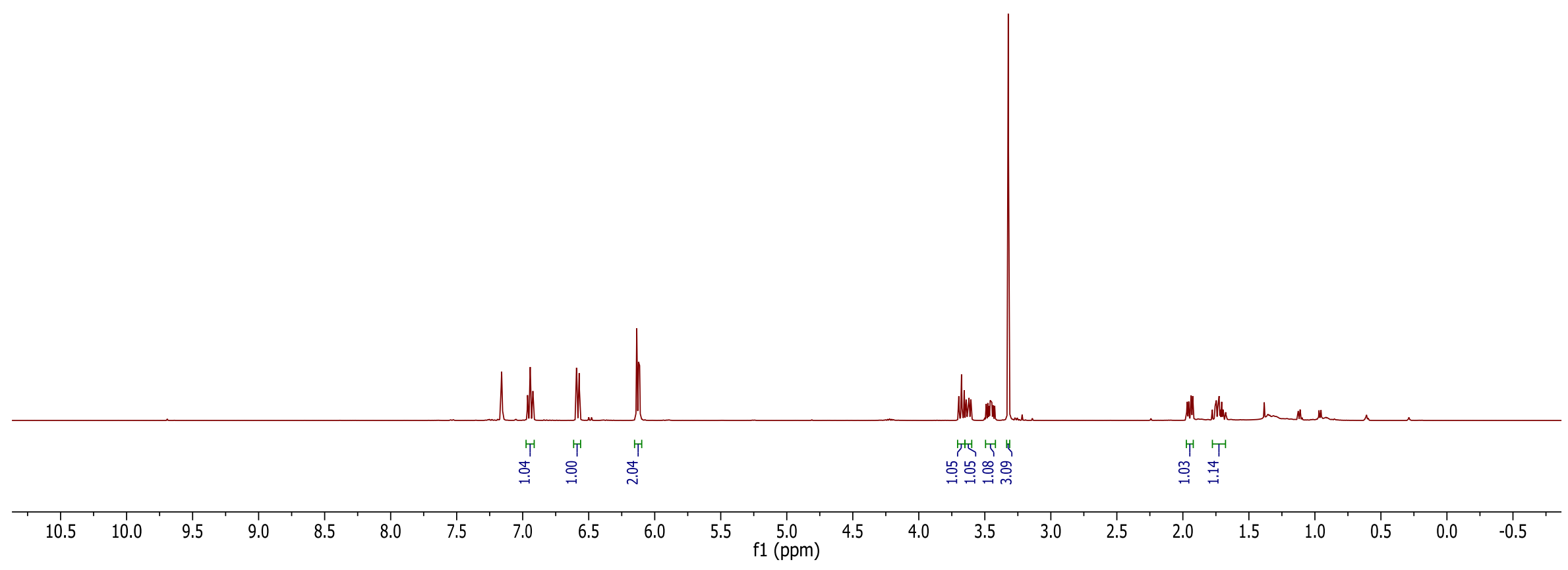




$\mathrm{MeO}$

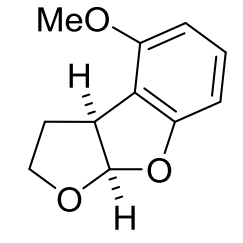

(3f)

${ }^{13} \mathrm{C}\left\{{ }^{1} \mathrm{H}\right\}-N M R$

$\mathrm{C}_{6} \mathrm{D}_{6}, 100 \mathrm{MHz}, 298 \mathrm{~K}$

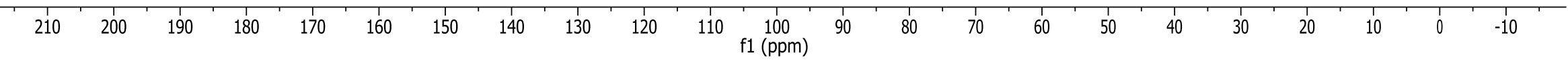




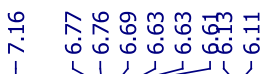

Vili

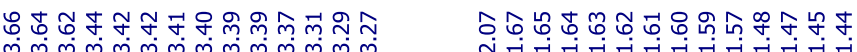

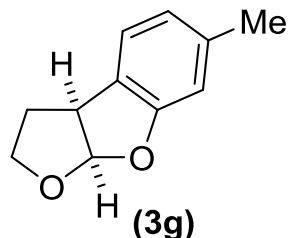

H-NMR

$\mathrm{C}_{6} \mathrm{D}_{6}, 400 \mathrm{MHz}, 298 \mathrm{~K}$

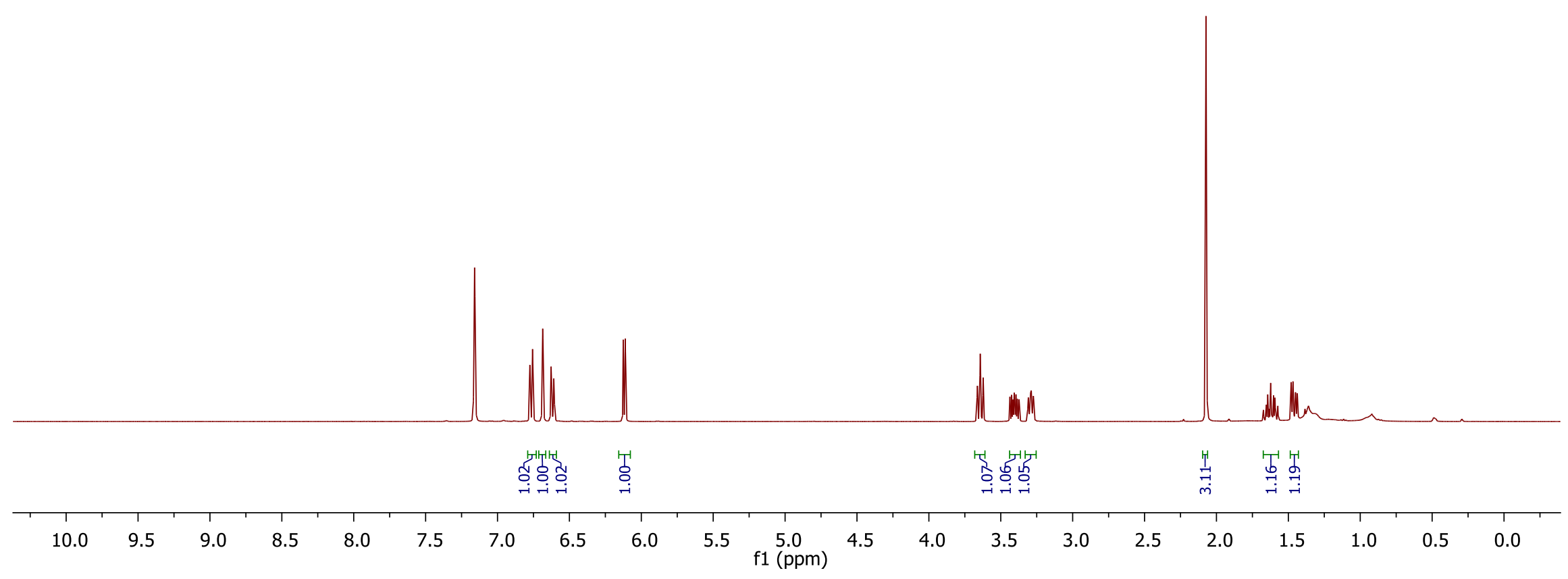




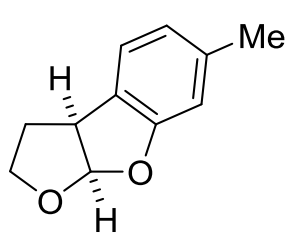

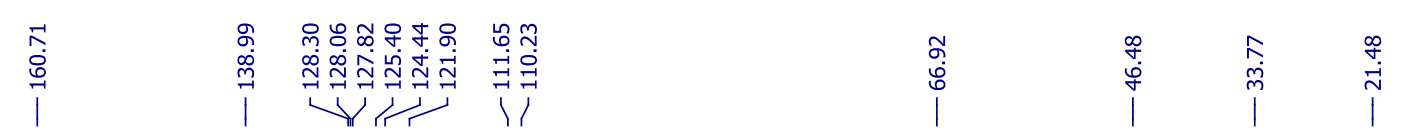

(3g)

${ }^{13} \mathrm{C}\left\{{ }^{1} \mathrm{H}\right\}-\mathrm{NMR}$

$\mathrm{C}_{6} \mathrm{D}_{6}, 100 \mathrm{MHz}, 298 \mathrm{~K}$

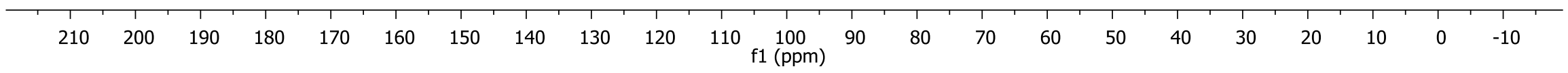




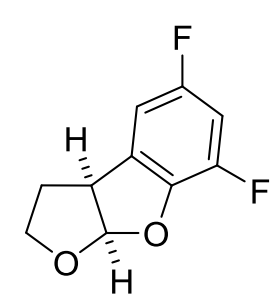

(3h)

$\mathrm{C}_{6} \mathrm{D}_{6}, 400 \mathrm{MHz}, 298 \mathrm{~K}$

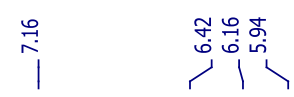

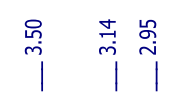

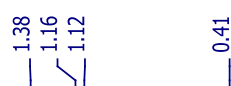

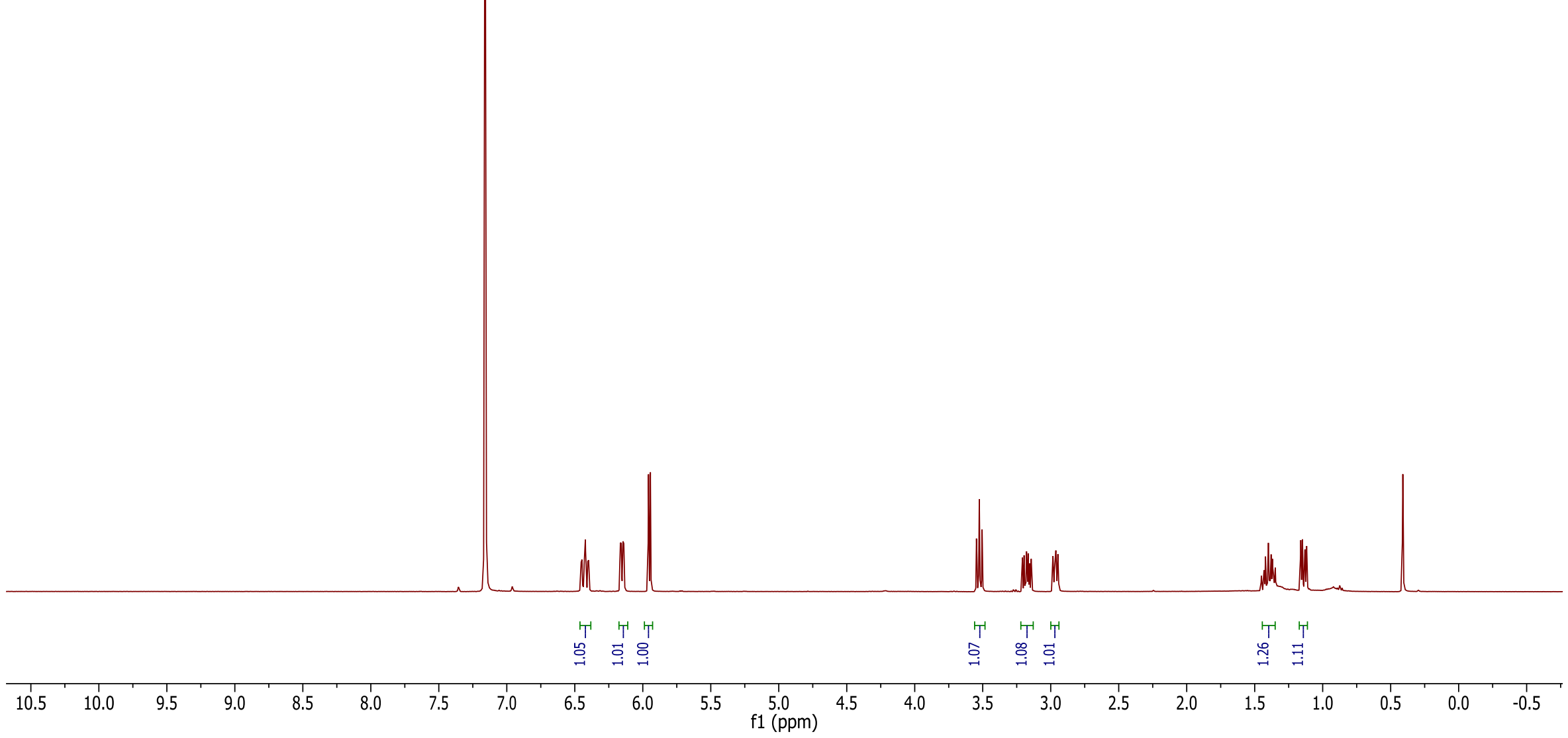




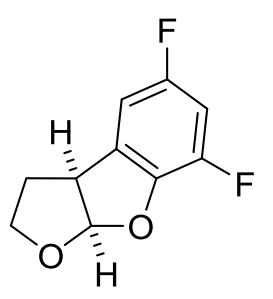

(3h)

${ }^{13} \mathrm{C}\left\{{ }^{1} \mathrm{H}\right\}-\mathrm{NMR}$

$\mathrm{C}_{6} \mathrm{D}_{6}, 100 \mathrm{MHz}, 298 \mathrm{~K}$

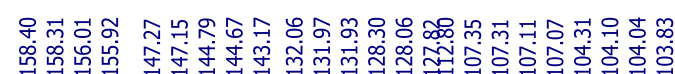

vif

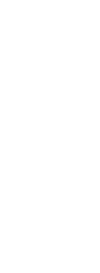

究

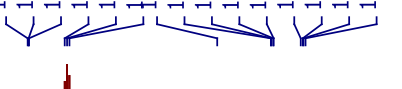

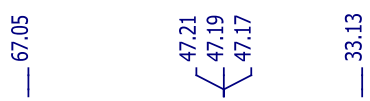

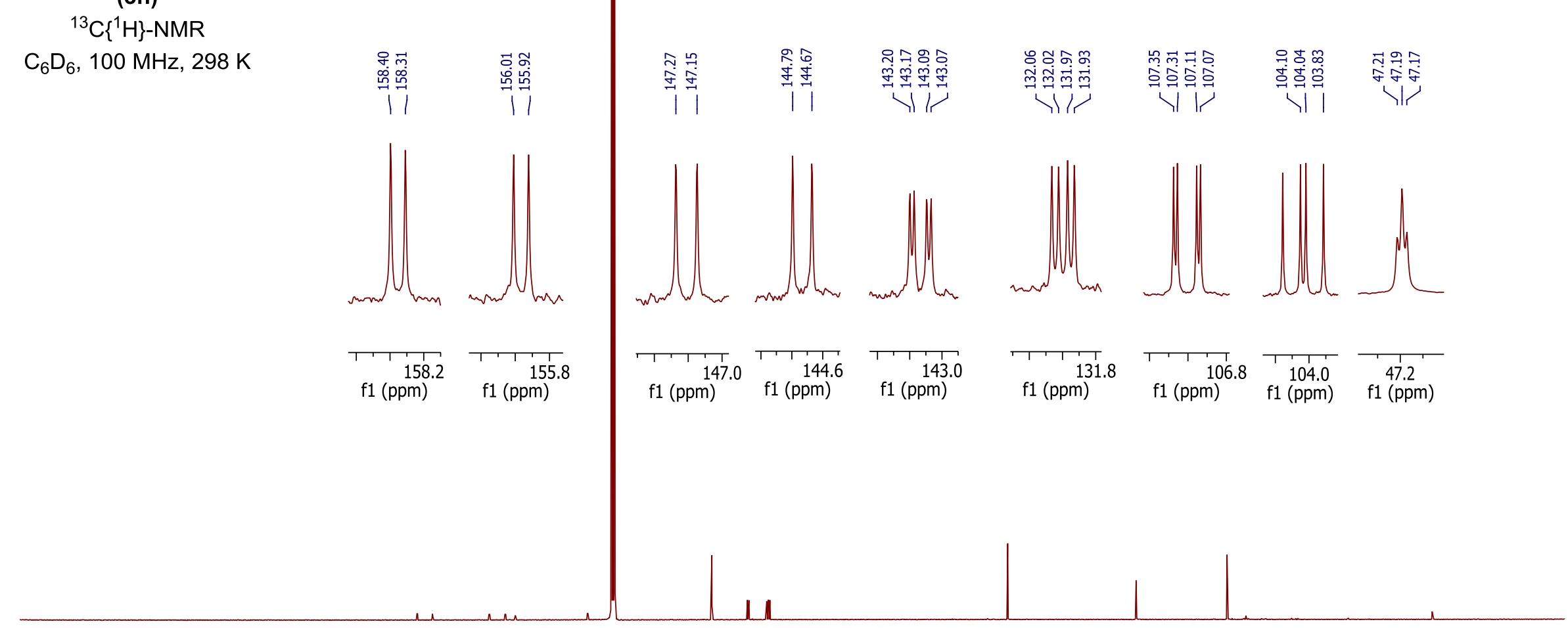




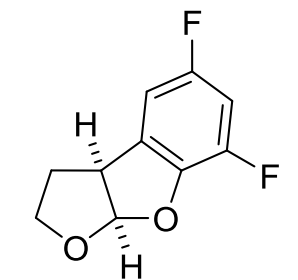

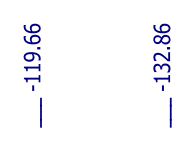

(3h)

${ }^{19}$ F-NMR

$\mathrm{C}_{6} \mathrm{D}_{6}, 282 \mathrm{MHz}, 298 \mathrm{~K}$

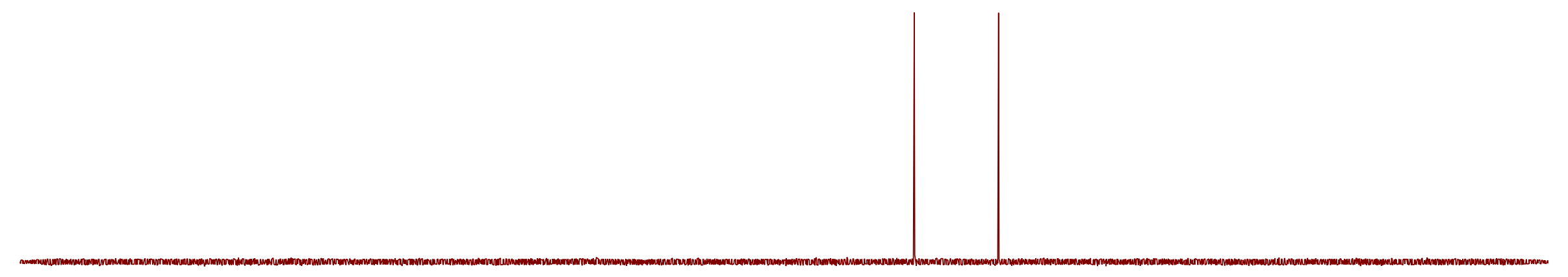

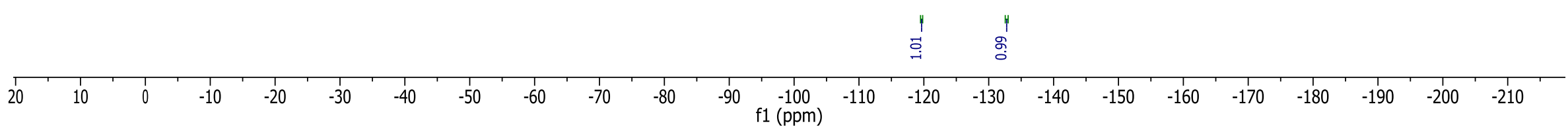




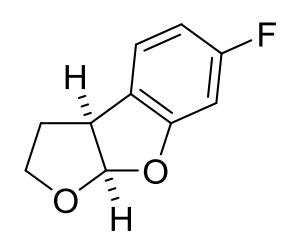

(3i)

$$
{ }^{1} \mathrm{H}-\mathrm{NMR}
$$

$\mathrm{C}_{6} \mathrm{D}_{6}, 400 \mathrm{MHz}, 298 \mathrm{~K}$

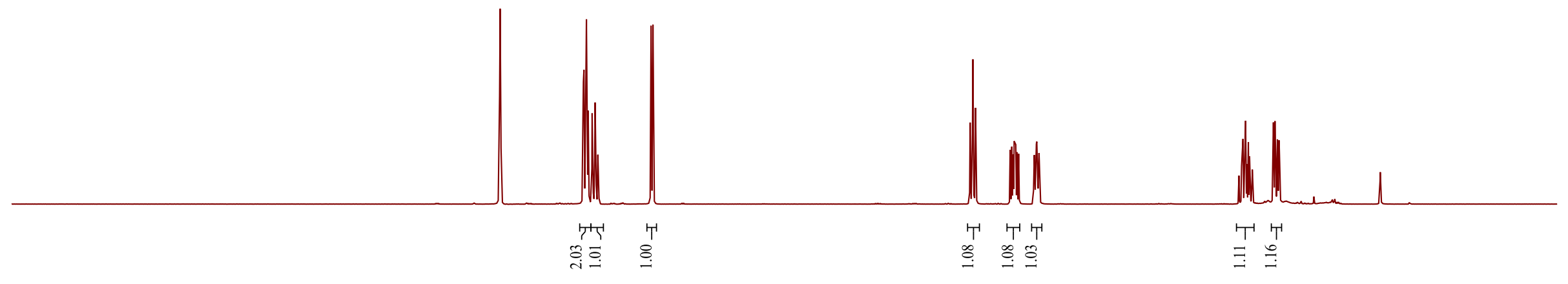

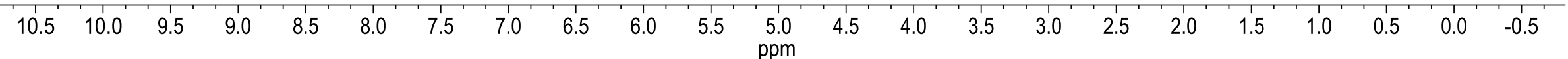




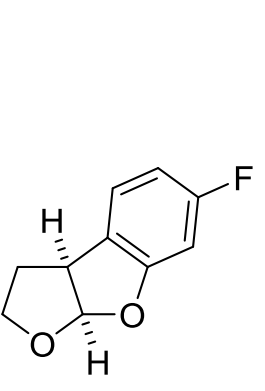

(3i)

${ }^{13} \mathrm{C}\left\{{ }^{1} \mathrm{H}\right\}-N M R$

$\mathrm{C}_{6} \mathrm{D}_{6}, 100 \mathrm{MHz}, 298 \mathrm{~K}$

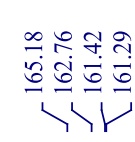

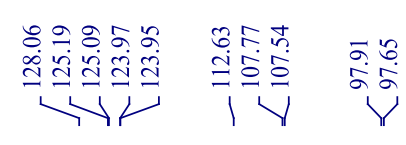

เి

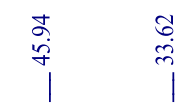

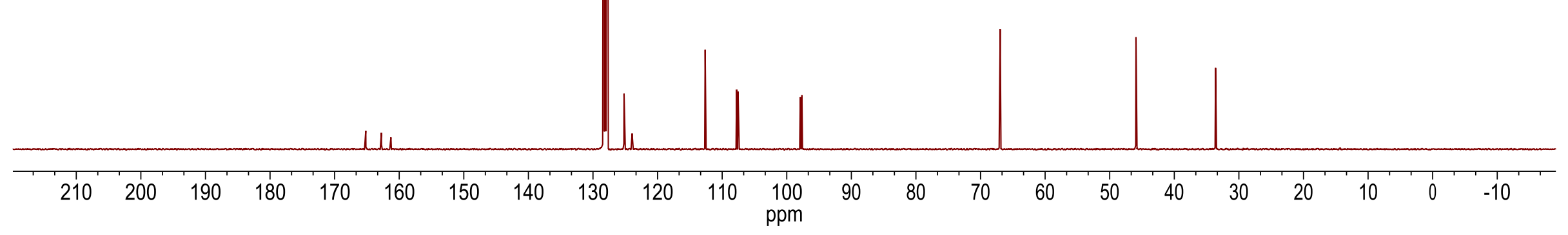




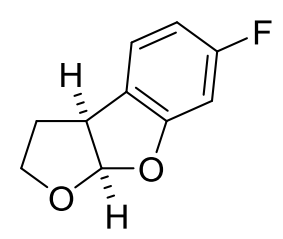

(3i)

${ }^{19} \mathrm{~F}-\mathrm{NMR}$

$\mathrm{C}_{6} \mathrm{D}_{6}, 282 \mathrm{MHz}, 298 \mathrm{~K}$

$\begin{array}{llllllllllllllllllllllllllllllll}0 & 10 & 0 & -10 & -20 & -30 & -40 & -50 & -60 & -70 & -80 & -90 & -100 & -110 & -120 & -130 & -140 & -150 & -160 & -170 & -180 & -190 & -200 & -210 & 1\end{array}$ 


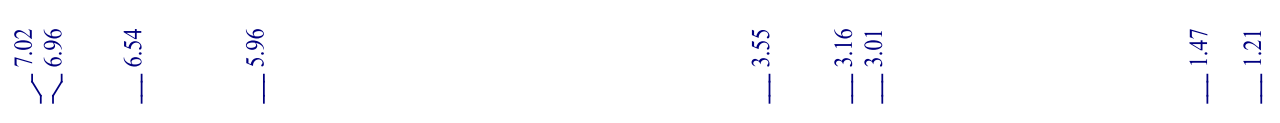

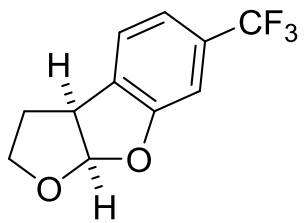

(3j)

${ }^{1} \mathrm{H}-\mathrm{NMR}$

$\mathrm{C}_{6} \mathrm{D}_{6}, 400 \mathrm{MHz}, 298 \mathrm{~K}$

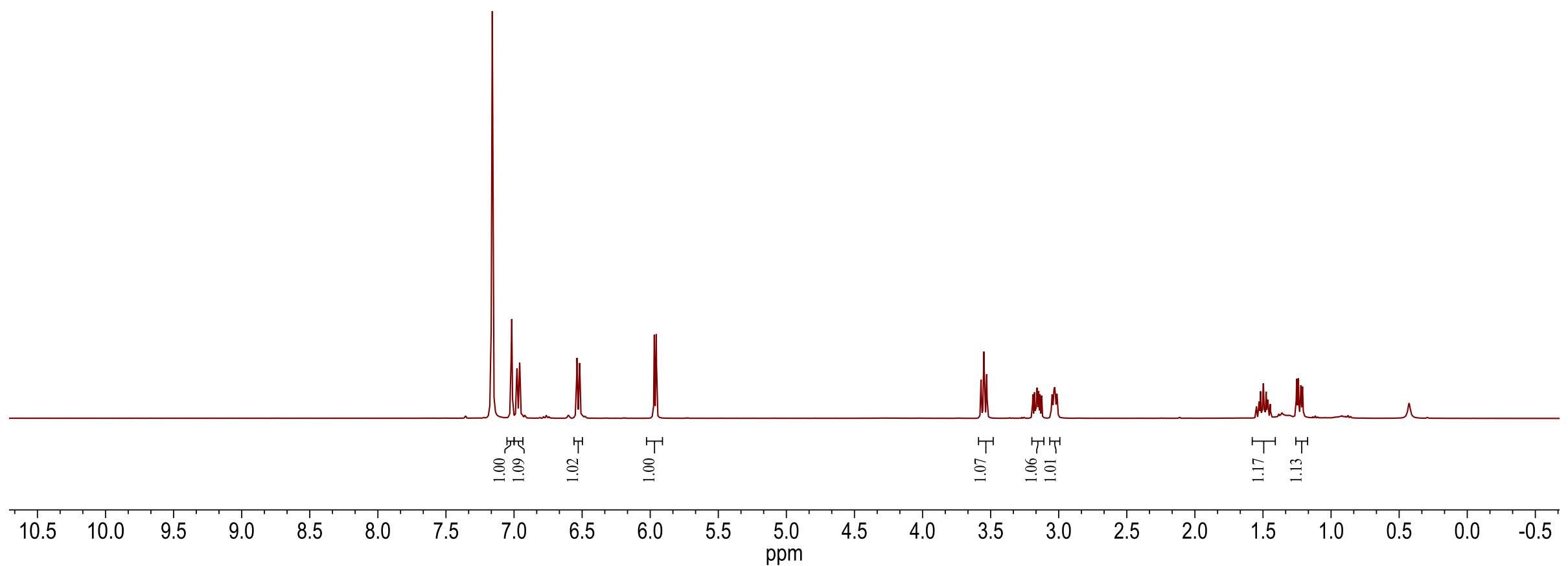




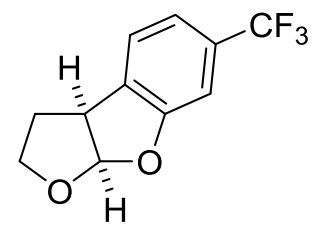

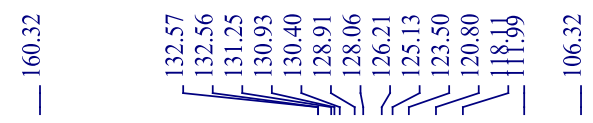

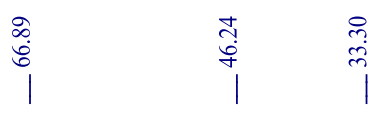

(3j)

${ }^{13} \mathrm{C}\left\{{ }^{1} \mathrm{H}\right\}-\mathrm{NMR}$

$\mathrm{C}_{6} \mathrm{D}_{6}, 100 \mathrm{MHz}, 298 \mathrm{~K}$

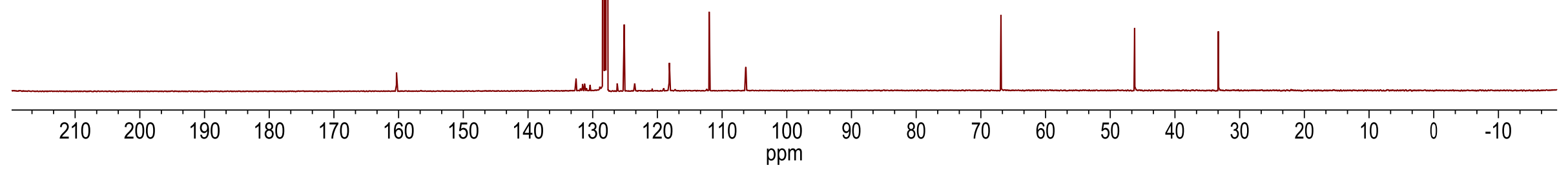




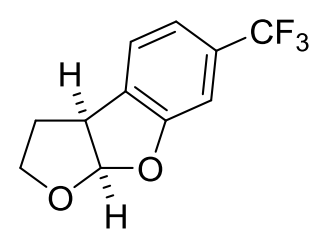

\section{$\underset{\substack{0 \\ i}}{i}$}

(3j)

${ }^{19} \mathrm{~F}-\mathrm{NMR}$

$\mathrm{C}_{6} \mathrm{D}_{6}, 282 \mathrm{MHz}, 298 \mathrm{~K}$

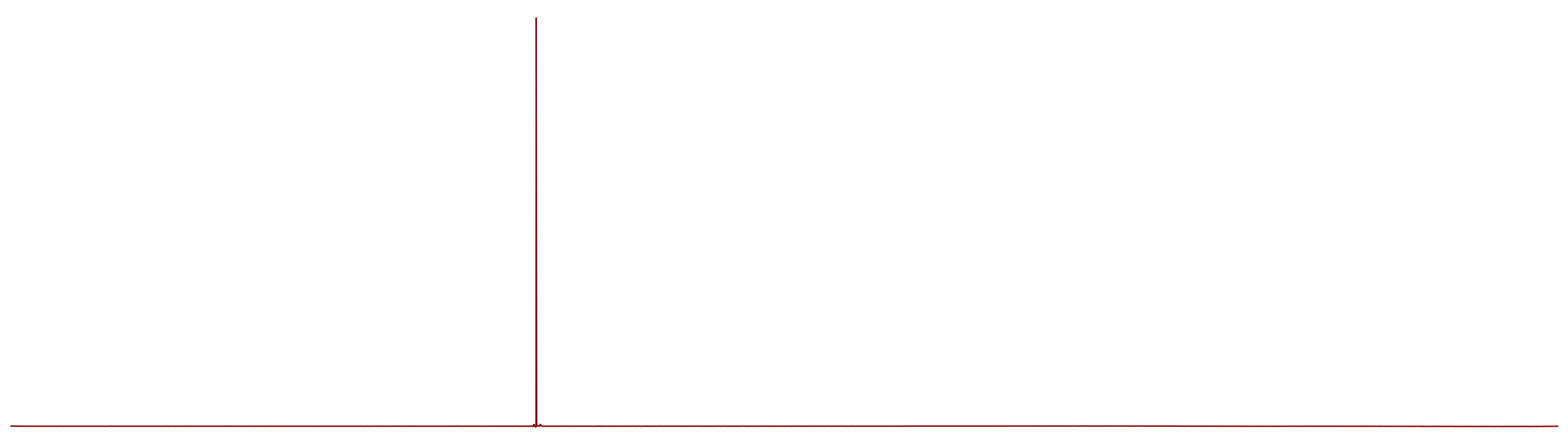

$\begin{array}{llllllllllllllllllllllllllllll}10 & 0 & -10 & -20 & -30 & -40 & -50 & -60 & -70 & -80 & -90 & -100 & -110 & -120 & -130 & -140 & -150 & -160 & -170 & -180 & -190 & -200 & -210 & \end{array}$




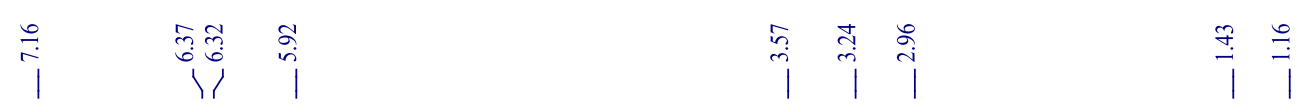

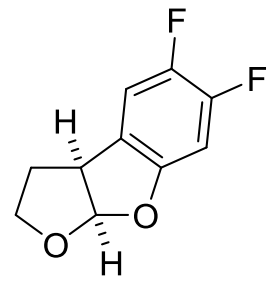

(3k)

${ }^{1} \mathrm{H}-\mathrm{NMR}$

$\mathrm{C}_{6} \mathrm{D}_{6}, 400 \mathrm{MHz}, 298 \mathrm{~K}$

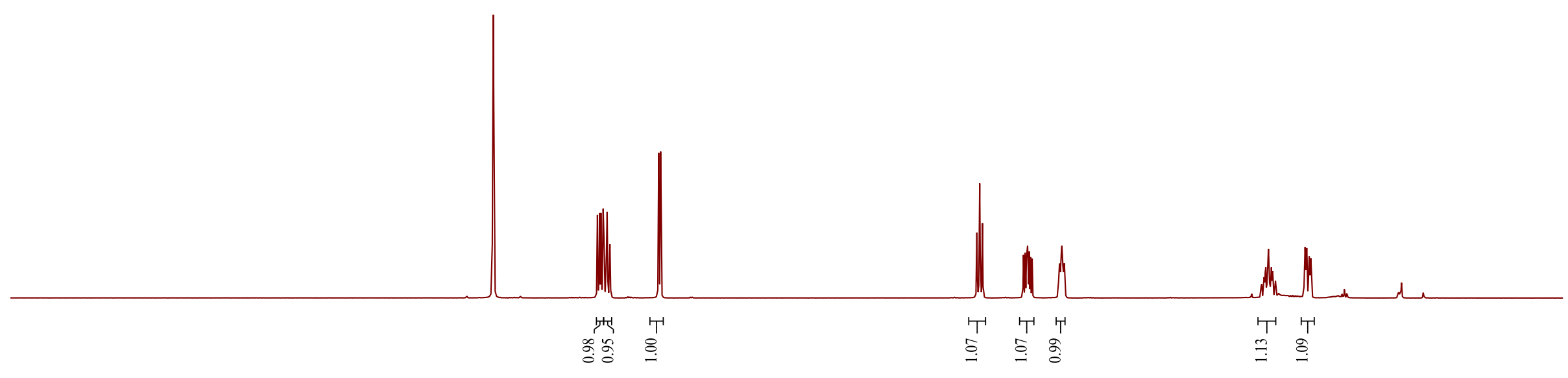

\begin{tabular}{lllllllllllllllllllllllll}
\hline 10.5 & 10.0 & 9.5 & 9.0 & 8.5 & 8.0 & 7.5 & 7.0 & 6.5 & 6.0 & 5.5 & 5.0 & 4.5 & 4.0 & 3.5 & 3.0 & 2.5 & 2.0 & 1.5 & 1.0 & 0.5 & 0.0 & -0.5
\end{tabular}




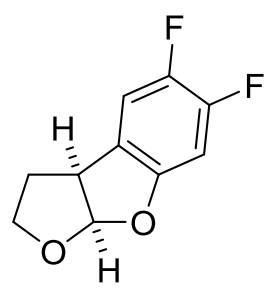

(3k)

${ }^{13} \mathrm{C}\left\{{ }^{1} \mathrm{H}\right\}-\mathrm{NMR}$

$\mathrm{C}_{6} \mathrm{D}_{6}, 100 \mathrm{MHz}, 298 \mathrm{~K}$
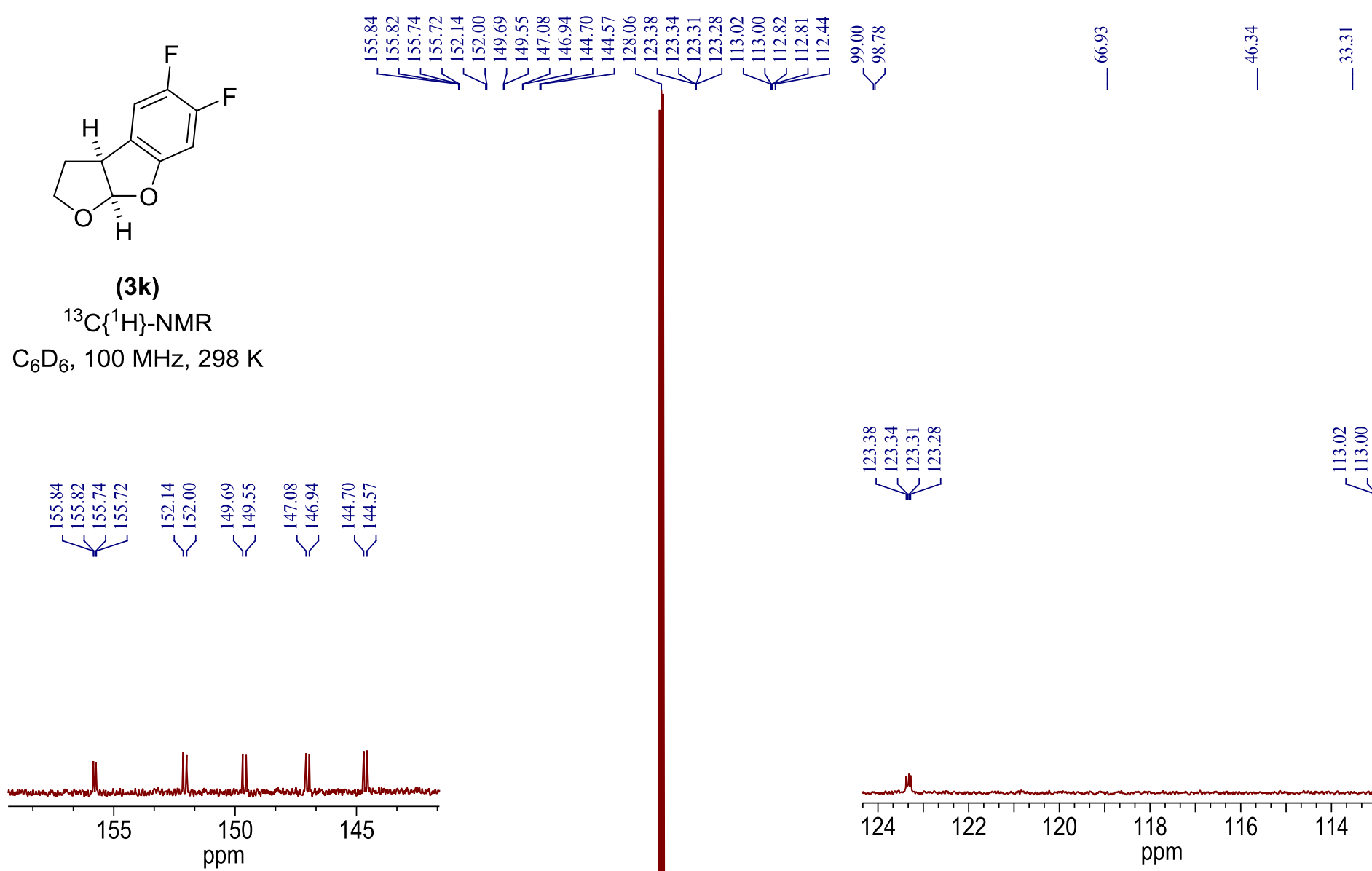

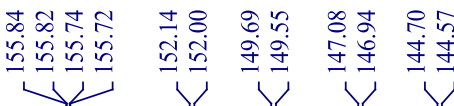
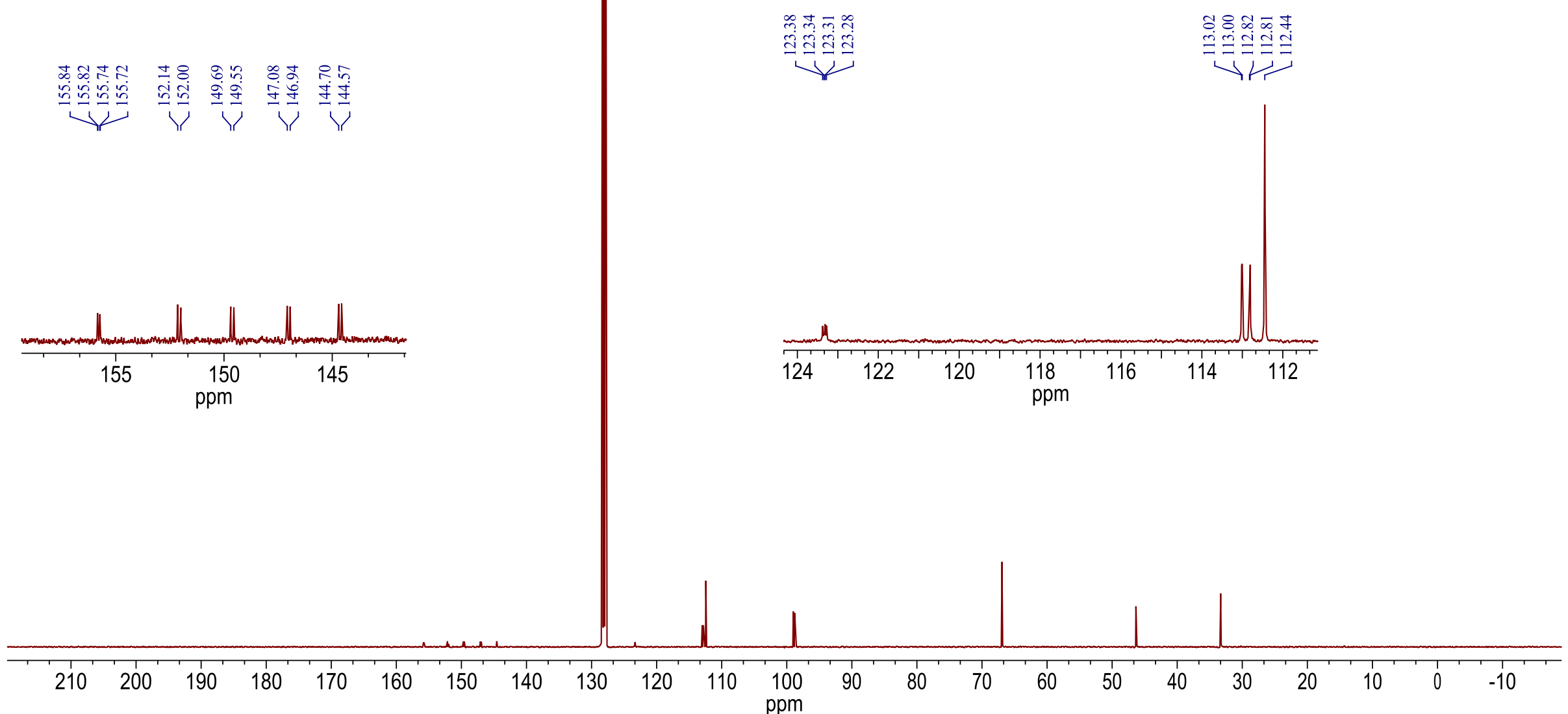

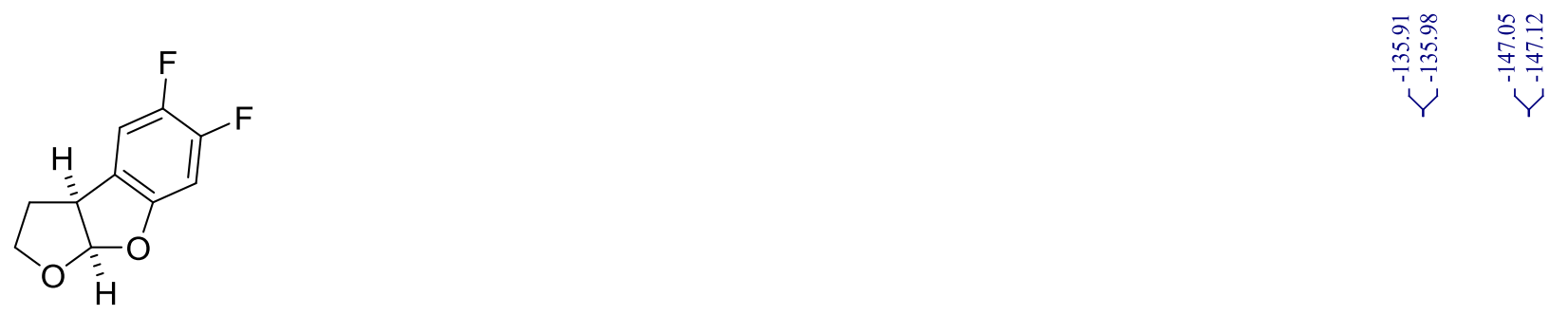

(3k)

${ }^{19} \mathrm{~F}-\mathrm{NMR}$

$\mathrm{C}_{6} \mathrm{D}_{6}, 282 \mathrm{MHz}, 298 \mathrm{~K}$

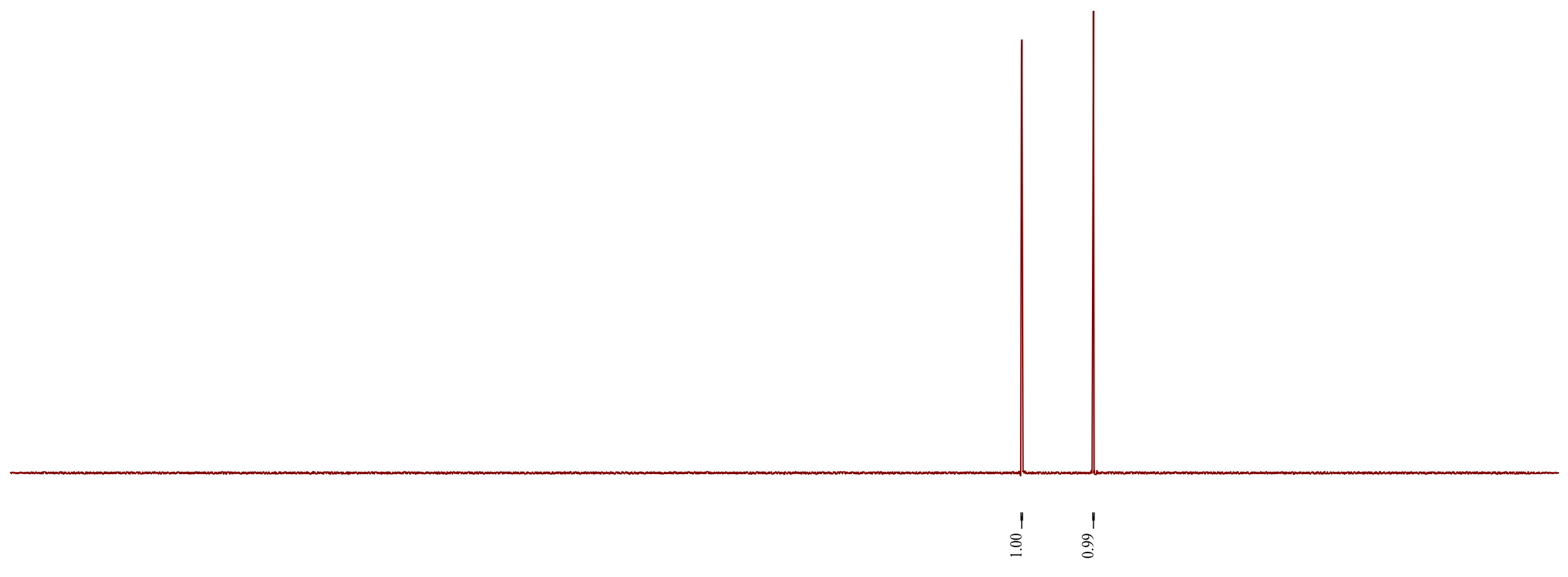

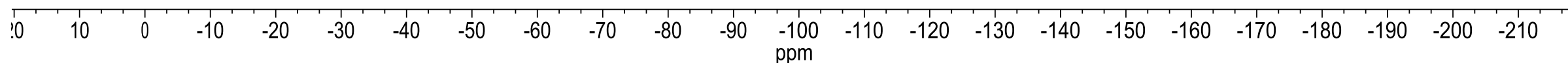




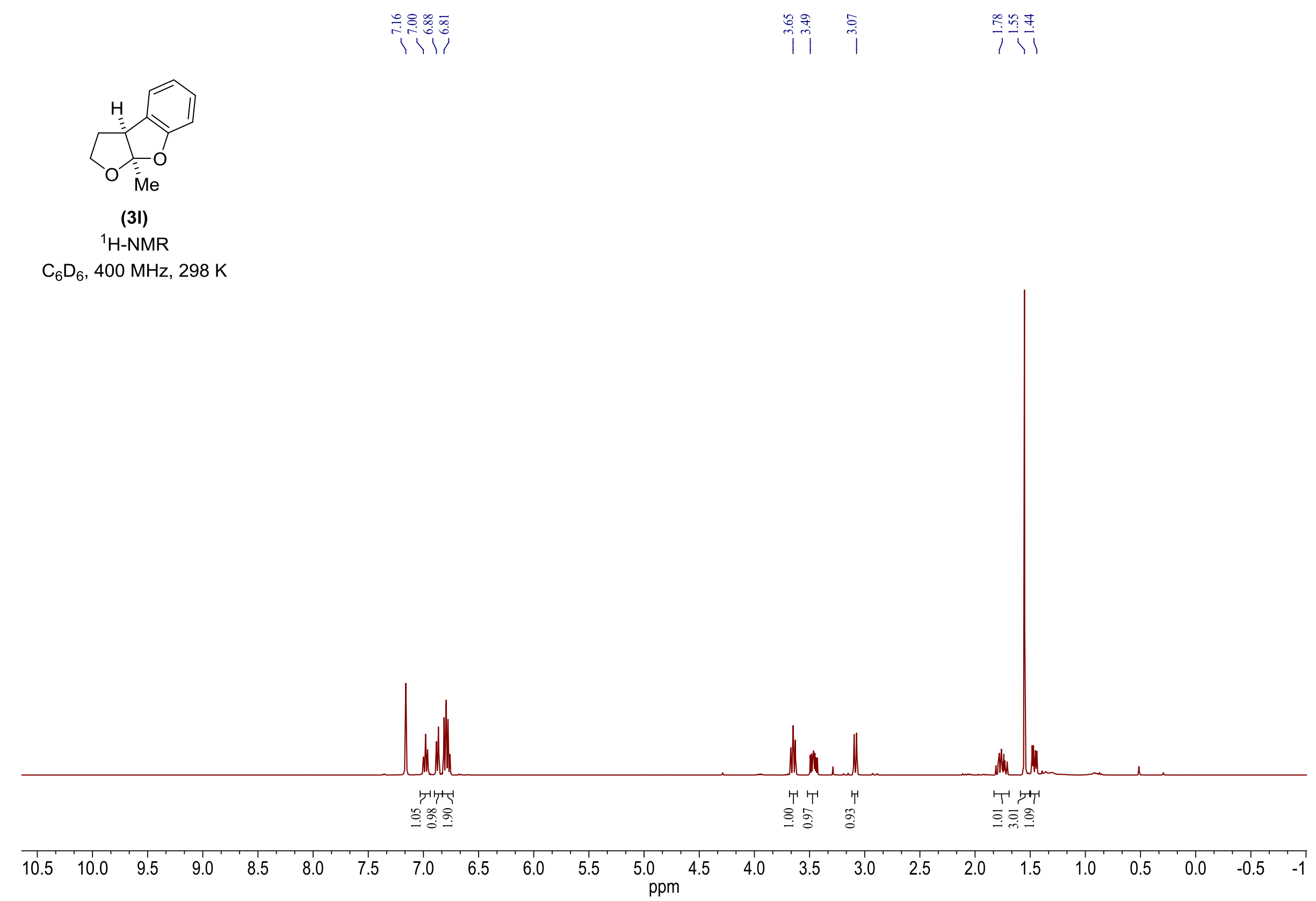




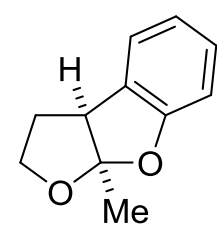

(3I)

${ }^{13} \mathrm{C}\left\{{ }^{1} \mathrm{H}\right\}-\mathrm{NMR}$

$\mathrm{C}_{6} \mathrm{D}_{6}, 100 \mathrm{MHz}, 298 \mathrm{~K}$

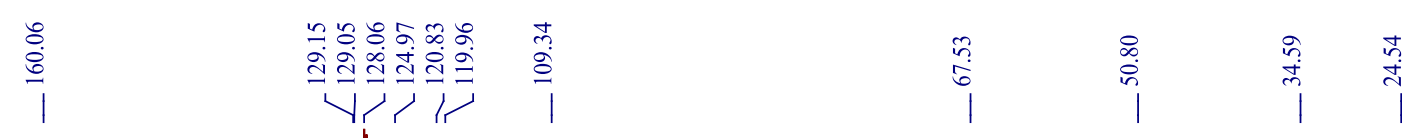

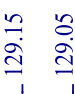

|

129.2129 .0

ppm

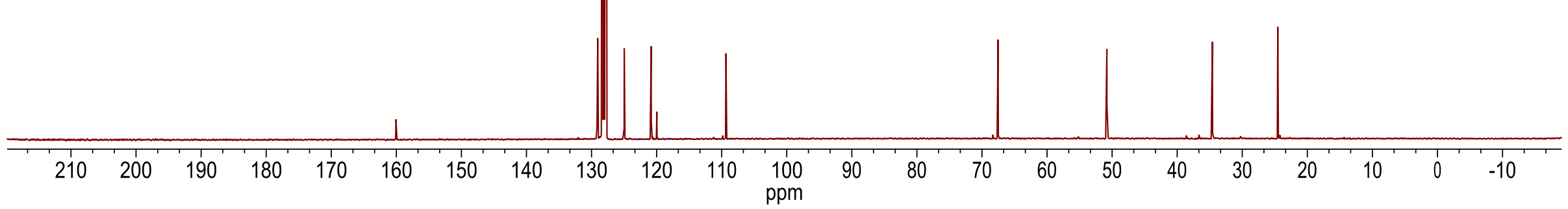




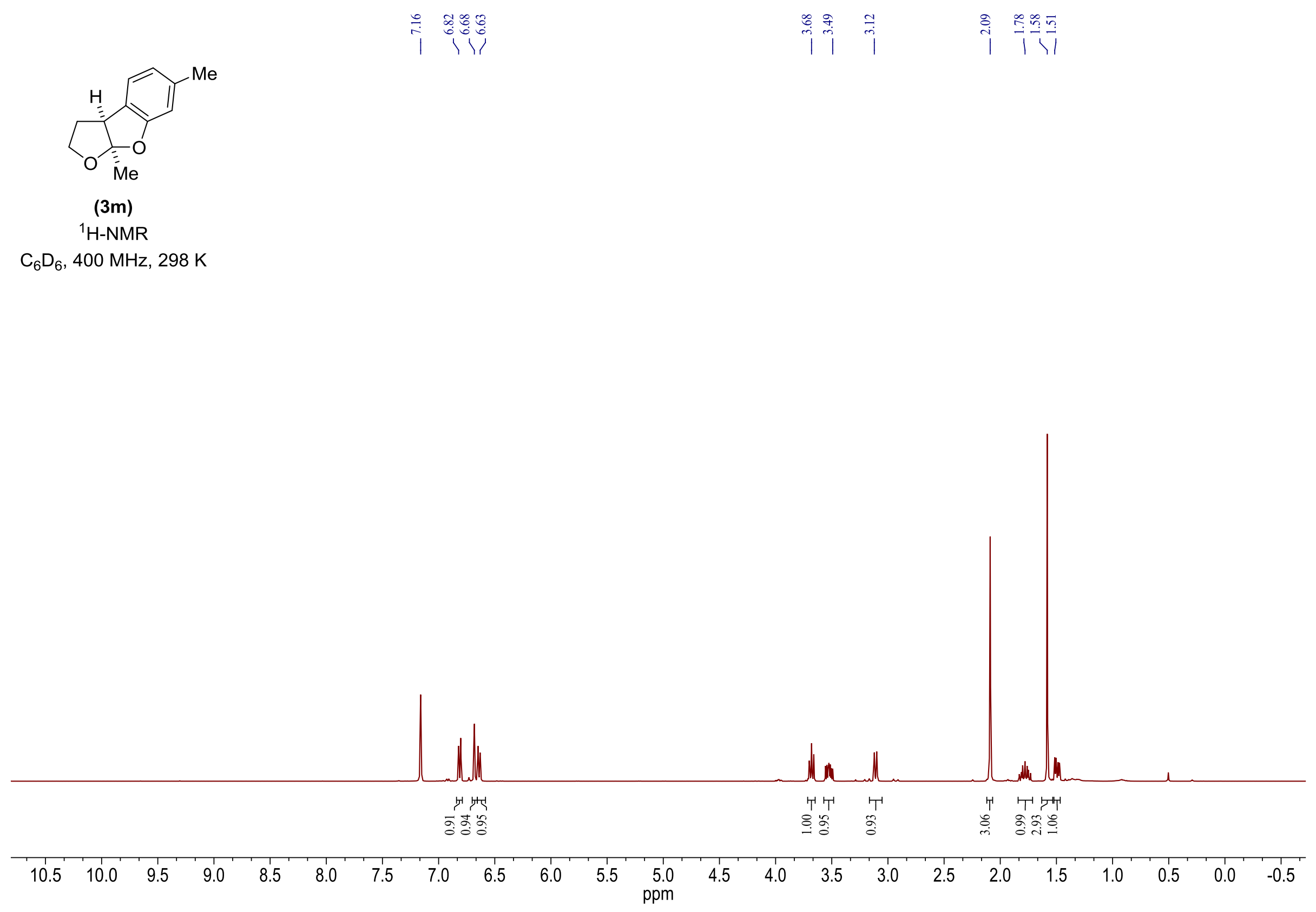




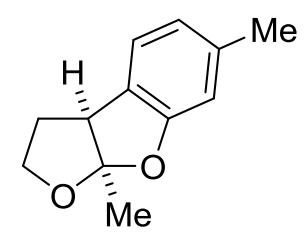

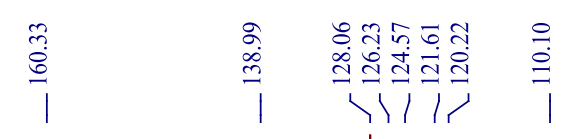

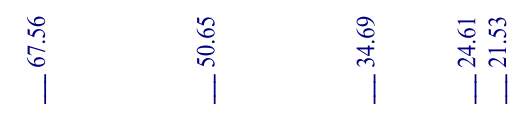

(3m)

${ }^{13} \mathrm{C}\left\{{ }^{1} \mathrm{H}\right\}-\mathrm{NMR}$

$\mathrm{C}_{6} \mathrm{D}_{6}, 100 \mathrm{MHz}, 298 \mathrm{~K}$

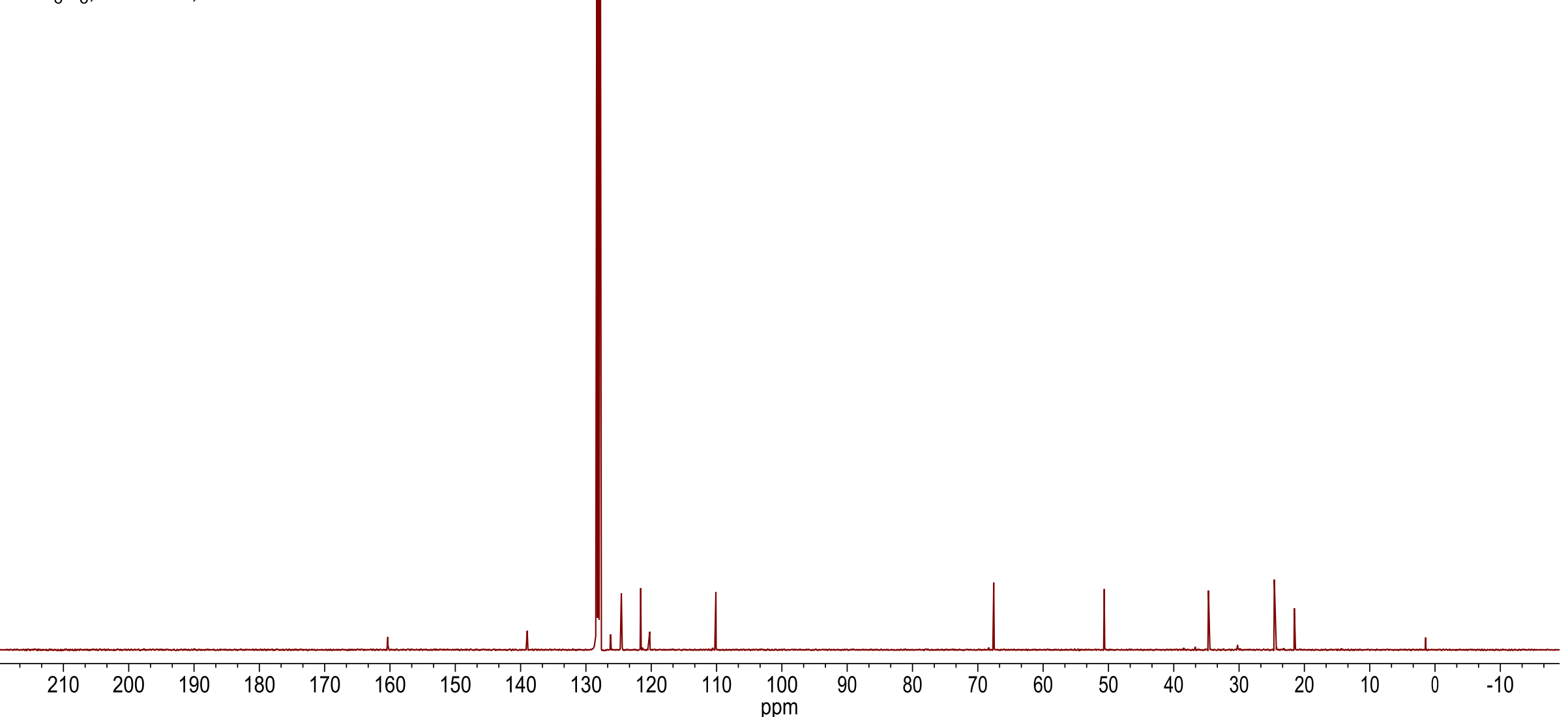




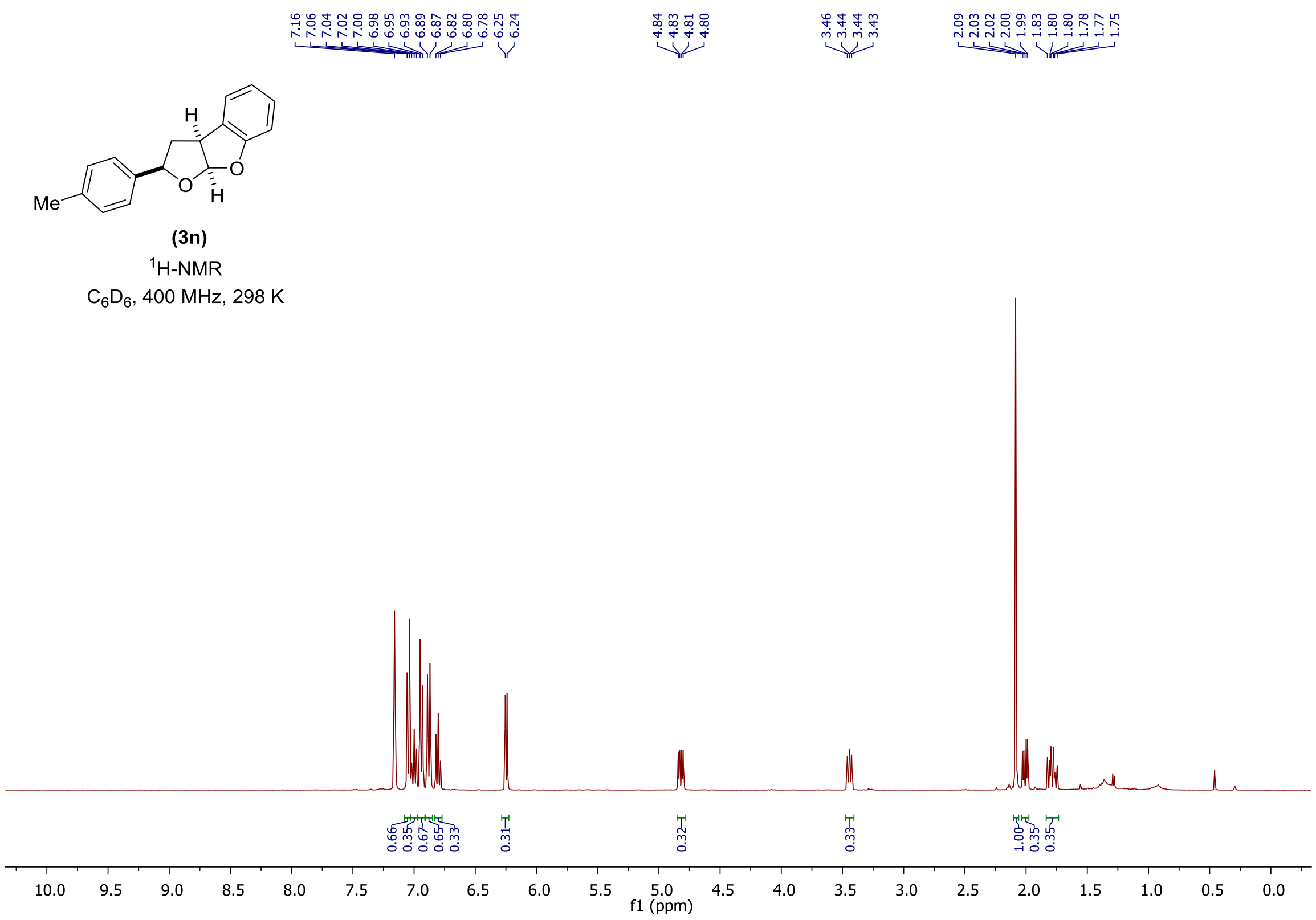




|

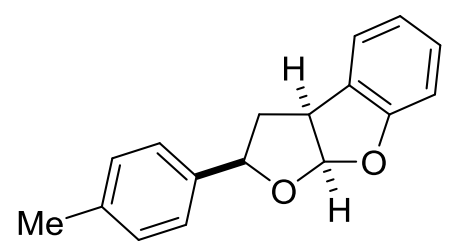

(3n)

${ }^{13} \mathrm{C}\left\{{ }^{1} \mathrm{H}\right\}-\mathrm{NMR}$

$\mathrm{C}_{6} \mathrm{D}_{6}, 100 \mathrm{MHz}, 298 \mathrm{~K}$

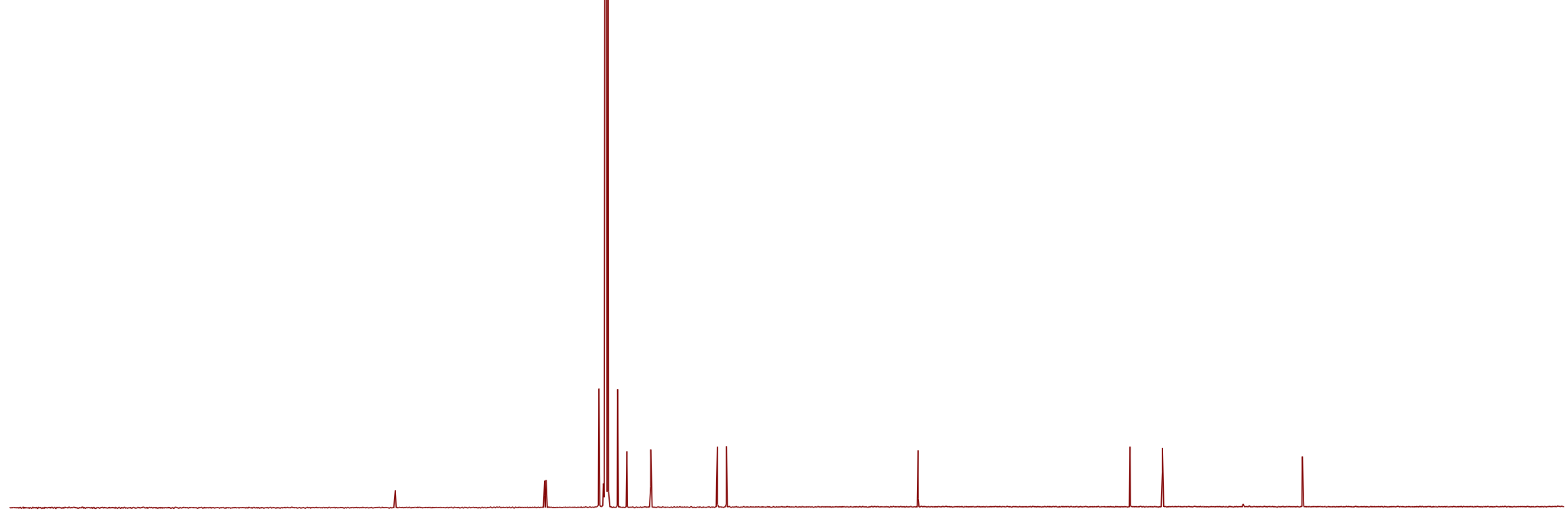

$\begin{array}{llllllllllllllllllllllll}210 & 200 & 190 & 180 & 170 & 160 & 150 & 140 & 130 & 120 & 110 & 100 & 90 & 80 & 70 & 60 & 50 & 40 & 30 & 20 & 10 & 0 & -10\end{array}$ 


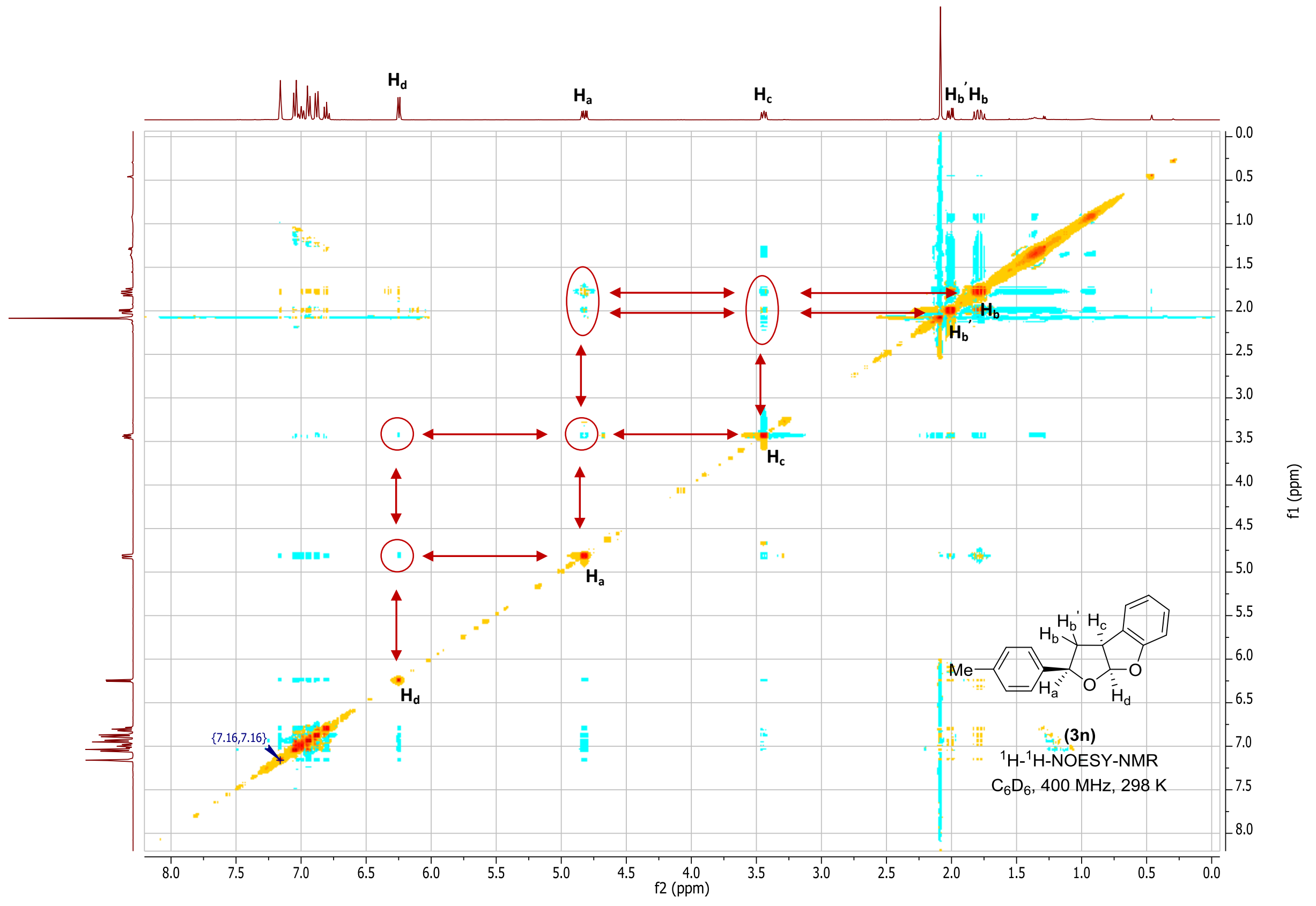




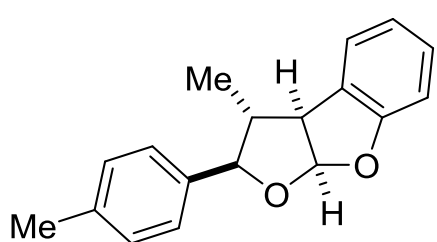

(30)

${ }^{1} \mathrm{H}-\mathrm{NMR}$

$\mathrm{C}_{6} \mathrm{D}_{6}, 400 \mathrm{MHz}, 298 \mathrm{~K}$
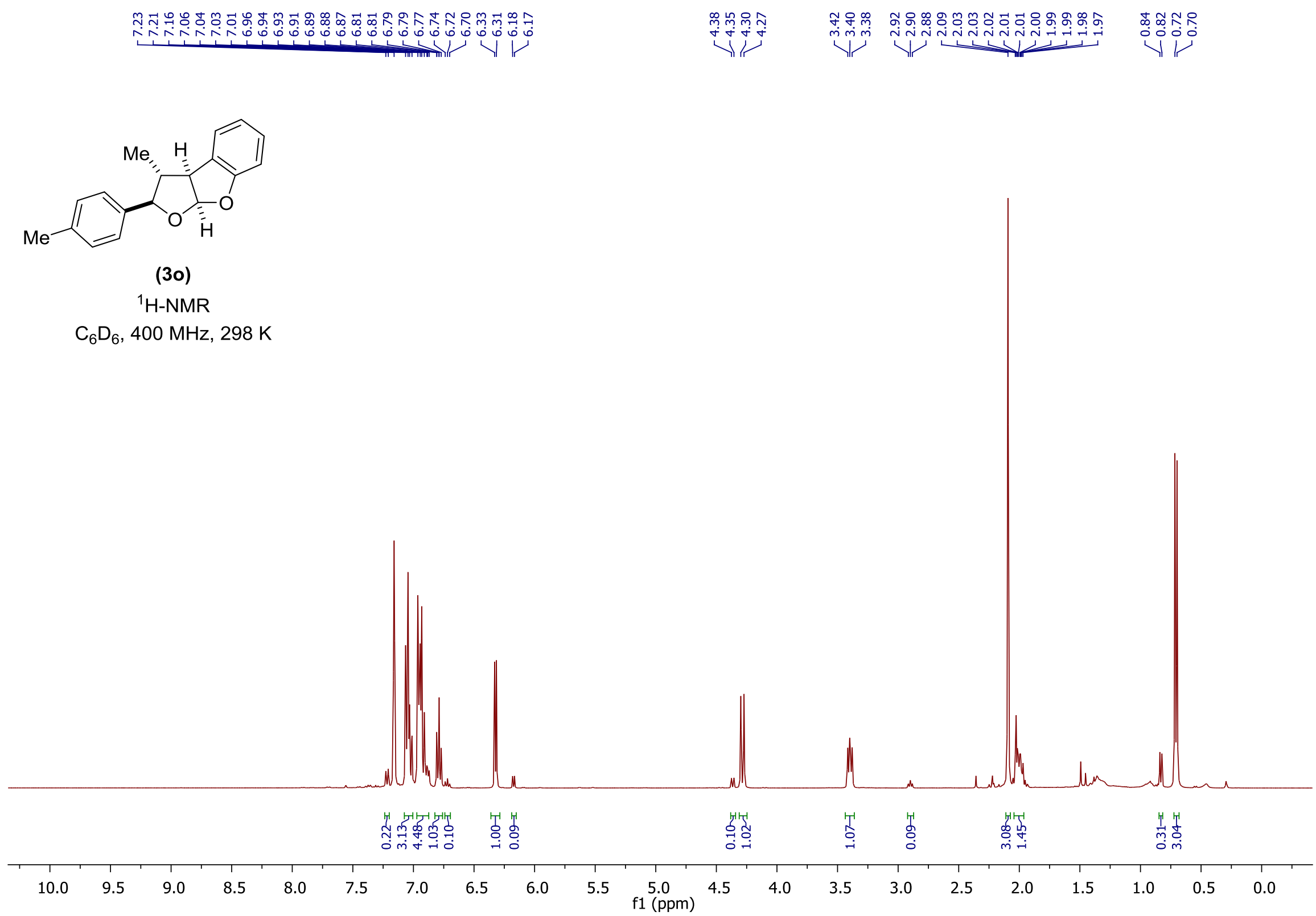

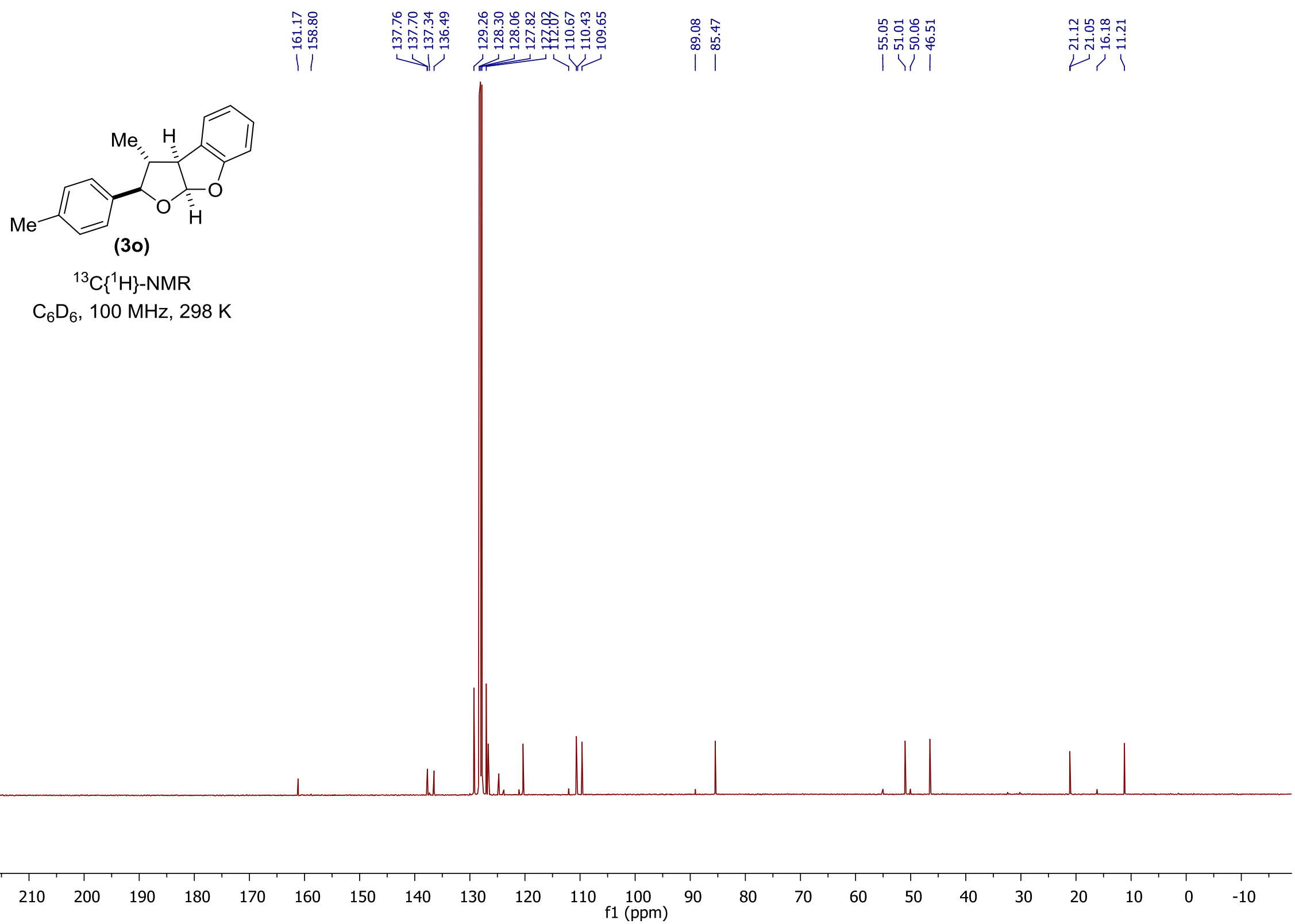


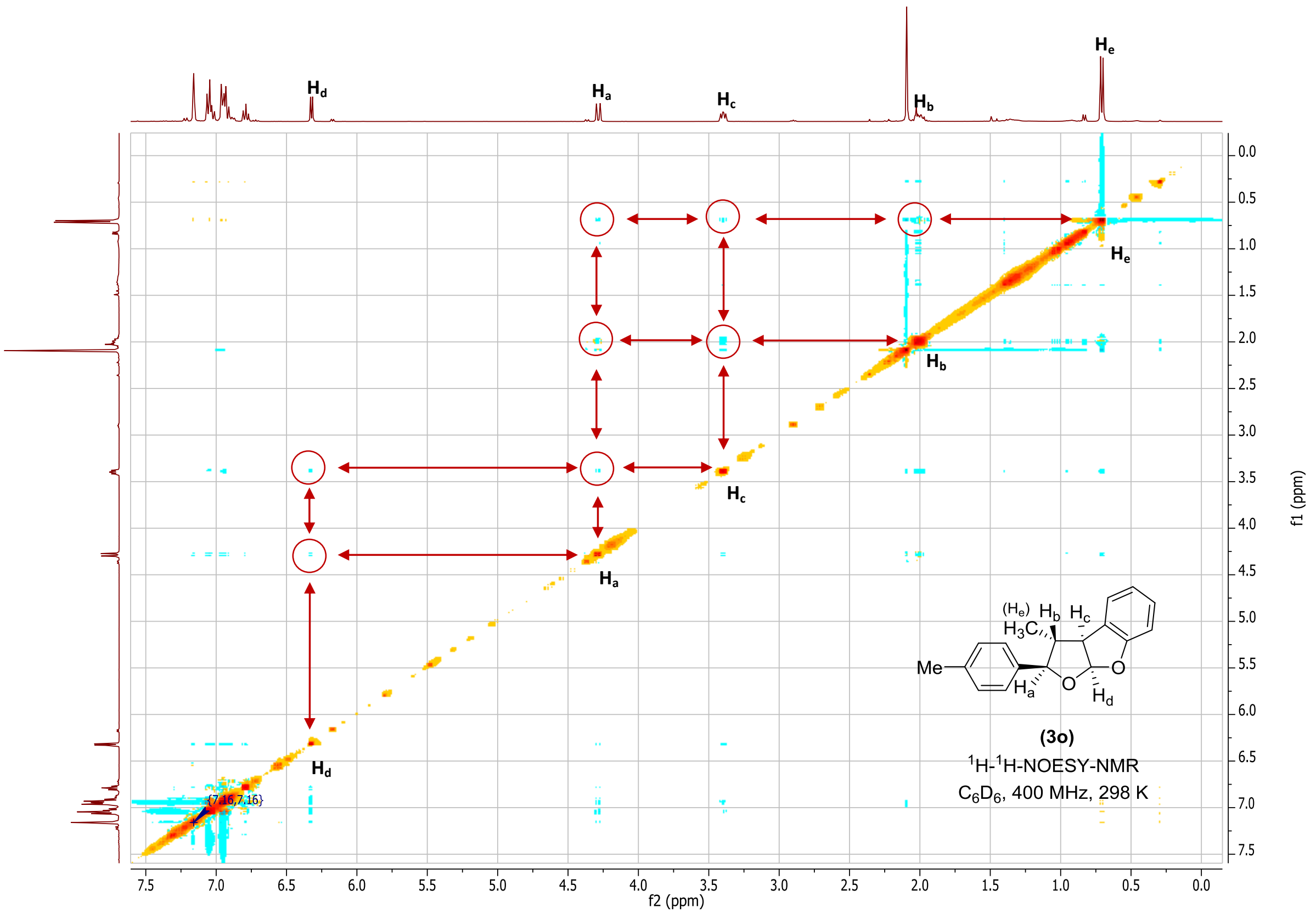




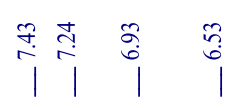
$\stackrel{\substack{n \\ \infty}}{\stackrel{n}{\infty}}$

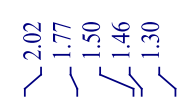<smiles>Brc1ccccc1OC1CCCO1</smiles>

(4a)

${ }^{1} \mathrm{H}-\mathrm{NMR}$

$\mathrm{C}_{6} \mathrm{D}_{6}, 400 \mathrm{MHz}, 298 \mathrm{~K}$

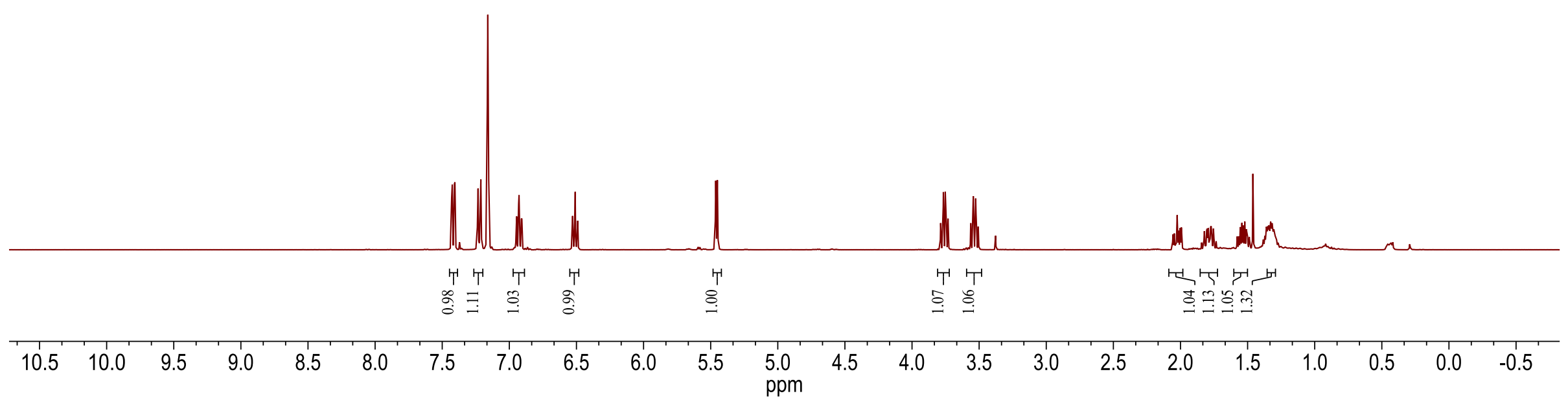




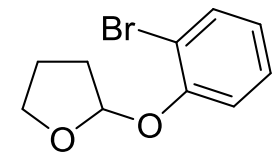

(4a)

${ }^{13} \mathrm{C}\left\{{ }^{1} \mathrm{H}\right\}-\mathrm{NMR}$

$\mathrm{CDCl}_{3}, 100 \mathrm{MHz}, 298 \mathrm{~K}$

$\begin{array}{lll}0 & 1 \\ 0 & 1\end{array}$

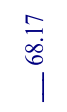

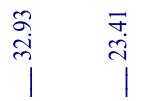

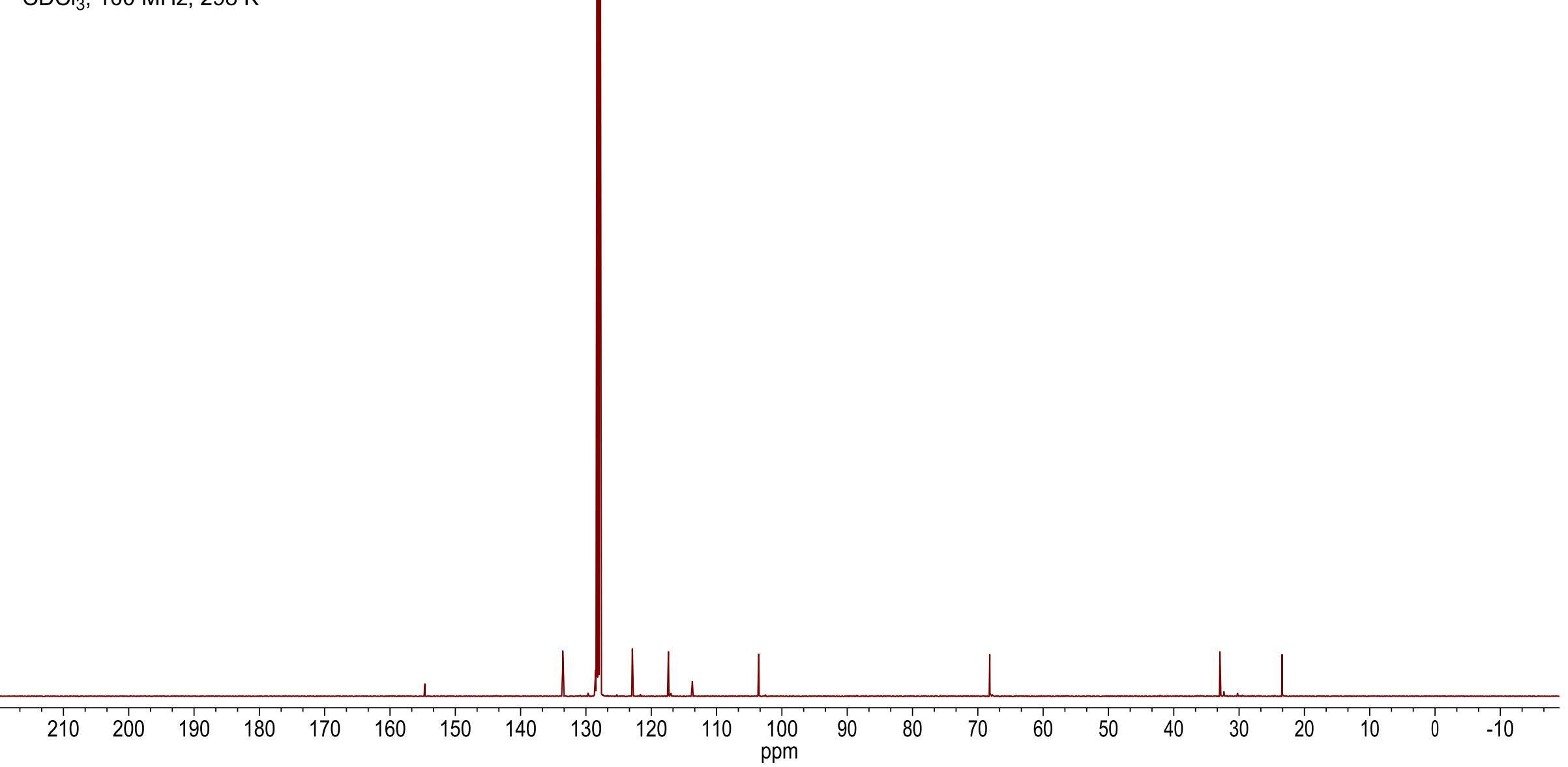




\section{$6 \mathrm{X}$-ray structure analyses}

\section{- X-ray structure determination}

Details of the structure refinement are given in Table 1. Displacement ellipsoid representations are depicted in Figure 1.

\section{Table 1 Crystal data and structure refinement}

\begin{tabular}{|c|c|c|c|}
\hline & $3 c$ & $3 h^{*}$ & 3o (minor diastereoisomer) \\
\hline CCDC number & 1455967 & 1455966 & 1455965 \\
\hline Empirical formula & $\mathrm{C}_{11} \mathrm{H}_{12} \mathrm{O}_{2}$ & $\mathrm{C}_{10} \mathrm{H}_{8} \mathrm{~F}_{2} \mathrm{O}_{2}$ & $\mathrm{C}_{18} \mathrm{H}_{18} \mathrm{O}_{2}$ \\
\hline Formula weight & 176.21 & 198.16 & 266.32 \\
\hline Temperature/K & $181(2)$ & $180.1(2)$ & $180.00(14)$ \\
\hline Crystal system & orthorhombic & triclinic & orthorhombic \\
\hline Space group & $\mathrm{P} 22_{2}{ }_{1}{ }_{1}$ & P1 & $\mathrm{P} 22_{1} 2_{1}$ \\
\hline $\mathrm{a} / \AA ̊ A$ & $4.7621(2)$ & $5.4814(12)$ & $7.43203(5)$ \\
\hline $\mathrm{b} / \mathrm{A}$ & $8.4156(4)$ & $8.5363(17)$ & $8.92309(5)$ \\
\hline$c / A ̊$ & $22.4052(12)$ & $10.1397(17)$ & $21.25373(13)$ \\
\hline$\alpha /^{\circ}$ & 90 & $71.243(16)$ & 90 \\
\hline$\beta /{ }^{\circ}$ & 90 & $75.321(16)$ & 90 \\
\hline $\mathrm{y}^{\circ}$ & 90 & $76.523(18)$ & 90 \\
\hline Volume $/ \AA^{3}$ & 897.91(8) & $428.59(16)$ & $1409.478(15)$ \\
\hline Z & 4 & 2 & 4 \\
\hline$\rho_{\text {calc }} \mathrm{g} / \mathrm{cm}^{3}$ & 1.303 & 1.536 & 1.255 \\
\hline$\mu / \mathrm{mm}^{-1}$ & 0.715 & 1.170 & 0.635 \\
\hline$F(000)$ & 376.0 & 204.0 & 568.0 \\
\hline Crystal size $/ \mathrm{mm}^{3}$ & $0.6694 \times 0.2407 \times 0.0855$ & $0.6143 \times 0.2488 \times 0.1202$ & $0.3332 \times 0.278 \times 0.1017$ \\
\hline Radiation & CuKa $(\lambda=1.5418)$ & CuKa $(\lambda=1.5418)$ & CuKa $(\lambda=1.5418)$ \\
\hline $2 \theta$ range for data collection ${ }^{\circ}$ & 7.892 to 145.792 & 9.39 to 152.836 & 8.32 to 146.842 \\
\hline Index ranges & $\begin{array}{l}-5 \leq h \leq 5 \\
-8 \leq k \leq 10 \\
-27 \leq 1 \leq 27\end{array}$ & $\begin{array}{l}-6 \leq h \leq 6 \\
-10 \leq k \leq 10 \\
-12 \leq 1 \leq 12\end{array}$ & $\begin{array}{l}-9 \leq h \leq 9, \\
-11 \leq k \leq 11, \\
-26 \leq 1 \leq 26\end{array}$ \\
\hline Reflections collected & 5208 & 3423 & 48986 \\
\hline Independent reflections & $\begin{array}{l}1770 \\
R_{\text {int }} 0.0377, R_{\text {sigma }} 0.0298\end{array}$ & $\begin{array}{l}3423 \\
\mathrm{R}_{\text {int }}\end{array}$ & $\begin{array}{l}2831 \\
R_{\text {int }} 0.0304, R_{\text {sigma }} 0.0099\end{array}$ \\
\hline Data/restraints/parameters & $1770 / 0 / 120$ & $3423 / 3 / 254$ & $2831 / 0 / 185$ \\
\hline Goodness-of-fit on $\mathrm{F}^{2}$ & 1.069 & 1.074 & 1.079 \\
\hline Final $R$ indexes $[I>=2 \sigma(I)]$ & $\mathrm{R}_{1}=0.0425, \mathrm{wR}_{2}=0.1172$ & $R_{1}=0.0432, w R_{2}=0.1188$ & $\mathrm{R}_{1}=0.0254, \mathrm{wR}_{2}=0.0639$ \\
\hline Final $\mathrm{R}$ indexes [all data] & $\mathrm{R}_{1}=0.0454, \mathrm{wR}_{2}=0.1200$ & $\mathrm{R}_{1}=0.0440, \mathrm{wR}_{2}=0.1205$ & $\mathrm{R}_{1}=0.0263, \mathrm{wR}_{2}=0.0646$ \\
\hline Largest diff. peak/hole / e $\AA^{-3}$ & $0.19 /-0.21$ & $0.23 /-0.18$ & $0.15 /-0.10$ \\
\hline
\end{tabular}

*For $3 h$, the crystal was twinned by a two-fold rotation along the a-axis. The two individual domains were integrated and the final refinement was carried out on the combined reflection file (HKLF5 file). Table 1 show the crystallographic data for the twinned refinement. 
Figure 1: Displacement ellipsoid representation of the structure (50 percent probability). For $3 h$, there are two independent molecules in the asymmetric unit that are superimposable.

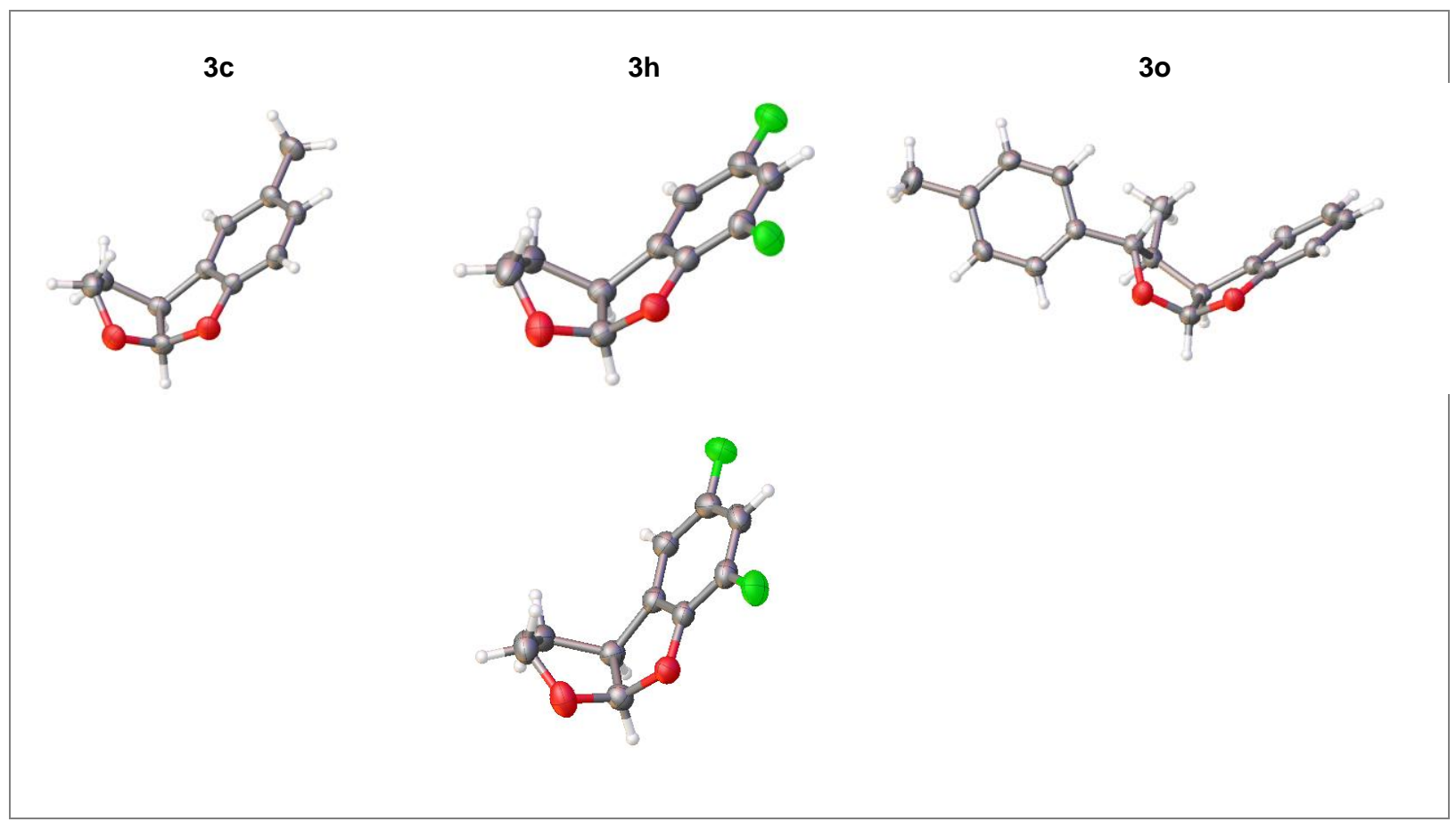

- Absolute structure determination

It is now well established that for good-quality data absolute configuration can be determined using X-ray diffraction with $\mathrm{Cu}$ radiation even for light atoms structures ${ }^{6,7}$. Analysis of the Bijvoet differences was carried out using the Platon software ${ }^{8}$.

For structure 30, the crystal quality is good. The Friedif is of 27. A full-sphere highly redundant dataset was collected. Very good confidence in the absolute structure determination can be gained from the Bayesian analysis of the Bijvoet differences. Using a Gaussian distribution, the normal probability plot for the data has a slope of 0.962 with a correlation coefficient of 0.996 . The Hooft parameter was calculated as $-0.04(4)$. Using the t-probability plot the correlation becomes 0.999 and the slope 0.923 , with a calculated Hooft parameter of $-0.06(5)$.

The Flack parameter obtained by post-processing of the data using the Parsons' method is $-0.06(4)$ using 1133 Bijvoet pairs.

For structure $\mathbf{3 c}$, The Friedif is 31 . The data quality is slightly lower (the diffraction spot were not as nicely shaped). However, the analysis of Bijvoet differences also gives us confidence in the absolute structure determination. Using the Gaussian distribution, the normal probability plot for the data has a slope of 0.971 with a correlation coefficient of 0.971 . The Hooft parameter was calculated as $-0.03(15)$. Using the tprobability plot the correlation becomes 0.997 and the slope 0.921 , with a calculated Hooft parameter of $0.11(15)$.

The flack parameter obtained by post-processing of the data using the Parsons' method is $-0.19(15)$ using 621 bijvoet pairs. 
In the case of $\mathbf{3 h}$, non-merohedral twinning makes the assignment more complicated. However, the presence of the fluorine atoms makes the Bijvoet differences higher (Friedif of 54) and fortunately most of the reflections from the two individual twin domains were not overlapped. (Twin domain 1: 6644 separated and 996 overlapped reflection; twin domain 2: 6619 separated and 996 overlapped reflections). For each domain, a classical refinement using the non-overlapped reflections could therefore be carried out. Using these refinements, the absolute structure can also be confidently assigned, as shown in Table 2 .

Table 2: Crystal data and absolute structure analyses for individual twin components of $3 \mathrm{~h}$

\begin{tabular}{|c|c|c|}
\hline & TWIN COMPONENT 1 & TWIN COMPONENT 2 \\
\hline Rint & 0.034 & 0.036 \\
\hline Redundancy & 2.3 & 2.3 \\
\hline Mean F2/sig(F2) & 77.7 & 70.6 \\
\hline Final $R$ indexes $[\mid>=2 \sigma(I)]$ & $R_{1}=0.0316, w R_{2}=0.0872$ & $R_{1}=0.0342, w R_{2}=0.0918$ \\
\hline Final $R$ indexes [all data] & $\mathrm{R}_{1}=0.0321, w \mathrm{R}_{2}=0.0877$ & $\mathrm{R}_{1}=0.0348, \mathrm{wR}_{2}=0.0923$ \\
\hline Data/parameters & $2835 / 253$ & $2842 / 253$ \\
\hline completeness & $81 \%$ & $80 \%$ \\
\hline Friedel coverage & $71 \%$ & $71 \%$ \\
\hline Flack (classical) & $-0.03(13)$ & $-0.05(14)$ \\
\hline Flack (Parson's) ${ }^{9}$ & $-0.014(94)$ for 1235 quotients & $0.076(92)$ for 1235 quotients \\
\hline Hooft(student distribution) $)^{10}$ & $-0.04(4)$ & $0.06(4)$ \\
\hline
\end{tabular}

\section{References}

$1 \quad$ Hungerland, T.; Düfert, A.; Objartel, I; Stalke, D.; Tietze; L-F. Chem. Eur. J., 2012, 18, 32866

Hooft, R. W. W.; Straver, L. H.; Spek, A. L. J. Appl. Crystallogr. 2008, 41, 96.

2 Chen, Y-Z.; Peng, M-L.; Zhang, D.; Zhang, L-P.; Wu, L-Z.; Tung, C-H. Tetrahedron 2006, 62, 10688.

3 Boal, B-W.; Schammel, A-W.; Garg, N-K. Org. Lett. 2009, 11, 3458

$4 \quad$ Wiley, R-A.; Zenk, P-C. Synthesis 1984, 695

$5 \quad$ Hillers, S.; Reiser, O. Synlett. 1995, 153

6 Hooft, R. W. W.; Straver, L. H.; Spek, A. L. J. Appl. Crystallogr. 2008, 41, 96.

7 Parsons, S.; Pattison, P.; Flack, H. D. Acta Crystallogr. A 2012, 68, 736.

8 Spek, A. L. J. Appl. Crystallogr. 2003, 36, 7.

9 Parsons, S.; Flack, H. D. ; Wagner, T. Acta Crystallogr. Sect. B Struct. Sci. Cryst. Eng. Mater. 2013, 69, 249.

10 Hooft, R. W. W.; Straver, L. H.; Spek, A. L.J. Appl. Crystallogr. 2010, 43, 665. 